Supplementary Information

for

\title{
Molybdenum Imido Alkylidene Complexes that Contain a $\beta$-Diketiminate Ligand
}

by

Zachary J. Tonzetich, Annie J. Jiang, Richard R. Schrock,* and Peter Müller

email: rrs@mit.edu

1. Variable temperature ${ }^{1} \mathrm{H}$ NMR spectra for $\mathbf{2 a}, \mathbf{2 d}$, and $\mathbf{4 a}$.

2. Kinetic plots for the thermal decomposition of $\mathbf{3 a}$ and $\mathbf{4 a}$.

3. Fully labeled thermal ellipsoid diagrams, crystal data and structure refinement parameters, atomic coordinates and equivalent isotropic displacement parameters, bond lengths and angles, and anisotropic displacement parameters for 1a, 3a", and $\mathbf{4 a}$. 


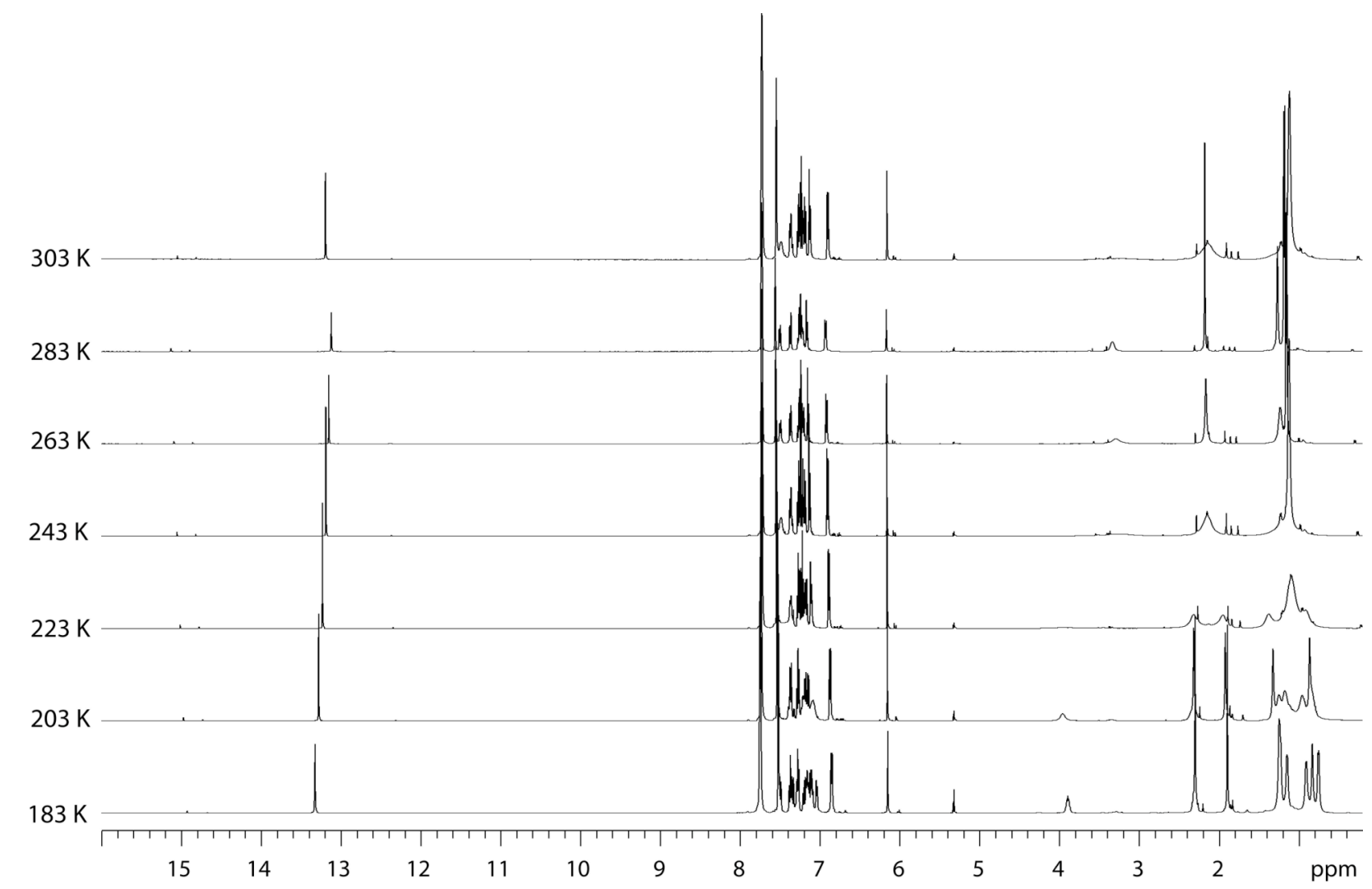

$500 \mathrm{MHz}{ }^{1} \mathrm{H}$ NMR variable temperature spectra of $\mathbf{2 a}$ in methylene chloride- $d_{2}$.

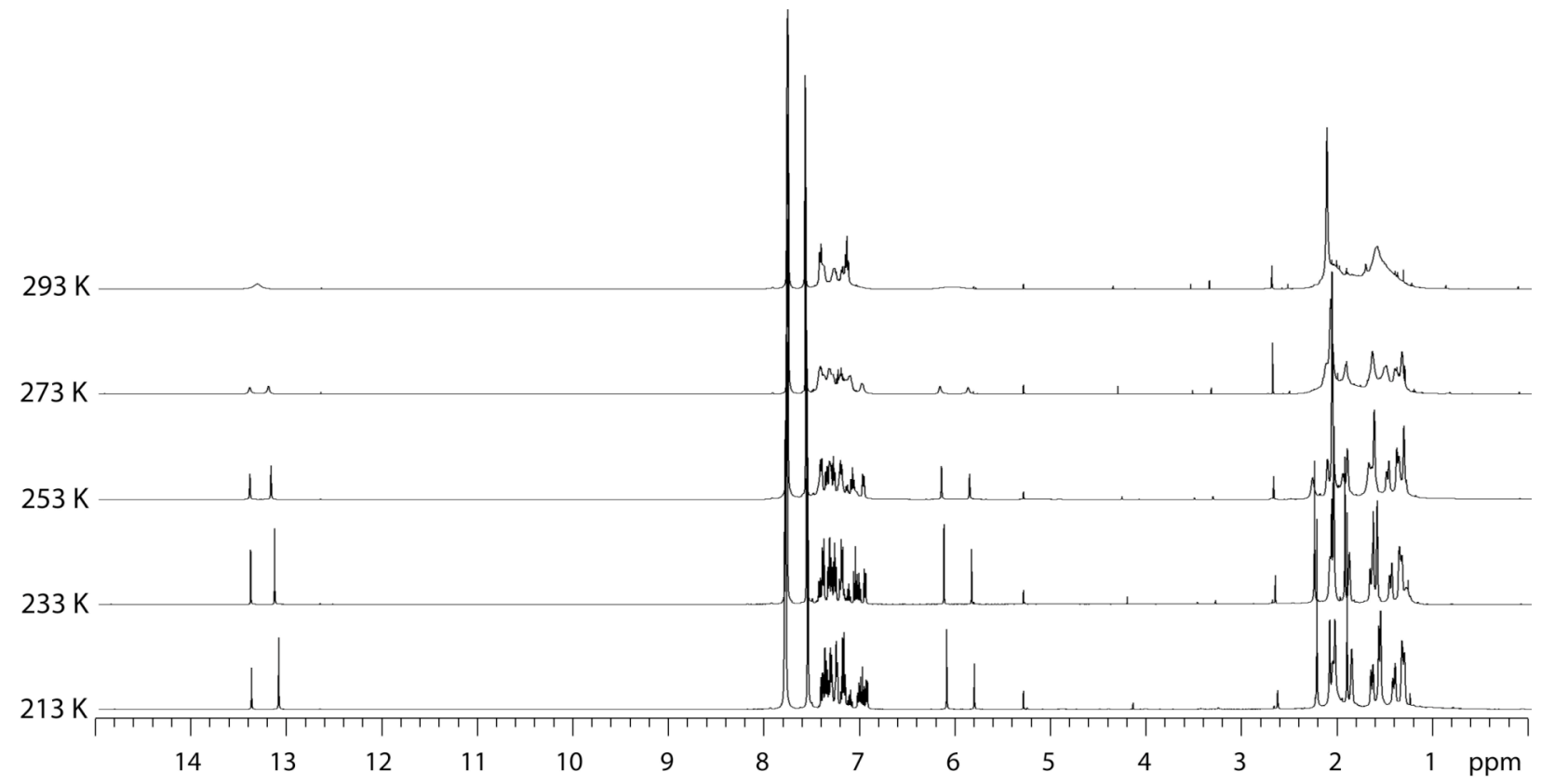

$500 \mathrm{MHz}{ }^{1} \mathrm{H}$ NMR variable temperature spectra of $\mathbf{2 d}$ in methylene chloride- $d_{2}$. 


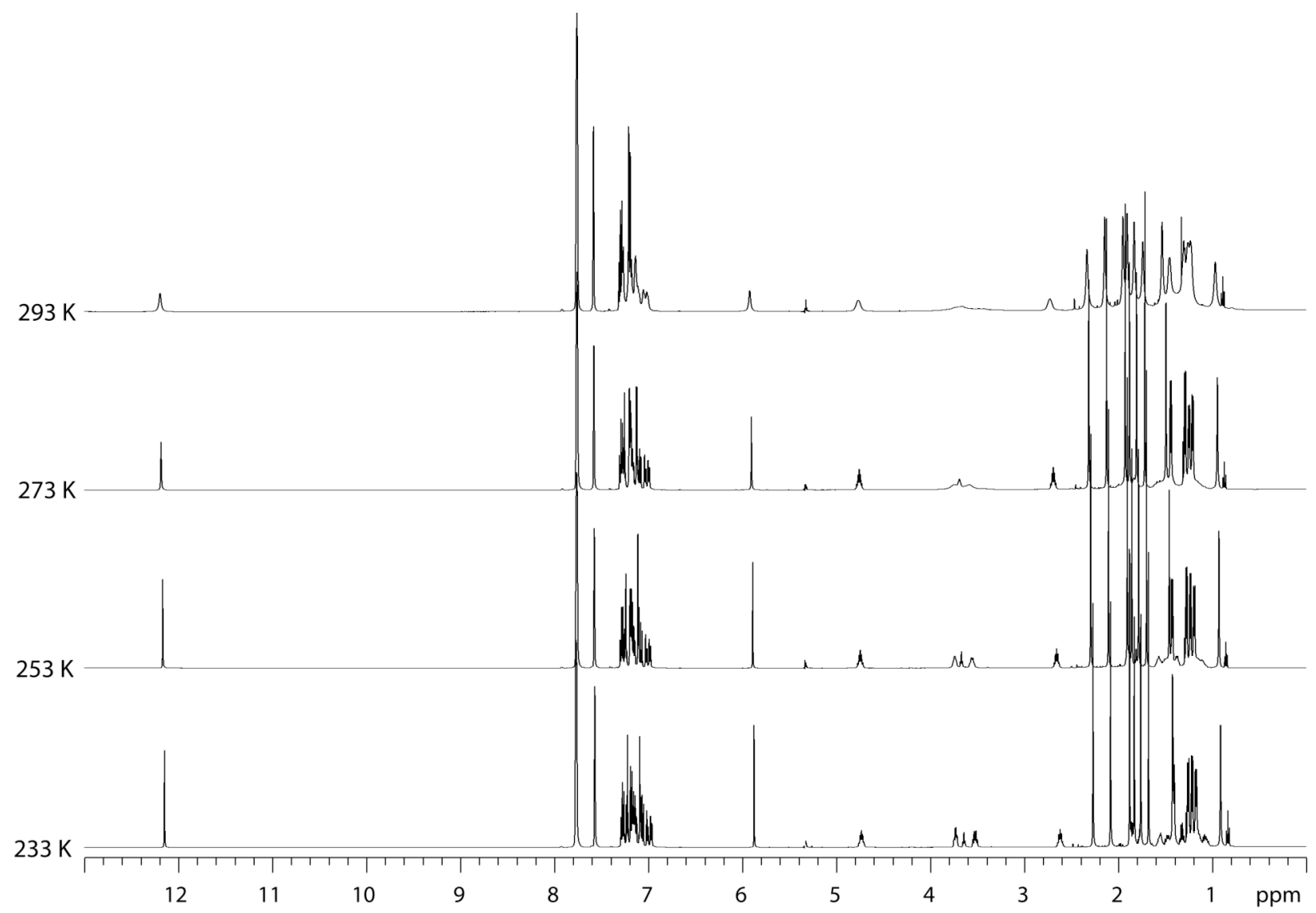

$500 \mathrm{MHz}{ }^{1} \mathrm{H}$ NMR variable temperature spectra of $\mathbf{4 a}$ in methylene chloride- $d_{2}$. 


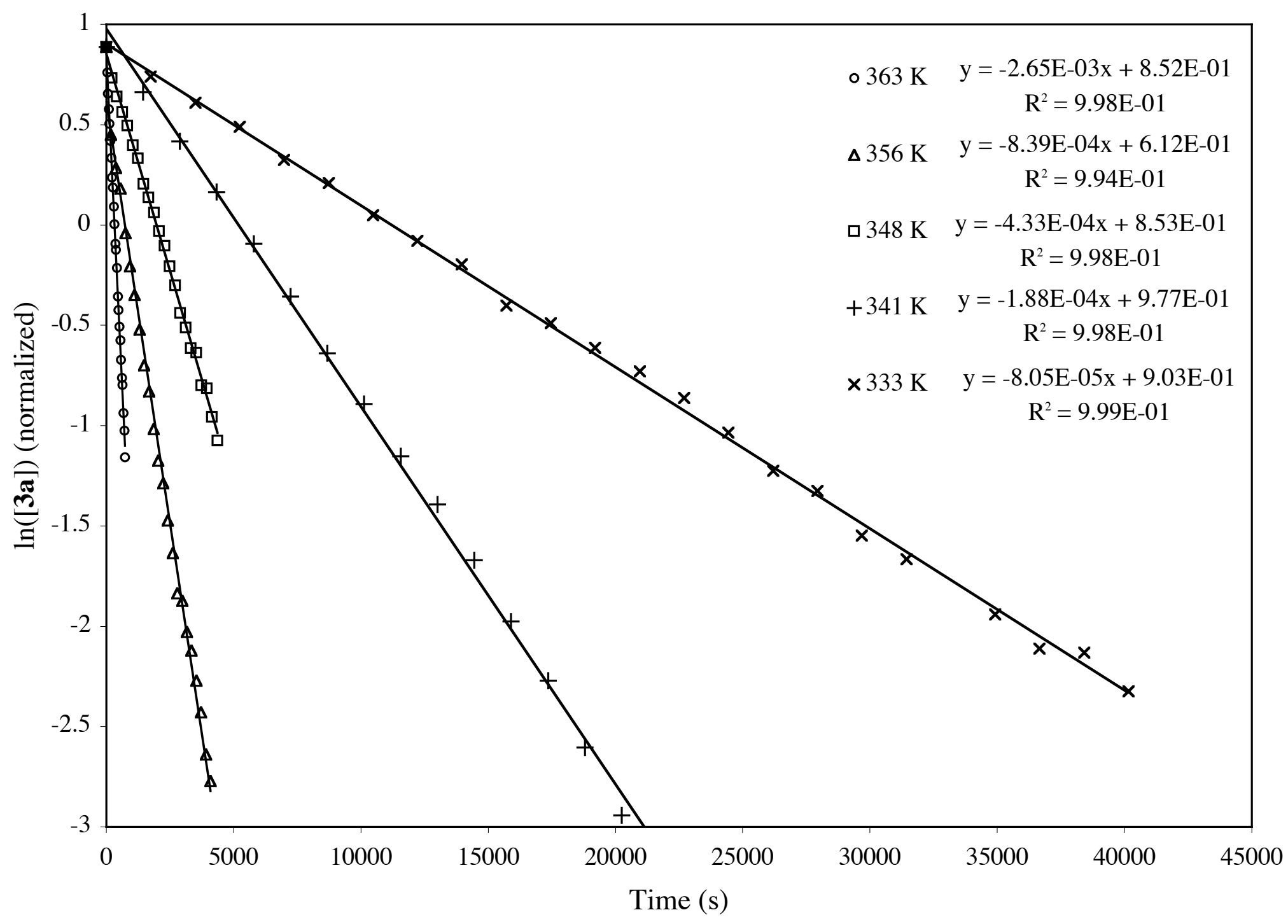

Kinetic plots of for the thermal reaction of 3a between $333 \mathrm{~K}$ and $363 \mathrm{~K}$ in toluene- $d_{8}$. 


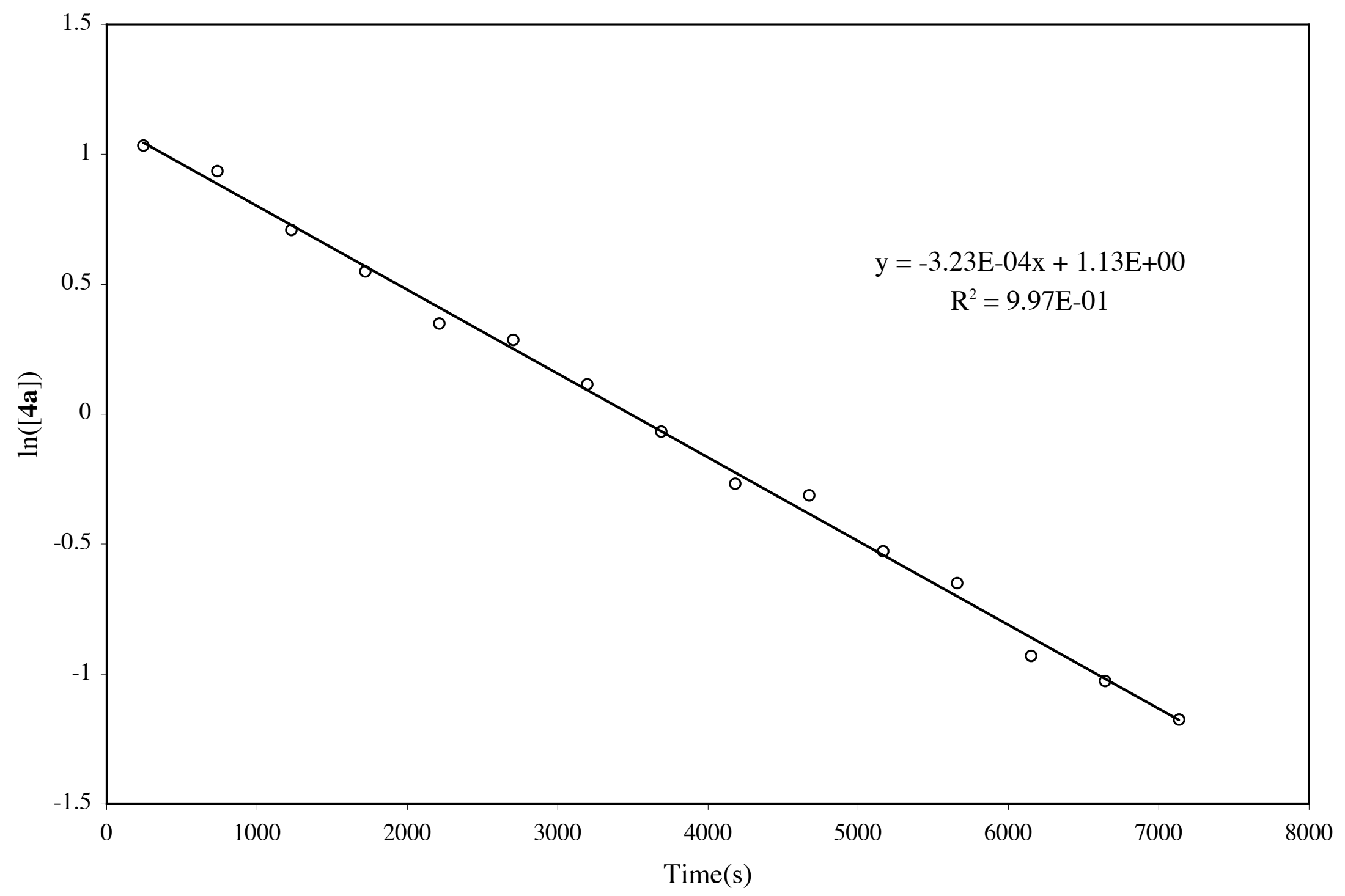

Kinetic plot for the thermal reaction of $\mathbf{4 a}$ at $313 \mathrm{~K}$ in methylene chloride- $d_{2}$. 


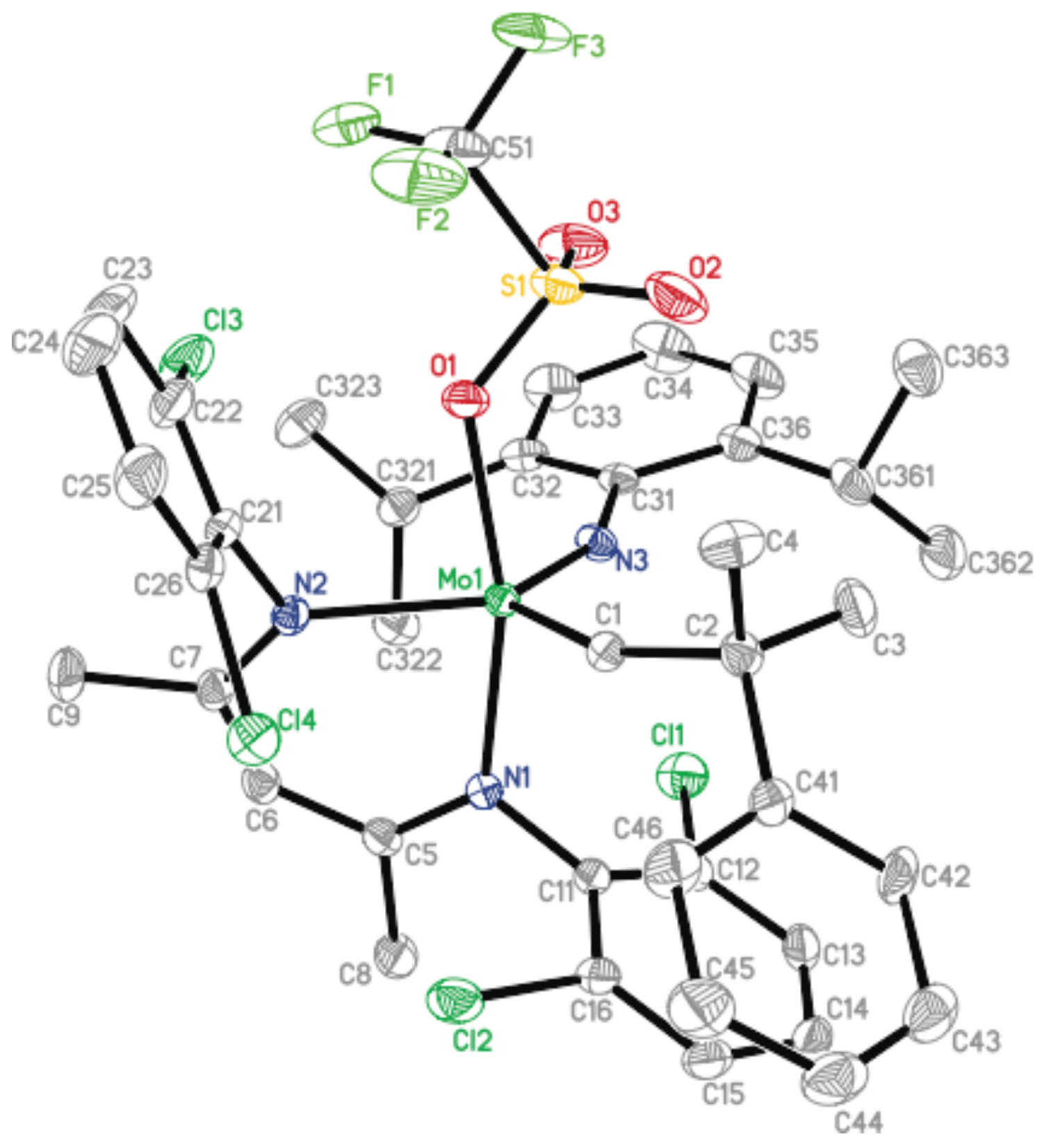

Fully labeled thermal ellipsoid drawing (35\%) of the syn component of 1a. Hydrogen atoms, cocyrstallized diethyl ether molecule, and disordered atoms omitted for clarity. 


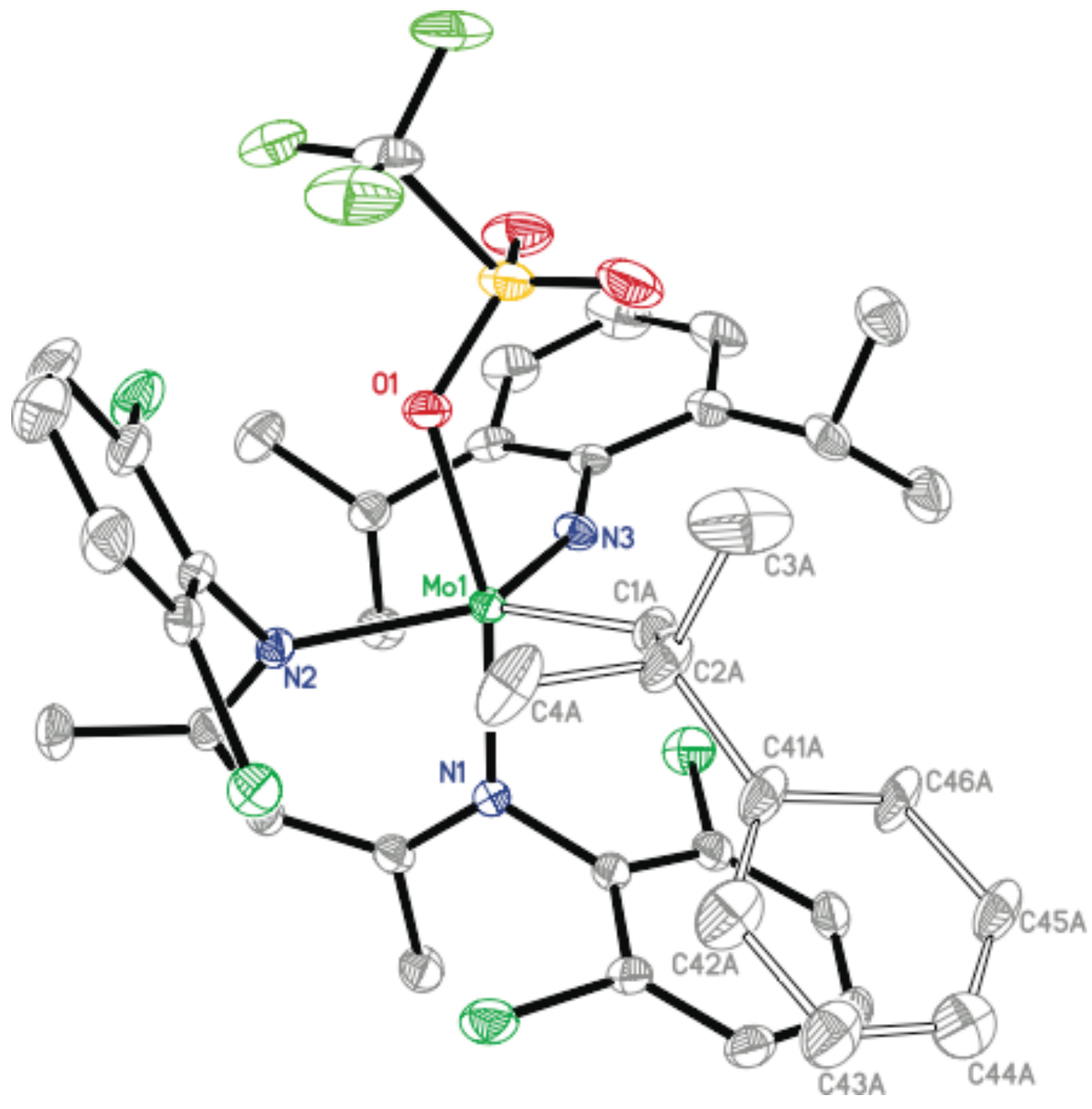

Partially labeled thermal ellipsoid drawing (35\%) of the anti component of 1a. Hydrogen atoms, cocrystallized diethyl ether molecule, and disordered atoms omitted for clarity. Atomic labels other than those of the neophylidene ligand are identical to syn component above. 
Crystal data and structure refinement for $\mathbf{1 a}$.

Identification code

Empirical formula

Formula weight

Temperature

Wavelength

Crystal system

Space group

Unit cell dimensions

Volume

$\mathrm{Z}$

Density (calculated)

Absorption coefficient

$\mathrm{F}(000)$

Crystal size

$\Theta$ range for data collection

Index ranges

Reflections collected

Independent reflections

Completeness to $\Theta=29.57^{\circ}$

Absorption correction

Max. and min. transmission

Refinement method

Data / restraints / parameters

Goodness-of-fit on $\mathrm{F}^{2}$

Final $\mathrm{R}$ indices $[\mathrm{I}>2 \sigma(\mathrm{I})]$

$\mathrm{R}$ indices (all data)

Largest diff. peak and hole
06066

$\mathrm{C}_{42} \mathrm{H}_{47} \mathrm{Cl}_{4} \mathrm{~F}_{3} \mathrm{MoN}_{3} \mathrm{O}_{3.50} \mathrm{~S}$

$976.63 \mathrm{~g} / \mathrm{mol}$

100(2) K

$0.71073 \AA$

Monoclinic

$\mathrm{P} 2 / \mathrm{n}$

$\mathrm{a}=11.0969(5) \AA \quad \alpha=90^{\circ}$

$\mathrm{b}=17.9753(8) \AA$

$\beta=92.2900(10)^{\circ}$

$\mathrm{c}=21.9851(8) \AA$

$\gamma=90^{\circ}$
4381.9(3) $\AA^{3}$

4

$1.480 \mathrm{~g} / \mathrm{cm}^{3}$

$0.647 \mathrm{~mm}^{-1}$

2004

$0.25 \times 0.20 \times 0.15 \mathrm{~mm}^{3}$

1.85 to $29.57^{\circ}$

$-15 \leq h \leq 15,-24 \leq k \leq 24,-30 \leq l \leq 30$

94631

$12298[\mathrm{R}(\mathrm{int})=0.0629]$

$100.0 \%$

Semi-empirical from equivalents

0.9092 and 0.8551

Full-matrix least-squares on $\mathrm{F}^{2}$

12298 / 1468 / 874

1.086

$\mathrm{R} 1=0.0374, \mathrm{wR} 2=0.0908$

$\mathrm{R} 1=0.0452, \mathrm{wR} 2=0.0947$

0.847 and $-0.489 \mathrm{e} \cdot \AA^{-3}$ 
Notes on the refinement of $\mathbf{1 a}$.

Much of the structure of compound 1a is disordered. The individual disorders do not seem to be coupled and the ratios were refined independently and freely. All disorders were refined with the help of similarity restraints on 1-2 and 1-3 distances. To counteract correlation effects arising from the low data to parameter ratio (ca. 8.4:1), similarity restraints on displacement parameters as well as rigid bond restraints for anisotropic displacement parameters were applied to all atoms. The structure is a mixture of two isomers, syn and anti. This mixture has been refined as a disorder and the fractional occupancy refined to 0.702 (4), corresponding to $70 \%$ 'syn' and $30 \%$ 'anti'. In spite of the disorder, the hydrogen atom bound to C(1) (major component, corresponding to the 'syn' isomer) could be clearly located in the difference Fourier synthesis and was refined semi-freely with the help of a distance restraint, while constraining its $U$-value to 1.2 times the $U_{e q}$ value of $\mathrm{C}(1)$. The hydrogen on the 'anti' isomer (atom $\mathrm{C}(1 \mathrm{~A})$ ) could not be located in the difference density and has been placed on its geometrically calculated position.

There is half a molecule of diethyl ether per asymmetric unit, located near a crystallographic inversion center. This results in a non-integer value for oxygen in the empirical formula. 
Atomic coordinates $\left(\times 10^{4}\right)$ and equivalent isotropic displacement parameters $\left(\AA^{2} \times 10^{3}\right)$ for 1a (06066). U(eq) is defined as one third of the trace of the orthogonalized $\mathrm{Uij}^{\mathrm{ij}}$ tensor.

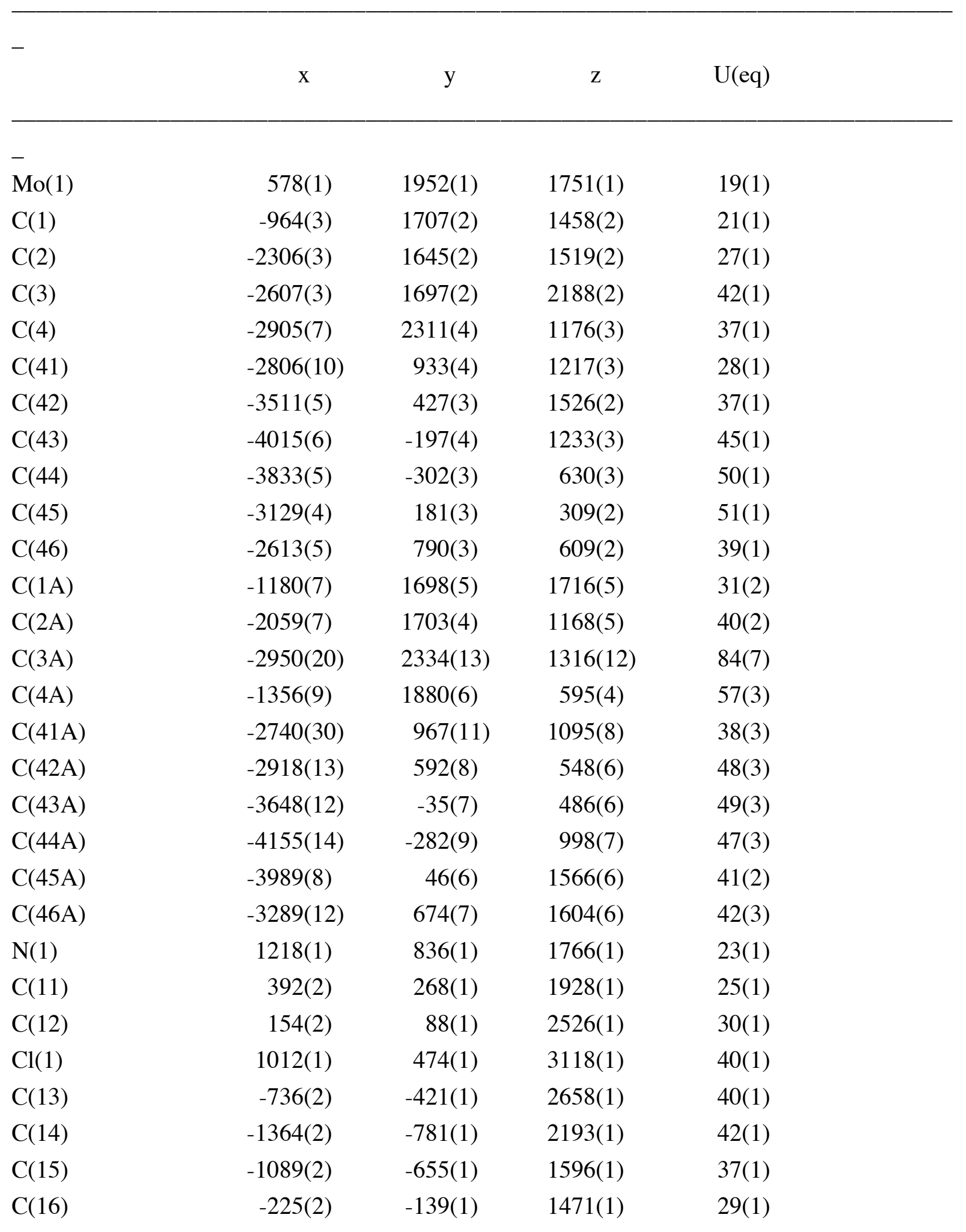




\begin{tabular}{|c|c|c|c|c|}
\hline $\mathrm{Cl}(2)$ & $165(1)$ & $5(1)$ & $723(1)$ & $39(1)$ \\
\hline$C(5)$ & $2313(2)$ & $609(1)$ & $1629(1)$ & $26(1)$ \\
\hline$C(6)$ & $3127(2)$ & $1050(1)$ & $1322(1)$ & $28(1)$ \\
\hline$C(7)$ & $2878(2)$ & $1700(1)$ & $1006(1)$ & $25(1)$ \\
\hline $\mathrm{C}(8)$ & $2696(2)$ & $-174(1)$ & $1782(1)$ & $38(1)$ \\
\hline $\mathrm{C}(9)$ & $3803(2)$ & 1941(1) & $560(1)$ & $37(1)$ \\
\hline $\mathrm{N}(2)$ & $1862(1)$ & $2096(1)$ & 1051(1) & $23(1)$ \\
\hline $\mathrm{C}(21)$ & $1673(5)$ & $2696(3)$ & $631(2)$ & $27(1)$ \\
\hline$C(22)$ & $2175(9)$ & $3401(4)$ & $718(3)$ & $37(1)$ \\
\hline $\mathrm{Cl}(3)$ & $3127(2)$ & $3542(1)$ & $1350(1)$ & $49(1)$ \\
\hline $\mathrm{C}(23)$ & 1939(10) & $3986(4)$ & $325(3)$ & $51(2)$ \\
\hline$C(24)$ & 1194(9) & $3870(4)$ & $-180(3)$ & $53(2)$ \\
\hline$C(25)$ & $699(5)$ & $3184(3)$ & $-294(3)$ & $43(1)$ \\
\hline$C(26)$ & $953(4)$ & $2604(2)$ & $106(2)$ & $27(1)$ \\
\hline $\mathrm{Cl}(4)$ & $400(1)$ & $1730(1)$ & $-82(1)$ & $32(1)$ \\
\hline $\mathrm{C}(21 \mathrm{~A})$ & 1769(9) & $2725(7)$ & $653(6)$ & $29(3)$ \\
\hline $\mathrm{C}(22 \mathrm{~A})$ & $1283(11)$ & $2687(7)$ & $59(6)$ & $31(3)$ \\
\hline $\mathrm{Cl}(3 \mathrm{~A})$ & $983(3)$ & $1815(2)$ & $-255(1)$ & $44(1)$ \\
\hline $\mathrm{C}(23 \mathrm{~A})$ & $1103(13)$ & $3315(8)$ & $-301(7)$ & $44(3)$ \\
\hline $\mathrm{C}(24 \mathrm{~A})$ & $1500(20)$ & $3978(10)$ & $-55(10)$ & $48(4)$ \\
\hline $\mathrm{C}(25 \mathrm{~A})$ & $2100(20)$ & $4021(11)$ & $498(8)$ & $46(4)$ \\
\hline$C(26 A)$ & 2209(19) & $3406(9)$ & $867(7)$ & $30(3)$ \\
\hline $\mathrm{Cl}(4 \mathrm{~A})$ & $2976(5)$ & $3487(3)$ & $1569(2)$ & $43(1)$ \\
\hline $\mathrm{N}(3)$ & $642(2)$ & $2073(1)$ & $2535(1)$ & $24(1)$ \\
\hline $\mathrm{C}(31)$ & $1139(5)$ & $2331(3)$ & $3099(2)$ & $23(1)$ \\
\hline $\mathrm{C}(32)$ & $2376(4)$ & $2511(2)$ & $3180(2)$ & $28(1)$ \\
\hline $\mathrm{C}(321)$ & $3277(6)$ & $2337(4)$ & $2707(3)$ & $29(1)$ \\
\hline $\mathrm{C}(322)$ & $3834(12)$ & $1569(5)$ & $2848(7)$ & $35(2)$ \\
\hline $\mathrm{C}(323)$ & $4272(7)$ & 2921(4) & $2660(3)$ & $45(1)$ \\
\hline $\mathrm{C}(33)$ & $2760(4)$ & $2825(3)$ & $3731(2)$ & $43(1)$ \\
\hline $\mathrm{C}(34)$ & $1990(5)$ & $2942(3)$ & $4195(2)$ & $52(1)$ \\
\hline $\mathrm{C}(35)$ & $804(5)$ & $2712(3)$ & $4131(2)$ & $41(1)$ \\
\hline$C(36)$ & $359(5)$ & 2399(3) & $3591(3)$ & $29(1)$ \\
\hline $\mathrm{C}(361)$ & $-920(6)$ & $2117(4)$ & $3545(2)$ & $34(1)$ \\
\hline$C(362)$ & $-1139(5)$ & $1544(3)$ & $4049(2)$ & $54(1)$ \\
\hline$C(363)$ & $-1803(10)$ & $2752(6)$ & $3582(6)$ & $45(2)$ \\
\hline
\end{tabular}




$\begin{array}{lclll}\mathrm{C}(31 \mathrm{~A}) & 984(8) & 2153(4) & 3145(4) & 28(2) \\ \mathrm{C}(32 \mathrm{~A}) & 2223(7) & 2204(5) & 3290(3) & 41(2) \\ \mathrm{C}(324) & 3195(10) & 2147(6) & 2831(5) & 39(2) \\ \mathrm{C}(325) & 4045(11) & 2811(7) & 2868(7) & 58(3) \\ \mathrm{C}(326) & 3920(20) & 1427(10) & 2897(14) & 53(5) \\ \mathrm{C}(33 \mathrm{~A}) & 2532(7) & 2284(7) & 3903(4) & 72(3) \\ \mathrm{C}(34 \mathrm{~A}) & 1674(8) & 2307(8) & 4338(4) & 87(4) \\ \mathrm{C}(35 \mathrm{~A}) & 463(8) & 2268(7) & 4172(3) & 66(3) \\ \mathrm{C}(36 \mathrm{~A}) & 78(8) & 2199(6) & 3575(4) & 33(2) \\ \mathrm{C}(364) & -1234(8) & 2159(7) & 3385(5) & 40(2) \\ \mathrm{C}(365) & -2011(17) & 2726(9) & 3703(10) & 48(4) \\ \mathrm{C}(366) & -1709(7) & 1378(4) & 3513(5) & 68(3) \\ \mathrm{O}(1) & 321(2) & 3127(1) & 1591(1) & 31(1) \\ \mathrm{S}(1) & -273(1) & 3657(1) & 2000(1) & 34(1) \\ \mathrm{O}(2) & -1512(8) & 3506(8) & 2017(6) & 54(2) \\ \mathrm{O}(3) & 357(12) & 3807(7) & 2562(4) & 49(2) \\ \mathrm{C}(51) & -126(10) & 4504(4) & 1557(3) & 47(2) \\ \mathrm{F}(1) & 994(9) & 4726(3) & 1519(3) & 62(2) \\ \mathrm{F}(2) & -597(11) & 4413(3) & 1000(2) & 71(2) \\ \mathrm{F}(3) & -744(10) & 5045(3) & 1826(3) & 62(2) \\ \mathrm{O}(2 \mathrm{~A}) & -1439(10) & 3451(10) & 2195(6) & 43(2) \\ \mathrm{O}(3 \mathrm{~A}) & 562(15) & 3934(12) & 2452(7) & 58(3) \\ \mathrm{C}(51 \mathrm{~A}) & -641(13) & 4449(5) & 1496(5) & 54(3) \\ \mathrm{F}(1 \mathrm{~A}) & -1295(13) & 4943(5) & 1788(5) & 69(3) \\ \mathrm{F}(2 \mathrm{~A}) & -1266(15) & 4251(6) & 1004(3) & 81(3) \\ \mathrm{F}(3 \mathrm{~A}) & 359(15) & 4769(3) & 1327(7) & 79(4) \\ \mathrm{C}(2 \mathrm{~S}) & 6292(6) & 6156(4) & 206(4) & 70(2) \\ \mathrm{C}(1 \mathrm{~S}) & 5957(4) & -162(3) & 61(2) \\ \mathrm{O}(1 \mathrm{~S}) & 5370(2) & 126(2) & 47(1) \\ \mathrm{C}(4 \mathrm{~S}) & 5129(5) & -191(3) & 64(2) \\ & 498(7) & 193(5) & 91(3)\end{array}$


Bond lengths $[\AA ̊]$ and angles $\left[^{\circ}\right]$ for $\mathbf{1 a}(\mathbf{0 6 0 6 6})$.

\begin{tabular}{|c|c|}
\hline- & \\
\hline Mo(1)-N(3) & $1.7356(16)$ \\
\hline $\operatorname{Mo}(1)-C(1)$ & $1.858(3)$ \\
\hline $\operatorname{Mo}(1)-C(1 A)$ & $2.002(8)$ \\
\hline $\operatorname{Mo}(1)-N(1)$ & $2.1266(16)$ \\
\hline $\mathrm{Mo}(1)-\mathrm{N}(2)$ & $2.1547(16)$ \\
\hline $\mathrm{Mo}(1)-\mathrm{O}(1)$ & $2.1593(14)$ \\
\hline $\mathrm{C}(1)-\mathrm{C}(2)$ & $1.505(4)$ \\
\hline$C(2)-C(3)$ & $1.525(5)$ \\
\hline$C(2)-C(41)$ & $1.534(5)$ \\
\hline$C(2)-C(4)$ & $1.550(7)$ \\
\hline$C(41)-C(46)$ & $1.388(7)$ \\
\hline$C(41)-C(42)$ & $1.394(7)$ \\
\hline$C(42)-C(43)$ & $1.400(7)$ \\
\hline$C(43)-C(44)$ & $1.362(8)$ \\
\hline$C(44)-C(45)$ & $1.381(7)$ \\
\hline$C(45)-C(46)$ & $1.389(5)$ \\
\hline$C(1 \mathrm{~A})-\mathrm{C}(2 \mathrm{~A})$ & $1.520(11)$ \\
\hline$C(2 A)-C(41 A)$ & $1.528(11)$ \\
\hline $\mathrm{C}(2 \mathrm{~A})-\mathrm{C}(4 \mathrm{~A})$ & $1.540(12)$ \\
\hline$C(2 A)-C(3 A)$ & $1.550(15)$ \\
\hline $\mathrm{C}(41 \mathrm{~A})-\mathrm{C}(42 \mathrm{~A})$ & $1.385(15)$ \\
\hline$C(41 \mathrm{~A})-\mathrm{C}(46 \mathrm{~A})$ & $1.400(16)$ \\
\hline $\mathrm{C}(42 \mathrm{~A})-\mathrm{C}(43 \mathrm{~A})$ & $1.392(13)$ \\
\hline $\mathrm{C}(43 \mathrm{~A})-\mathrm{C}(44 \mathrm{~A})$ & $1.354(14)$ \\
\hline$C(44 A)-C(45 A)$ & $1.386(13)$ \\
\hline $\mathrm{C}(45 \mathrm{~A})-\mathrm{C}(46 \mathrm{~A})$ & $1.371(10)$ \\
\hline $\mathrm{N}(1)-\mathrm{C}(5)$ & $1.328(2)$ \\
\hline $\mathrm{N}(1)-\mathrm{C}(11)$ & $1.428(2)$ \\
\hline $\mathrm{C}(11)-\mathrm{C}(12)$ & $1.390(3)$ \\
\hline$C(11)-C(16)$ & $1.398(3)$ \\
\hline$C(12)-C(13)$ & $1.386(3)$ \\
\hline $\mathrm{C}(12)-\mathrm{Cl}(1)$ & $1.726(2)$ \\
\hline $\mathrm{C}(13)-\mathrm{C}(14)$ & $1.375(4)$ \\
\hline
\end{tabular}




\begin{tabular}{|c|c|}
\hline$C(14)-C(15)$ & $1.379(4)$ \\
\hline$C(15)-C(16)$ & $1.370(3)$ \\
\hline $\mathrm{C}(16)-\mathrm{Cl}(2)$ & $1.735(2)$ \\
\hline$C(5)-C(6)$ & $1.396(3)$ \\
\hline$C(5)-C(8)$ & $1.502(3)$ \\
\hline$C(6)-C(7)$ & $1.382(3)$ \\
\hline$C(7)-N(2)$ & $1.340(3)$ \\
\hline$C(7)-C(9)$ & $1.511(3)$ \\
\hline $\mathrm{N}(2)-\mathrm{C}(21 \mathrm{~A})$ & $1.431(12)$ \\
\hline $\mathrm{N}(2)-\mathrm{C}(21)$ & $1.431(5)$ \\
\hline$C(21)-C(26)$ & $1.387(6)$ \\
\hline $\mathrm{C}(21)-\mathrm{C}(22)$ & $1.395(6)$ \\
\hline$C(22)-C(23)$ & $1.380(7)$ \\
\hline $\mathrm{C}(22)-\mathrm{Cl}(3)$ & $1.730(6)$ \\
\hline$C(23)-C(24)$ & $1.374(7)$ \\
\hline$C(24)-C(25)$ & $1.370(7)$ \\
\hline$C(25)-C(26)$ & $1.385(6)$ \\
\hline $\mathrm{C}(26)-\mathrm{Cl}(4)$ & $1.731(4)$ \\
\hline$C(21 A)-C(26 A)$ & $1.394(14)$ \\
\hline$C(21 A)-C(22 A)$ & $1.396(14)$ \\
\hline $\mathrm{C}(22 \mathrm{~A})-\mathrm{C}(23 \mathrm{~A})$ & $1.388(13)$ \\
\hline $\mathrm{C}(22 \mathrm{~A})-\mathrm{Cl}(3 \mathrm{~A})$ & $1.741(12)$ \\
\hline $\mathrm{C}(23 \mathrm{~A})-\mathrm{C}(24 \mathrm{~A})$ & $1.373(14)$ \\
\hline $\mathrm{C}(24 \mathrm{~A})-\mathrm{C}(25 \mathrm{~A})$ & $1.365(13)$ \\
\hline$C(25 A)-C(26 A)$ & $1.373(14)$ \\
\hline $\mathrm{C}(26 \mathrm{~A})-\mathrm{Cl}(4 \mathrm{~A})$ & $1.738(12)$ \\
\hline $\mathrm{N}(3)-\mathrm{C}(31 \mathrm{~A})$ & $1.386(9)$ \\
\hline $\mathrm{N}(3)-\mathrm{C}(31)$ & $1.415(6)$ \\
\hline $\mathrm{C}(31)-\mathrm{C}(32)$ & $1.415(6)$ \\
\hline$C(31)-C(36)$ & $1.416(6)$ \\
\hline $\mathrm{C}(32)-\mathrm{C}(33)$ & $1.388(5)$ \\
\hline $\mathrm{C}(32)-\mathrm{C}(321)$ & $1.504(6)$ \\
\hline $\mathrm{C}(321)-\mathrm{C}(323)$ & $1.530(8)$ \\
\hline $\mathrm{C}(321)-\mathrm{C}(322)$ & $1.538(9)$ \\
\hline $\mathrm{C}(33)-\mathrm{C}(34)$ & $1.374(6)$ \\
\hline$C(34)-C(35)$ & $1.381(7)$ \\
\hline
\end{tabular}




$\begin{array}{ll}\mathrm{C}(35)-\mathrm{C}(36) & 1.387(6) \\ \mathrm{C}(36)-\mathrm{C}(361) & 1.507(7) \\ \mathrm{C}(361)-\mathrm{C}(363) & 1.508(10) \\ \mathrm{C}(361)-\mathrm{C}(362) & 1.540(7) \\ \mathrm{C}(31 \mathrm{~A})-\mathrm{C}(32 \mathrm{~A}) & 1.402(10) \\ \mathrm{C}(31 \mathrm{~A})-\mathrm{C}(36 \mathrm{~A}) & 1.409(10) \\ \mathrm{C}(32 \mathrm{~A})-\mathrm{C}(33 \mathrm{~A}) & 1.386(9) \\ \mathrm{C}(32 \mathrm{~A})-\mathrm{C}(324) & 1.508(10) \\ \mathrm{C}(324)-\mathrm{C}(325) & 1.520(12) \\ \mathrm{C}(324)-\mathrm{C}(326) & 1.530(14) \\ \mathrm{C}(33 \mathrm{~A})-\mathrm{C}(34 \mathrm{~A}) & 1.376(11) \\ \mathrm{C}(34 \mathrm{~A})-\mathrm{C}(35 \mathrm{~A}) & 1.381(11) \\ \mathrm{C}(35 \mathrm{~A})-\mathrm{C}(36 \mathrm{~A}) & 1.370(10) \\ \mathrm{C}(36 \mathrm{~A})-\mathrm{C}(364) & 1.501(10) \\ \mathrm{C}(364)-\mathrm{C}(365) & 1.523(14) \\ \mathrm{C}(364)-\mathrm{C}(366) & 1.530(13) \\ \mathrm{O}(1)-\mathrm{S}(1) & 1.4825(15) \\ \mathrm{S}(1)-\mathrm{O}(2) & 1.404(8) \\ \mathrm{S}(1)-\mathrm{O}(3) & 1.420(7) \\ \mathrm{S}(1)-\mathrm{O}(3 \mathrm{~A}) & 1.422(10) \\ \mathrm{S}(1)-\mathrm{O}(2 \mathrm{~A}) & 1.427(9) \\ \mathrm{S}(1)-\mathrm{C}(51) & 1.819(7) \\ \mathrm{S}(1)-\mathrm{C}(51 \mathrm{~A}) & 1.840(8) \\ \mathrm{C}(51)-\mathrm{F}(1) & 1.312(7) \\ \mathrm{C}(51)-\mathrm{F}(2) & 1.322(7) \\ \mathrm{C}(51)-\mathrm{F}(3) & 1.341(7) \\ \mathrm{C}(51 \mathrm{~A})-\mathrm{F}(2 \mathrm{~A}) & 1.311(10) \\ \mathrm{C}(51 \mathrm{~A})-\mathrm{F}(3 \mathrm{~A}) & 1.317(8) \\ \mathrm{C}(51 \mathrm{~A})-\mathrm{F}(1 \mathrm{~A}) & 1.328(9) \\ \mathrm{C}(2 \mathrm{~S})-\mathrm{C}(1 \mathrm{~S}) & 1.489(9) \\ \mathrm{C}(1 \mathrm{~S})-\mathrm{O}(1 \mathrm{~S}) & 1.405(7) \\ \mathrm{O}(1 \mathrm{~S})-\mathrm{C}(3 \mathrm{~S}) & 1.413(7) \\ \mathrm{C}(3 \mathrm{~S})-\mathrm{C}(4 \mathrm{~S}) & 1.514(11) \\ \mathrm{N}(3)-\mathrm{Mo}(1)-\mathrm{C}(1) & \\ \mathrm{N}(3)-\mathrm{Mo}(1)-\mathrm{C}(1 \mathrm{~A}) & \\ & \\ & \\ & \end{array}$




\begin{tabular}{|c|c|}
\hline$C(1)-M o(1)-C(1 A)$ & $18.2(2)$ \\
\hline $\mathrm{N}(3)-\mathrm{Mo}(1)-\mathrm{N}(1)$ & $95.94(7)$ \\
\hline $\mathrm{C}(1)-\mathrm{Mo}(1)-\mathrm{N}(1)$ & $94.81(11)$ \\
\hline $\mathrm{C}(1 \mathrm{~A})-\mathrm{Mo}(1)-\mathrm{N}(1)$ & $96.3(2)$ \\
\hline $\mathrm{N}(3)-\mathrm{Mo}(1)-\mathrm{N}(2)$ & $133.84(7)$ \\
\hline $\mathrm{C}(1)-\mathrm{Mo}(1)-\mathrm{N}(2)$ & $113.99(12)$ \\
\hline $\mathrm{C}(1 \mathrm{~A})-\mathrm{Mo}(1)-\mathrm{N}(2)$ & $132.1(3)$ \\
\hline $\mathrm{N}(1)-\mathrm{Mo}(1)-\mathrm{N}(2)$ & $83.91(6)$ \\
\hline $\mathrm{N}(3)-\mathrm{Mo}(1)-\mathrm{O}(1)$ & $92.26(7)$ \\
\hline $\mathrm{C}(1)-\mathrm{Mo}(1)-\mathrm{O}(1)$ & $93.50(11)$ \\
\hline $\mathrm{C}(1 \mathrm{~A})-\mathrm{Mo}(1)-\mathrm{O}(1)$ & $95.4(2)$ \\
\hline $\mathrm{N}(1)-\mathrm{Mo}(1)-\mathrm{O}(1)$ & $165.19(6)$ \\
\hline $\mathrm{N}(2)-\mathrm{Mo}(1)-\mathrm{O}(1)$ & $81.52(6)$ \\
\hline $\mathrm{C}(2)-\mathrm{C}(1)-\mathrm{Mo}(1)$ & $152.6(3)$ \\
\hline$C(1)-C(2)-C(3)$ & $109.7(3)$ \\
\hline$C(1)-C(2)-C(41)$ & $111.4(5)$ \\
\hline$C(3)-C(2)-C(41)$ & $112.3(4)$ \\
\hline$C(1)-C(2)-C(4)$ & $107.8(4)$ \\
\hline$C(3)-C(2)-C(4)$ & $108.4(3)$ \\
\hline$C(41)-C(2)-C(4)$ & $107.1(5)$ \\
\hline$C(46)-C(41)-C(42)$ & $117.4(4)$ \\
\hline$C(46)-C(41)-C(2)$ & $120.3(5)$ \\
\hline$C(42)-C(41)-C(2)$ & $122.3(5)$ \\
\hline$C(41)-C(42)-C(43)$ & $121.3(5)$ \\
\hline $\mathrm{C}(44)-\mathrm{C}(43)-\mathrm{C}(42)$ & $119.2(5)$ \\
\hline$C(43)-C(44)-C(45)$ & $121.2(5)$ \\
\hline$C(44)-C(45)-C(46)$ & $119.0(5)$ \\
\hline$C(41)-C(46)-C(45)$ & $121.8(5)$ \\
\hline $\mathrm{C}(2 \mathrm{~A})-\mathrm{C}(1 \mathrm{~A})-\mathrm{Mo}(1)$ & $128.5(7)$ \\
\hline$C(1 \mathrm{~A})-\mathrm{C}(2 \mathrm{~A})-\mathrm{C}(41 \mathrm{~A})$ & $112.1(11)$ \\
\hline$C(1 A)-C(2 A)-C(4 A)$ & $108.7(7)$ \\
\hline$C(41 A)-C(2 A)-C(4 A)$ & $110.9(9)$ \\
\hline$C(1 \mathrm{~A})-\mathrm{C}(2 \mathrm{~A})-\mathrm{C}(3 \mathrm{~A})$ & $103.5(12)$ \\
\hline$C(41 \mathrm{~A})-\mathrm{C}(2 \mathrm{~A})-\mathrm{C}(3 \mathrm{~A})$ & $109.8(15)$ \\
\hline$C(4 A)-C(2 A)-C(3 A)$ & $111.7(12)$ \\
\hline $\mathrm{C}(42 \mathrm{~A})-\mathrm{C}(41 \mathrm{~A})-\mathrm{C}(46 \mathrm{~A})$ & $117.2(10)$ \\
\hline
\end{tabular}




\begin{tabular}{|c|c|}
\hline $\mathrm{C}(42$ & 1 \\
\hline$C(46 A)-C(41 A)-C(2 A)$ & $118.1(11)$ \\
\hline$C(41 A)-C(42 A)-C(43 A)$ & $122.9(12)$ \\
\hline$C(44 A)-C(43 A)-C(42 A)$ & $116.3(13)$ \\
\hline $\mathrm{C}(43 \mathrm{~A})-\mathrm{C}(44 \mathrm{~A})-\mathrm{C}(45 \mathrm{~A})$ & $124.4(14)$ \\
\hline$C(46 A)-C(45 A)-C(44 A)$ & $117.4(12)$ \\
\hline$C(45 A)-C(46 A)-C(41 A)$ & $121.7(11)$ \\
\hline $\mathrm{C}(5)-\mathrm{N}(1)-\mathrm{C}(11)$ & $116.00(16)$ \\
\hline $\mathrm{C}(5)-\mathrm{N}(1)-\mathrm{Mo}(1)$ & $126.51(13)$ \\
\hline $\mathrm{C}(11)-\mathrm{N}(1)-\mathrm{Mo}(1)$ & $8(12)$ \\
\hline$C(12)-C(11)-C(16)$ & 116.91(19) \\
\hline $\mathrm{C}(12)-\mathrm{C}(11)-\mathrm{N}(1)$ & $123.41(19)$ \\
\hline$C(16)-C(11)-N(1)$ & $119.68(18)$ \\
\hline $\mathrm{C}(13)-\mathrm{C}(12)-\mathrm{C}(11)$ & $121.0(2)$ \\
\hline $\mathrm{C}(13)-\mathrm{C}(12)-\mathrm{Cl}(1)$ & $118.98(18)$ \\
\hline $\mathrm{C}(11)-\mathrm{C}(12)-\mathrm{Cl}(1)$ & $119.99(16)$ \\
\hline$C(14)-C(13)-C(12)$ & $119.9(2)$ \\
\hline$C(13)-C(14)-C(15)$ & $120.4(2)$ \\
\hline$C(16)-C(15)-C(14)$ & $119.0(2)$ \\
\hline$C(15)-C(16)-C(11)$ & $122.4(2)$ \\
\hline $\mathrm{C}(15)-\mathrm{C}(16)-\mathrm{Cl}(2)$ & $119.52(18)$ \\
\hline $\mathrm{C}(11)-\mathrm{C}(16)-\mathrm{Cl}(2)$ & $118.01(16)$ \\
\hline $\mathrm{N}(1)-\mathrm{C}(5)-\mathrm{C}(6)$ & $123.35(19)$ \\
\hline $\mathrm{N}(1)-\mathrm{C}(5)-\mathrm{C}(8)$ & $119.42(18)$ \\
\hline$C(6)-C(5)-C(8)$ & $117.16(18)$ \\
\hline$C(7)-C(6)-C(5)$ & $127.12(19)$ \\
\hline $\mathrm{N}(2)-\mathrm{C}(7)-\mathrm{C}(6)$ & $124.29(18)$ \\
\hline $\mathrm{N}(2)-\mathrm{C}(7)-\mathrm{C}(9)$ & $119.36(19)$ \\
\hline$C(6)-C(7)-C(9)$ & $116.26(19)$ \\
\hline $\mathrm{C}(7)-\mathrm{N}(2)-\mathrm{C}(21 \mathrm{~A})$ & $114.5(4)$ \\
\hline $\mathrm{C}(7)-\mathrm{N}(2)-\mathrm{C}(21)$ & $117.1(2)$ \\
\hline $\mathrm{C}(21 \mathrm{~A})-\mathrm{N}(2)-\mathrm{C}(21)$ & $5.1(8)$ \\
\hline $\mathrm{C}(7)-\mathrm{N}(2)-\mathrm{Mo}(1)$ & $124.84(13)$ \\
\hline $\mathrm{C}(21 \mathrm{~A})-\mathrm{N}(2)-\mathrm{Mo}(1)$ & $119.9(4)$ \\
\hline $\mathrm{C}(21)-\mathrm{N}(2)-\mathrm{Mo}(1)$ & $117.88(19)$ \\
\hline$C(26)-C(21)-C(22)$ & $115.9(4)$ \\
\hline
\end{tabular}




\begin{tabular}{|c|c|}
\hline $\mathrm{C}(26)-\mathrm{C}(21)-\mathrm{N}(2)$ & $120.8(4)$ \\
\hline $\mathrm{C}(22)-\mathrm{C}(21)-\mathrm{N}(2)$ & $123.3(5)$ \\
\hline $\mathrm{C}(23)-\mathrm{C}(22)-\mathrm{C}(21)$ & $122.8(5)$ \\
\hline $\mathrm{C}(23)-\mathrm{C}(22)-\mathrm{Cl}(3)$ & $119.1(5)$ \\
\hline $\mathrm{C}(21)-\mathrm{C}(22)-\mathrm{Cl}(3)$ & $118.1(5)$ \\
\hline $\mathrm{C}(24)-\mathrm{C}(23)-\mathrm{C}(22)$ & $118.9(5)$ \\
\hline $\mathrm{C}(25)-\mathrm{C}(24)-\mathrm{C}(23)$ & $120.6(5)$ \\
\hline$C(24)-C(25)-C(26)$ & $119.5(5)$ \\
\hline $\mathrm{C}(25)-\mathrm{C}(26)-\mathrm{C}(21)$ & $122.3(4)$ \\
\hline $\mathrm{C}(25)-\mathrm{C}(26)-\mathrm{Cl}(4)$ & $118.1(4)$ \\
\hline $\mathrm{C}(21)-\mathrm{C}(26)-\mathrm{Cl}(4)$ & $119.6(3)$ \\
\hline$C(26 A)-C(21 A)-C(22 A)$ & $118.4(10)$ \\
\hline $\mathrm{C}(26 \mathrm{~A})-\mathrm{C}(21 \mathrm{~A})-\mathrm{N}(2)$ & $118.3(12)$ \\
\hline $\mathrm{C}(22 \mathrm{~A})-\mathrm{C}(21 \mathrm{~A})-\mathrm{N}(2)$ & $123.4(12)$ \\
\hline$C(23 A)-C(22 A)-C(21 A)$ & $122.4(11)$ \\
\hline $\mathrm{C}(23 \mathrm{~A})-\mathrm{C}(22 \mathrm{~A})-\mathrm{Cl}(3 \mathrm{~A})$ & $119.1(11)$ \\
\hline $\mathrm{C}(21 \mathrm{~A})-\mathrm{C}(22 \mathrm{~A})-\mathrm{Cl}(3 \mathrm{~A})$ & $118.4(10)$ \\
\hline$C(24 A)-C(23 A)-C(22 A)$ & $116.4(13)$ \\
\hline$C(25 A)-C(24 A)-C(23 A)$ & $122.5(14)$ \\
\hline$C(24 A)-C(25 A)-C(26 A)$ & $120.5(15)$ \\
\hline$C(25 A)-C(26 A)-C(21 A)$ & $119.2(12)$ \\
\hline$C(25 A)-C(26 A)-C l(4 A)$ & $119.0(11)$ \\
\hline $\mathrm{C}(21 \mathrm{~A})-\mathrm{C}(26 \mathrm{~A})-\mathrm{Cl}(4 \mathrm{~A})$ & $121.7(11)$ \\
\hline $\mathrm{C}(31 \mathrm{~A})-\mathrm{N}(3)-\mathrm{C}(31)$ & $15.5(4)$ \\
\hline $\mathrm{C}(31 \mathrm{~A})-\mathrm{N}(3)-\mathrm{Mo}(1)$ & $166.5(4)$ \\
\hline $\mathrm{C}(31)-\mathrm{N}(3)-\mathrm{Mo}(1)$ & $155.6(3)$ \\
\hline $\mathrm{N}(3)-\mathrm{C}(31)-\mathrm{C}(32)$ & $121.8(4)$ \\
\hline$N(3)-C(31)-C(36)$ & $118.0(5)$ \\
\hline $\mathrm{C}(32)-\mathrm{C}(31)-\mathrm{C}(36)$ & $120.3(5)$ \\
\hline$C(33)-C(32)-C(31)$ & $117.7(4)$ \\
\hline $\mathrm{C}(33)-\mathrm{C}(32)-\mathrm{C}(321)$ & $119.9(5)$ \\
\hline $\mathrm{C}(31)-\mathrm{C}(32)-\mathrm{C}(321)$ & $122.3(5)$ \\
\hline$C(32)-C(321)-C(323)$ & $113.9(5)$ \\
\hline$C(32)-C(321)-C(322)$ & $108.7(6)$ \\
\hline$C(323)-C(321)-C(322)$ & $110.1(6)$ \\
\hline$C(34)-C(33)-C(32)$ & $122.2(4)$ \\
\hline
\end{tabular}




\begin{tabular}{|c|c|}
\hline$C(33)-C(34)-C(35)$ & $119.8(4)$ \\
\hline$C(34)-C(35)-C(36)$ & $120.9(4)$ \\
\hline$C(35)-C(36)-C(31)$ & $118.8(5)$ \\
\hline$C(35)-C(36)-C(361)$ & $119.7(5)$ \\
\hline$C(31)-C(36)-C(361)$ & $121.5(5)$ \\
\hline $\mathrm{C}(36)-\mathrm{C}(361)-\mathrm{C}(363)$ & $110.8(7)$ \\
\hline $\mathrm{C}(36)-\mathrm{C}(361)-\mathrm{C}(362)$ & $110.6(5)$ \\
\hline$C(363)-C(361)-C(362)$ & $110.2(6)$ \\
\hline $\mathrm{N}(3)-\mathrm{C}(31 \mathrm{~A})-\mathrm{C}(32 \mathrm{~A})$ & $117.0(7)$ \\
\hline$N(3)-C(31 A)-C(36 A)$ & $118.7(7)$ \\
\hline$C(32 A)-C(31 A)-C(36 A)$ & $124.3(8)$ \\
\hline$C(33 A)-C(32 A)-C(31 A)$ & $115.4(7)$ \\
\hline$C(33 A)-C(32 A)-C(324)$ & $120.1(7)$ \\
\hline $\mathrm{C}(31 \mathrm{~A})-\mathrm{C}(32 \mathrm{~A})-\mathrm{C}(324)$ & $124.4(8)$ \\
\hline $\mathrm{C}(32 \mathrm{~A})-\mathrm{C}(324)-\mathrm{C}(325)$ & $111.7(9)$ \\
\hline $\mathrm{C}(32 \mathrm{~A})-\mathrm{C}(324)-\mathrm{C}(326)$ & $112.6(12)$ \\
\hline$C(325)-C(324)-C(326)$ & $109.5(12)$ \\
\hline $\mathrm{C}(34 \mathrm{~A})-\mathrm{C}(33 \mathrm{~A})-\mathrm{C}(32 \mathrm{~A})$ & $121.9(7)$ \\
\hline$C(33 A)-C(34 A)-C(35 A)$ & $120.6(7)$ \\
\hline$C(36 A)-C(35 A)-C(34 A)$ & $121.3(8)$ \\
\hline$C(35 A)-C(36 A)-C(31 A)$ & $116.5(8)$ \\
\hline$C(35 A)-C(36 A)-C(364)$ & $122.1(8)$ \\
\hline$C(31 A)-C(36 A)-C(364)$ & $121.4(8)$ \\
\hline $\mathrm{C}(36 \mathrm{~A})-\mathrm{C}(364)-\mathrm{C}(365)$ & $113.6(11)$ \\
\hline $\mathrm{C}(36 \mathrm{~A})-\mathrm{C}(364)-\mathrm{C}(366)$ & 109.3(9) \\
\hline$C(365)-C(364)-C(366)$ & $109.0(10)$ \\
\hline $\mathrm{S}(1)-\mathrm{O}(1)-\mathrm{Mo}(1)$ & $126.01(9)$ \\
\hline $\mathrm{O}(2)-\mathrm{S}(1)-\mathrm{O}(3)$ & $117.5(6)$ \\
\hline $\mathrm{O}(2)-\mathrm{S}(1)-\mathrm{O}(3 \mathrm{~A})$ & 131.3(9) \\
\hline $\mathrm{O}(3)-\mathrm{S}(1)-\mathrm{O}(3 \mathrm{~A})$ & $16.5(10)$ \\
\hline $\mathrm{O}(2)-\mathrm{S}(1)-\mathrm{O}(2 \mathrm{~A})$ & $16.5(7)$ \\
\hline $\mathrm{O}(3)-\mathrm{S}(1)-\mathrm{O}(2 \mathrm{~A})$ & $102.0(7)$ \\
\hline $\mathrm{O}(3 \mathrm{~A})-\mathrm{S}(1)-\mathrm{O}(2 \mathrm{~A})$ & $117.0(8)$ \\
\hline $\mathrm{O}(2)-\mathrm{S}(1)-\mathrm{O}(1)$ & $110.6(5)$ \\
\hline $\mathrm{O}(3)-\mathrm{S}(1)-\mathrm{O}(1)$ & $115.8(7)$ \\
\hline $\mathrm{O}(3 \mathrm{~A})-\mathrm{S}(1)-\mathrm{O}(1)$ & $110.9(9)$ \\
\hline
\end{tabular}




$\begin{array}{lr}\mathrm{O}(2 \mathrm{~A})-\mathrm{S}(1)-\mathrm{O}(1) & 116.5(7) \\ \mathrm{O}(2)-\mathrm{S}(1)-\mathrm{C}(51) & 106.6(5) \\ \mathrm{O}(3)-\mathrm{S}(1)-\mathrm{C}(51) & 104.8(5) \\ \mathrm{O}(3 \mathrm{~A})-\mathrm{S}(1)-\mathrm{C}(51) & 90.6(7) \\ \mathrm{O}(2 \mathrm{~A})-\mathrm{S}(1)-\mathrm{C}(51) & 118.6(7) \\ \mathrm{O}(1)-\mathrm{S}(1)-\mathrm{C}(51) & 99.3(3) \\ \mathrm{O}(2)-\mathrm{S}(1)-\mathrm{C}(51 \mathrm{~A}) & 88.4(5) \\ \mathrm{O}(3)-\mathrm{S}(1)-\mathrm{C}(51 \mathrm{~A}) & 117.8(7) \\ \mathrm{O}(3 \mathrm{~A})-\mathrm{S}(1)-\mathrm{C}(51 \mathrm{~A}) & 105.8(7) \\ \mathrm{O}(2 \mathrm{~A})-\mathrm{S}(1)-\mathrm{C}(51 \mathrm{~A}) & 101.5(7) \\ \mathrm{O}(1)-\mathrm{S}(1)-\mathrm{C}(51 \mathrm{~A}) & 103.0(3) \\ \mathrm{C}(51)-\mathrm{S}(1)-\mathrm{C}(51 \mathrm{~A}) & 18.5(3) \\ \mathrm{F}(1)-\mathrm{C}(51)-\mathrm{F}(2) & 108.6(6) \\ \mathrm{F}(1)-\mathrm{C}(51)-\mathrm{F}(3) & 108.1(5) \\ \mathrm{F}(2)-\mathrm{C}(51)-\mathrm{F}(3) & 107.9(5) \\ \mathrm{F}(1)-\mathrm{C}(51)-\mathrm{S}(1) & 113.2(4) \\ \mathrm{F}(2)-\mathrm{C}(51)-\mathrm{S}(1) & 110.6(5) \\ \mathrm{F}(3)-\mathrm{C}(51)-\mathrm{S}(1) & 108.3(5) \\ \mathrm{F}(2 \mathrm{~A})-\mathrm{C}(51 \mathrm{~A})-\mathrm{F}(3 \mathrm{~A}) & 108.1(8) \\ \mathrm{F}(2 \mathrm{~A})-\mathrm{C}(51 \mathrm{~A})-\mathrm{F}(1 \mathrm{~A}) & 107.4(8) \\ \mathrm{F}(3 \mathrm{~A})-\mathrm{C}(51 \mathrm{~A})-\mathrm{F}(1 \mathrm{~A}) & 109.1(7) \\ \mathrm{F}(2 \mathrm{~A})-\mathrm{C}(51 \mathrm{~A})-\mathrm{S}(1) & 112.6(7) \\ \mathrm{F}(3 \mathrm{~A})-\mathrm{C}(51 \mathrm{~A})-\mathrm{S}(1) & 109.8(6) \\ \mathrm{F}(1 \mathrm{~A})-\mathrm{C}(51 \mathrm{~A})-\mathrm{S}(1) & 109.8(7) \\ \mathrm{O}(1 \mathrm{~S})-\mathrm{C}(1 \mathrm{~S})-\mathrm{C}(2 \mathrm{~S}) & 108.9(5) \\ \mathrm{C}(1 \mathrm{~S})-\mathrm{O}(1 \mathrm{~S})-\mathrm{C}(3 \mathrm{~S}) & 113.6(5) \\ \mathrm{O}(1 \mathrm{~S})-\mathrm{C}(3 \mathrm{~S})-\mathrm{C}(4 \mathrm{~S}) & 107.6(5) \\ & \end{array}$


Anisotropic displacement parameters $\left(\AA^{2} \times 10^{3}\right)$ for 1a (06066). The anisotropic displacement factor exponent takes the form: $-2 \mathrm{p}^{2}\left[h^{2} \mathrm{a}^{* 2} \mathrm{U}^{11}+\ldots+2 h k \mathrm{a}^{*} \mathrm{~b}^{*} \mathrm{U}^{12}\right]$
$\mathrm{U}^{11}$
$\mathrm{U}^{22}$
$\mathrm{U}^{33}$
$\mathrm{U}^{23}$
$\mathrm{U}^{13}$
$\mathrm{U}^{12}$

\begin{tabular}{lcccccc}
\hline $\mathrm{Mo}(1)$ & $20(1)$ & $19(1)$ & $17(1)$ & $-2(1)$ & $1(1)$ & $-1(1)$ \\
$\mathrm{C}(1)$ & $22(1)$ & $22(1)$ & $19(1)$ & $0(1)$ & $0(1)$ & $-1(1)$ \\
$\mathrm{C}(2)$ & $20(1)$ & $30(2)$ & $31(2)$ & $-2(1)$ & $0(1)$ & $2(1)$ \\
$\mathrm{C}(3)$ & $33(2)$ & $54(2)$ & $41(2)$ & $-10(2)$ & $15(1)$ & $0(2)$ \\
$\mathrm{C}(4)$ & $33(3)$ & $28(2)$ & $49(2)$ & $2(2)$ & $-16(2)$ & $3(2)$ \\
$\mathrm{C}(41)$ & $15(2)$ & $29(2)$ & $38(2)$ & $1(2)$ & $-2(2)$ & $2(2)$ \\
$\mathrm{C}(42)$ & $15(2)$ & $37(3)$ & $61(2)$ & $8(2)$ & $7(2)$ & $-3(2)$ \\
$\mathrm{C}(43)$ & $26(3)$ & $36(3)$ & $72(4)$ & $7(3)$ & $2(3)$ & $0(2)$ \\
$\mathrm{C}(44)$ & $32(3)$ & $36(3)$ & $79(4)$ & $-11(3)$ & $-5(2)$ & $-4(2)$ \\
$\mathrm{C}(45)$ & $40(2)$ & $57(3)$ & $57(2)$ & $-23(2)$ & $-5(2)$ & $-10(2)$ \\
$\mathrm{C}(46)$ & $31(3)$ & $47(3)$ & $39(2)$ & $-7(2)$ & $-7(2)$ & $-10(2)$ \\
$\mathrm{C}(1 \mathrm{~A})$ & $24(3)$ & $24(3)$ & $44(5)$ & $8(4)$ & $4(3)$ & $1(2)$ \\
$\mathrm{C}(2 \mathrm{~A})$ & $17(3)$ & $34(4)$ & $67(6)$ & $10(4)$ & $-8(3)$ & $-7(3)$ \\
$\mathrm{C}(3 \mathrm{~A})$ & $45(9)$ & $61(9)$ & $143(19)$ & $-24(11)$ & $-47(9)$ & $24(7)$ \\
$\mathrm{C}(4 \mathrm{~A})$ & $56(6)$ & $59(6)$ & $53(5)$ & $25(4)$ & $-17(4)$ & $-26(5)$ \\
$\mathrm{C}(41 \mathrm{~A})$ & $17(5)$ & $33(5)$ & $66(8)$ & $10(6)$ & $-2(7)$ & $-6(4)$ \\
$\mathrm{C}(42 \mathrm{~A})$ & $35(7)$ & $51(8)$ & $57(6)$ & $6(5)$ & $-17(5)$ & $-6(5)$ \\
$\mathrm{C}(43 \mathrm{~A})$ & $26(6)$ & $40(7)$ & $80(7)$ & $7(5)$ & $-1(5)$ & $2(5)$ \\
$\mathrm{C}(44 \mathrm{~A})$ & $19(5)$ & $46(6)$ & $76(9)$ & $1(6)$ & $-11(6)$ & $10(4)$ \\
$\mathrm{C}(45 \mathrm{~A})$ & $17(4)$ & $32(5)$ & $73(6)$ & $14(4)$ & $-3(4)$ & $-4(3)$ \\
$\mathrm{C}(46 \mathrm{~A})$ & $10(4)$ & $39(7)$ & $77(6)$ & $12(5)$ & $-6(4)$ & $-9(4)$ \\
$\mathrm{N}(1)$ & $23(1)$ & $21(1)$ & $24(1)$ & $-1(1)$ & $4(1)$ & $-1(1)$ \\
$\mathrm{C}(11)$ & $24(1)$ & $20(1)$ & $31(1)$ & $0(1)$ & $6(1)$ & $3(1)$ \\
$\mathrm{C}(12)$ & $32(1)$ & $24(1)$ & $33(1)$ & $1(1)$ & $9(1)$ & $5(1)$ \\
$\mathrm{Cl}(1)$ & $56(1)$ & $36(1)$ & $28(1)$ & $5(1)$ & $0(1)$ & $1(1)$ \\
$\mathrm{C}(13)$ & $40(1)$ & $32(1)$ & $49(1)$ & $6(1)$ & $24(1)$ & $3(1)$ \\
$\mathrm{C}(14)$ & $28(1)$ & $28(1)$ & $71(2)$ & $1(1)$ & $18(1)$ & $-2(1)$ \\
$\mathrm{C}(15)$ & $27(1)$ & $25(1)$ & $58(2)$ & $-7(1)$ & $1(1)$ & $0(1)$ \\
$\mathrm{C}(16)$ & $29(1)$ & $21(1)$ & $35(1)$ & $-4(1)$ & $4(1)$ & $1(1)$ \\
& & & & & &
\end{tabular}




\begin{tabular}{|c|c|c|c|c|c|c|}
\hline $\mathrm{Cl}(2)$ & $53(1)$ & $34(1)$ & $31(1)$ & $-8(1)$ & $0(1)$ & $-5(1)$ \\
\hline$C(5)$ & $25(1)$ & $26(1)$ & $27(1)$ & $-3(1)$ & $2(1)$ & $2(1)$ \\
\hline$C(6)$ & $20(1)$ & $33(1)$ & $30(1)$ & $-4(1)$ & $2(1)$ & $-1(1)$ \\
\hline$C(7)$ & $22(1)$ & $30(1)$ & $24(1)$ & $-5(1)$ & $3(1)$ & $-7(1)$ \\
\hline$C(8)$ & $30(1)$ & $30(1)$ & $55(1)$ & $5(1)$ & $12(1)$ & $9(1)$ \\
\hline $\mathrm{C}(9)$ & $26(1)$ & $45(1)$ & $40(1)$ & $2(1)$ & $11(1)$ & $-6(1)$ \\
\hline $\mathrm{N}(2)$ & $23(1)$ & $24(1)$ & $21(1)$ & $-2(1)$ & $1(1)$ & $-6(1)$ \\
\hline $\mathrm{C}(21)$ & 31(3) & $22(2)$ & $28(2)$ & $0(2)$ & $10(2)$ & $-6(2)$ \\
\hline$C(22)$ & $49(3)$ & $34(2)$ & $29(2)$ & $2(2)$ & $7(2)$ & $-16(2)$ \\
\hline $\mathrm{Cl}(3)$ & $63(1)$ & $43(1)$ & $42(1)$ & $-7(1)$ & $2(1)$ & $-30(1)$ \\
\hline$C(23)$ & $75(5)$ & $30(2)$ & $47(4)$ & $12(2)$ & $14(3)$ & $-15(2)$ \\
\hline$C(24)$ & $78(6)$ & $42(3)$ & $40(3)$ & $21(2)$ & $11(3)$ & $0(3)$ \\
\hline$C(25)$ & $53(3)$ & $47(2)$ & $30(2)$ & $13(2)$ & $6(2)$ & $1(2)$ \\
\hline$C(26)$ & $30(3)$ & $30(2)$ & $23(1)$ & $4(1)$ & $7(2)$ & $-2(2)$ \\
\hline $\mathrm{Cl}(4)$ & $41(1)$ & $37(1)$ & $19(1)$ & $-2(1)$ & $-5(1)$ & $-5(1)$ \\
\hline$C(21 A)$ & $14(6)$ & $43(6)$ & $29(6)$ & $4(5)$ & $-2(5)$ & $-2(5)$ \\
\hline$C(22 A)$ & $20(6)$ & $38(5)$ & $36(5)$ & $5(4)$ & $13(4)$ & $-8(4)$ \\
\hline $\mathrm{Cl}(3 \mathrm{~A})$ & $36(2)$ & $61(2)$ & $34(1)$ & $-6(1)$ & $4(1)$ & $-9(1)$ \\
\hline$C(23 A)$ & $41(8)$ & $51(6)$ & $41(5)$ & $22(5)$ & $0(6)$ & $5(6)$ \\
\hline $\mathrm{C}(24 \mathrm{~A})$ & $53(10)$ & $40(6)$ & $50(9)$ & $17(6)$ & $6(6)$ & $0(6)$ \\
\hline $\mathrm{C}(25 \mathrm{~A})$ & $39(6)$ & $50(7)$ & $49(9)$ & $17(6)$ & $4(7)$ & $-13(5)$ \\
\hline$C(26 A)$ & $29(6)$ & $26(5)$ & $35(7)$ & $9(4)$ & $9(5)$ & $-6(4)$ \\
\hline $\mathrm{Cl}(4 \mathrm{~A})$ & $50(2)$ & $32(1)$ & $47(2)$ & $-4(2)$ & $-10(2)$ & $-19(1)$ \\
\hline $\mathrm{N}(3)$ & $29(1)$ & $23(1)$ & 19(1) & $-1(1)$ & $0(1)$ & $5(1)$ \\
\hline$C(31)$ & $38(2)$ & $15(2)$ & $15(2)$ & $2(1)$ & $1(1)$ & $4(2)$ \\
\hline $\mathrm{C}(32)$ & $38(2)$ & $20(2)$ & $23(2)$ & $0(1)$ & $-9(1)$ & $1(2)$ \\
\hline $\mathrm{C}(321)$ & $31(2)$ & $28(3)$ & $29(2)$ & $-1(2)$ & $-2(2)$ & $-3(2)$ \\
\hline $\mathrm{C}(322)$ & $30(3)$ & $37(3)$ & $36(3)$ & $-7(3)$ & $-5(2)$ & $0(3)$ \\
\hline $\mathrm{C}(323)$ & $41(3)$ & $40(3)$ & $53(4)$ & $1(2)$ & $-4(2)$ & $-17(2)$ \\
\hline $\mathrm{C}(33)$ & $52(2)$ & $45(2)$ & $30(2)$ & $-7(2)$ & $-15(2)$ & $-1(2)$ \\
\hline$C(34)$ & $71(3)$ & $60(3)$ & $24(2)$ & $-16(2)$ & $-15(2)$ & $9(2)$ \\
\hline$C(35)$ & $65(3)$ & $44(2)$ & $16(2)$ & $-5(2)$ & $1(2)$ & $14(2)$ \\
\hline$C(36)$ & $40(3)$ & $25(3)$ & $24(2)$ & $0(2)$ & $1(2)$ & $5(2)$ \\
\hline $\mathrm{C}(361)$ & $42(3)$ & $40(2)$ & $22(2)$ & $-2(2)$ & $8(2)$ & $10(2)$ \\
\hline $\mathrm{C}(362)$ & $60(3)$ & $48(3)$ & $56(3)$ & $13(2)$ & $26(2)$ & $9(2)$ \\
\hline$C(363)$ & $40(5)$ & $53(4)$ & $42(4)$ & $7(2)$ & $5(3)$ & $12(3)$ \\
\hline
\end{tabular}




\begin{tabular}{|c|c|c|c|c|c|c|}
\hline$C(31 \mathrm{~A})$ & $33(3)$ & $25(4)$ & $24(3)$ & $-2(3)$ & $-6(2)$ & $2(3)$ \\
\hline$C(32 A)$ & $36(3)$ & $47(5)$ & $39(4)$ & $-20(4)$ & $-7(3)$ & $2(3)$ \\
\hline$C(324)$ & $31(3)$ & $40(6)$ & $45(6)$ & $-10(4)$ & $-7(3)$ & $-14(4)$ \\
\hline$C(325)$ & $41(5)$ & $46(5)$ & $87(10)$ & $-11(6)$ & $-4(5)$ & $-10(4)$ \\
\hline$C(326)$ & $41(6)$ & $47(7)$ & $70(11)$ & $-15(7)$ & $-3(6)$ & $8(5)$ \\
\hline$C(33 A)$ & $40(4)$ & 129(9) & $48(4)$ & $-38(5)$ & $-18(3)$ & $15(5)$ \\
\hline $\mathrm{C}(34 \mathrm{~A})$ & $59(5)$ & $163(11)$ & $39(4)$ & $-50(6)$ & $-11(3)$ & $18(6)$ \\
\hline$C(35 A)$ & $54(4)$ & $109(8)$ & $33(3)$ & $-28(5)$ & $2(3)$ & $11(5)$ \\
\hline$C(36 \mathrm{~A})$ & $38(4)$ & $40(6)$ & $22(3)$ & $-9(3)$ & $3(3)$ & $2(3)$ \\
\hline$C(364)$ & $27(4)$ & $49(4)$ & $44(6)$ & $-5(4)$ & $-2(3)$ & $0(3)$ \\
\hline$C(365)$ & $36(5)$ & $36(4)$ & $75(11)$ & $2(5)$ & $14(5)$ & $6(4)$ \\
\hline$C(366)$ & $42(4)$ & $38(4)$ & $125(9)$ & $-19(4)$ & $15(5)$ & $7(3)$ \\
\hline $\mathrm{O}(1)$ & $46(1)$ & $20(1)$ & $28(1)$ & $0(1)$ & $8(1)$ & $3(1)$ \\
\hline$S(1)$ & 47(1) & $24(1)$ & $32(1)$ & $-6(1)$ & $2(1)$ & $6(1)$ \\
\hline $\mathrm{O}(2)$ & 51(3) & $46(4)$ & $66(6)$ & $-28(4)$ & $10(3)$ & $5(2)$ \\
\hline $\mathrm{O}(3)$ & $80(5)$ & $33(3)$ & $35(3)$ & $-13(2)$ & $-8(3)$ & $3(3)$ \\
\hline$C(51)$ & $64(5)$ & $28(3)$ & $49(3)$ & $-2(2)$ & $-4(3)$ & $15(3)$ \\
\hline $\mathrm{F}(1)$ & $74(4)$ & $24(2)$ & $88(3)$ & $-3(2)$ & $15(3)$ & $-6(2)$ \\
\hline $\mathrm{F}(2)$ & $116(6)$ & $44(3)$ & $50(2)$ & $6(2)$ & $-25(3)$ & $29(3)$ \\
\hline $\mathrm{F}(3)$ & $74(4)$ & $26(2)$ & $86(3)$ & $-9(2)$ & $-1(3)$ & $19(2)$ \\
\hline $\mathrm{O}(2 \mathrm{~A})$ & $55(4)$ & $36(3)$ & $39(5)$ & $-4(4)$ & $22(3)$ & $12(3)$ \\
\hline $\mathrm{O}(3 \mathrm{~A})$ & $61(5)$ & $62(9)$ & $50(6)$ & $-24(5)$ & $-8(5)$ & $9(4)$ \\
\hline $\mathrm{C}(51 \mathrm{~A})$ & $67(7)$ & $30(4)$ & $65(5)$ & $18(3)$ & $17(4)$ & $16(4)$ \\
\hline $\mathrm{F}(1 \mathrm{~A})$ & $76(6)$ & $37(3)$ & $98(5)$ & $12(3)$ & $32(5)$ & $25(4)$ \\
\hline $\mathrm{F}(2 \mathrm{~A})$ & $106(8)$ & $72(5)$ & $64(3)$ & $21(3)$ & $-14(4)$ & $31(5)$ \\
\hline $\mathrm{F}(3 \mathrm{~A})$ & $88(7)$ & $31(2)$ & $120(7)$ & $16(3)$ & $50(6)$ & $10(3)$ \\
\hline $\mathrm{C}(2 \mathrm{~S})$ & $69(4)$ & $38(3)$ & $102(6)$ & $4(3)$ & $-6(4)$ & $0(3)$ \\
\hline$C(1 S)$ & $60(4)$ & $57(4)$ & $66(4)$ & $22(3)$ & $-14(3)$ & $-21(3)$ \\
\hline $\mathrm{O}(1 \mathrm{~S})$ & $48(2)$ & $39(2)$ & $53(2)$ & $1(2)$ & $10(2)$ & $-9(2)$ \\
\hline$C(3 S)$ & $53(3)$ & $72(5)$ & $69(5)$ & $-24(4)$ & $25(4)$ & $-22(3)$ \\
\hline$C(4 S)$ & $68(5)$ & $64(5)$ & 143(9) & $-10(5)$ & $43(6)$ & $-2(4)$ \\
\hline
\end{tabular}


Hydrogen coordinates $\left(\times 10^{4}\right)$ and isotropic displacement parameters $\left(\AA^{2} \times 10^{3}\right)$ for 1a (06066).






\begin{tabular}{|c|c|c|c|c|}
\hline $\mathrm{H}(8 \mathrm{~A})$ & 2532 & -279 & 2208 & 57 \\
\hline $\mathrm{H}(8 \mathrm{~B})$ & 3561 & -229 & 1720 & 57 \\
\hline $\mathrm{H}(8 \mathrm{C})$ & 2244 & -523 & 1517 & 57 \\
\hline $\mathrm{H}(9 \mathrm{~A})$ & 3454 & 1906 & 144 & 55 \\
\hline $\mathrm{H}(9 \mathrm{~B})$ & 4512 & 1618 & 601 & 55 \\
\hline $\mathrm{H}(9 \mathrm{C})$ & 4042 & 2457 & 645 & 55 \\
\hline $\mathrm{H}(23)$ & 2286 & 4462 & 403 & 61 \\
\hline $\mathrm{H}(24)$ & 1021 & 4269 & -453 & 64 \\
\hline $\mathrm{H}(25)$ & 185 & 3106 & -644 & 52 \\
\hline $\mathrm{H}(23 \mathrm{~A})$ & 726 & 3288 & -696 & 53 \\
\hline $\mathrm{H}(24 \mathrm{~A})$ & 1348 & 4423 & -278 & 57 \\
\hline $\mathrm{H}(25 \mathrm{~A})$ & 2447 & 4481 & 629 & 55 \\
\hline $\mathrm{H}(321)$ & 2834 & 2306 & 2303 & 35 \\
\hline $\mathrm{H}(32 \mathrm{~A})$ & 4419 & 1447 & 2541 & 52 \\
\hline $\mathrm{H}(32 \mathrm{~B})$ & 3196 & 1192 & 2841 & 52 \\
\hline $\mathrm{H}(32 \mathrm{C})$ & 4242 & 1579 & 3252 & 52 \\
\hline $\mathrm{H}(32 \mathrm{D})$ & 4819 & 2774 & 2342 & 68 \\
\hline $\mathrm{H}(32 \mathrm{E})$ & 4724 & 2959 & 3051 & 68 \\
\hline $\mathrm{H}(32 \mathrm{~F})$ & 3909 & 3404 & 2556 & 68 \\
\hline $\mathrm{H}(33)$ & 3583 & 2964 & 3789 & 51 \\
\hline $\mathrm{H}(34)$ & 2272 & 3180 & 4560 & 63 \\
\hline $\mathrm{H}(35)$ & 286 & 2769 & 4462 & 50 \\
\hline $\mathrm{H}(361)$ & -1053 & 1867 & 3141 & 41 \\
\hline $\mathrm{H}(36 \mathrm{~A})$ & -572 & 1130 & 4015 & 81 \\
\hline $\mathrm{H}(36 \mathrm{~B})$ & -1967 & 1357 & 4005 & 81 \\
\hline $\mathrm{H}(36 \mathrm{C})$ & -1017 & 1782 & 4448 & 81 \\
\hline$H(36 D)$ & -1664 & 3109 & 3255 & 68 \\
\hline $\mathrm{H}(36 \mathrm{E})$ & -1692 & 3000 & 3977 & 68 \\
\hline $\mathrm{H}(36 \mathrm{~F})$ & -2628 & 2558 & 3538 & 68 \\
\hline $\mathrm{H}(324)$ & 2791 & 2147 & 2417 & 47 \\
\hline $\mathrm{H}(32 \mathrm{G})$ & 4654 & 2759 & 2561 & 87 \\
\hline $\mathrm{H}(32 \mathrm{H})$ & 4442 & 2831 & 3274 & 87 \\
\hline $\mathrm{H}(32 \mathrm{I})$ & 3585 & 3269 & 2794 & 87 \\
\hline $\mathrm{H}(32 \mathrm{~J})$ & 4549 & 1420 & 2594 & 79 \\
\hline $\mathrm{H}(32 \mathrm{~K})$ & 3387 & 999 & 2833 & 79 \\
\hline $\mathrm{H}(32 \mathrm{~L})$ & 4306 & 1402 & 3307 & 79 \\
\hline
\end{tabular}




\begin{tabular}{lrrrr}
$\mathrm{H}(33 \mathrm{~A})$ & 3359 & 2325 & 4027 & 87 \\
$\mathrm{H}(34 \mathrm{~A})$ & 1918 & 2350 & 4756 & 105 \\
$\mathrm{H}(35 \mathrm{~A})$ & -116 & 2290 & 4479 & 79 \\
$\mathrm{H}(364)$ & -1315 & 2249 & 2937 & 48 \\
$\mathrm{H}(36 \mathrm{G})$ & -1751 & 3230 & 3598 & 73 \\
$\mathrm{H}(36 \mathrm{H})$ & -1925 & 2656 & 4144 & 73 \\
$\mathrm{H}(36 \mathrm{I})$ & -2858 & 2658 & 3570 & 73 \\
$\mathrm{H}(36 \mathrm{~J})$ & -1251 & 1011 & 3286 & 102 \\
$\mathrm{H}(36 \mathrm{~K})$ & -2564 & 1348 & 3386 & 102 \\
$\mathrm{H}(36 \mathrm{~L})$ & -1616 & 1273 & 3950 & 102 \\
$\mathrm{H}(2 \mathrm{~S} 1)$ & 1844 & 6565 & 8 & 105 \\
$\mathrm{H}(2 \mathrm{~S} 2)$ & 2578 & 6311 & 614 & 105 \\
$\mathrm{H}(2 \mathrm{~S} 3)$ & 1763 & 5722 & 238 & 105 \\
$\mathrm{H}(1 \mathrm{~S} 1)$ & 3061 & 5804 & -576 & 74 \\
$\mathrm{H}(1 \mathrm{~S} 2)$ & 3879 & 6394 & -199 & 74 \\
$\mathrm{H}(3 \mathrm{~S} 1)$ & 5538 & 5551 & -259 & 77 \\
$\mathrm{H}(3 \mathrm{~S} 2)$ & 4726 & 4920 & -592 & 77 \\
$\mathrm{H}(4 \mathrm{~S} 1)$ & 6333 & 4360 & -16 & 136 \\
$\mathrm{H}(4 \mathrm{~S} 2)$ & 5073 & 4123 & 256 & 136 \\
$\mathrm{H}(4 \mathrm{~S} 3)$ & 5881 & 4751 & 588 & 136 \\
& & & & \\
\hline
\end{tabular}




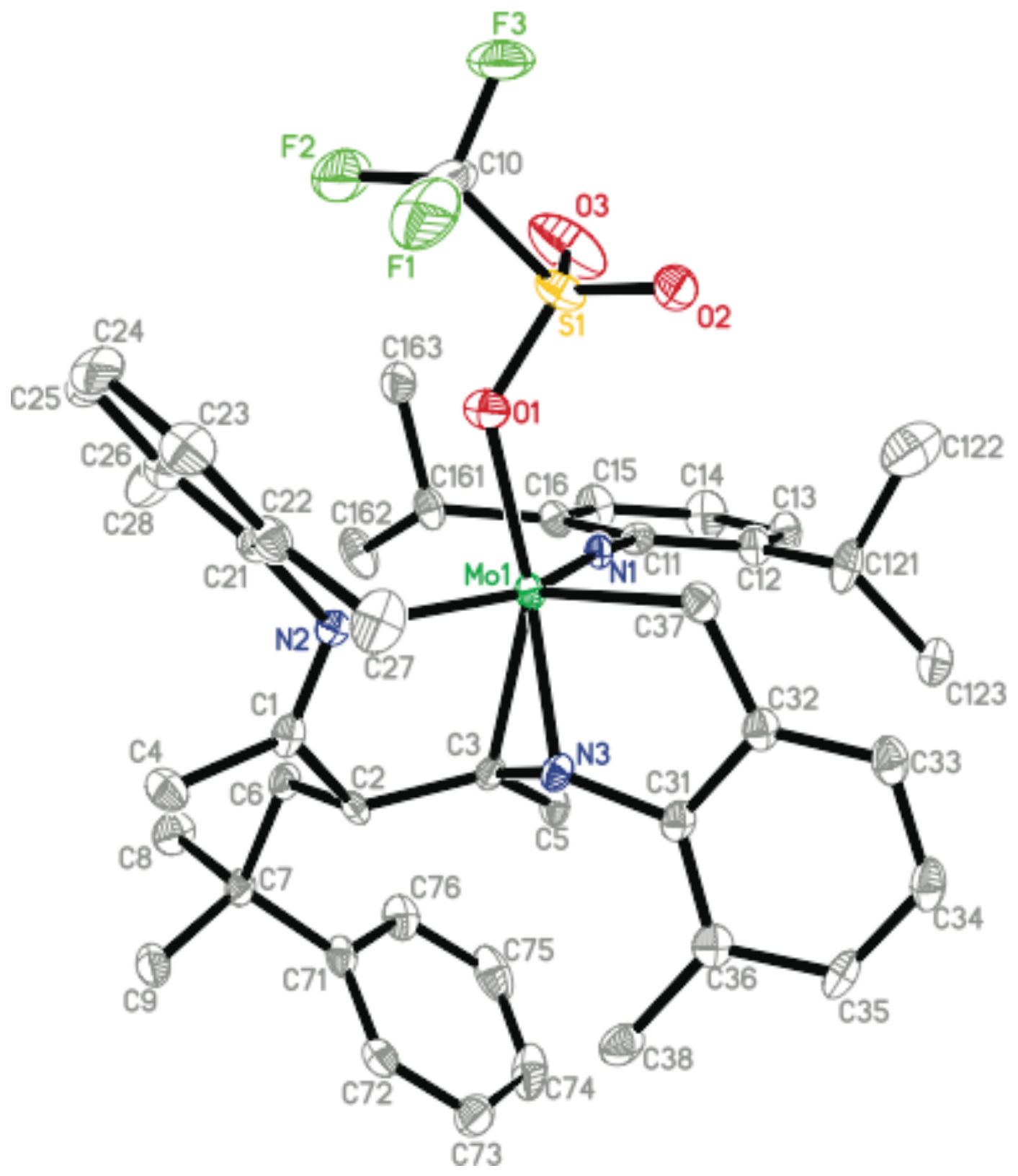

Fully labeled thermal ellipsoid drawing (35\%) of the first (Mo1) of two independent molecules of $\mathbf{3} \mathbf{a}^{\prime \prime}$ in the asymmetric unit. Hydrogen atoms omitted for clarity. 




Fully labeled thermal ellipsoid drawing (35\%) of the second (Mo2) of two independent molecules of $\mathbf{3} \mathbf{a}^{\prime \prime}$ in the asymmetric unit. Hydgrogen atoms omitted for clarity. 
Crystal data and structure refinement for $\mathbf{3} \mathbf{a}^{\prime \prime}$.

Identification code

Empirical formula

Formula weight

Temperature

Wavelength

Crystal system

Space group

Unit cell dimensions

Volume

$\mathrm{Z}$

Density (calculated)

Absorption coefficient

$\mathrm{F}(000)$

Crystal size

$\Theta$ range for data collection

Index ranges

Reflections collected

Independent reflections

Completeness to $\Theta=29.13^{\circ}$

Absorption correction

Max. and min. transmission

Refinement method

Data / restraints / parameters

Goodness-of-fit on $\mathrm{F}^{2}$

Final R indices [I $>2 \sigma(\mathrm{I})]$

$\mathrm{R}$ indices (all data)

Absolute structure parameter

Largest diff. peak and hole
06187

$\mathrm{C}_{44} \mathrm{H}_{54} \mathrm{~F}_{3} \mathrm{MoN}_{3} \mathrm{O}_{3} \mathrm{~S}$

$857.90 \mathrm{~g} / \mathrm{mol}$

100(2) K

$0.71073 \AA$

Monoclinic

$\mathrm{P} 2{ }_{1}$

$$
\begin{array}{ll}
\mathrm{a}=10.7550(5) \AA & \alpha=90^{\circ} \\
\mathrm{b}=17.4054(9) \AA & \beta=92.020(2)^{\circ} \\
\mathrm{c}=22.5561(12) \AA & \gamma=90^{\circ}
\end{array}
$$

4219.8(4) $\AA^{3}$

4

$1.350 \mathrm{~g} / \mathrm{cm}^{3}$

$0.415 \mathrm{~mm}^{-1}$

1792

$0.30 \times 0.10 \times 0.08 \mathrm{~mm}^{3}$

1.48 to $29.13^{\circ}$.

$-14 \leq h \leq 14,-23 \leq k \leq 23,0 \leq l \leq 30$

26565

26569

$99.5 \%$

Semi-empirical from equivalents

0.9675 and 0.8855

Full-matrix least-squares on $\mathrm{F}^{2}$

26569 / 986 / 1026

1.009

$\mathrm{R} 1=0.0389, \mathrm{wR} 2=0.0765$

$\mathrm{R} 1=0.0450, \mathrm{wR} 2=0.0782$

$-0.017(15)$

0.970 and $-0.647 \mathrm{e} \cdot \AA^{-3}$ 
Notes on the refinement of $\mathbf{3} \mathbf{a}^{\prime \prime}$.

Compound 3a" crystallizes as a non-merohedral twin with two molecules per asymmetric unit. Two independent orientation matrices for the unit cell were found using the program CELL_NOW ${ }^{1}$, and data reduction taking into account the twinning was performed with SAINT ${ }^{2}$. The program TWINABS ${ }^{3}$ was used to perform absorption correction and to set up the HKLF5 format file for structure refinement. The twin ratio was refined freely and converged at a value of 0.3240(5). Coordinates for the hydrogen atoms on the carbon atoms binding directly to the metal (C(37) and $\mathrm{C}(67))$ were taken from the difference Fourier synthesis and refined semifreely with the help of distance restraints, while constraining their $U$-values to 1.2 times the $U_{e q}$ value of the carbon atoms to which they bind. To counteract correlation effects arising from the twinning, similarity restraints on displacement parameters as well as rigid bond restraints for anisotropic displacement parameters were applied to all atoms.

${ }^{1}$ Sheldrick, G. M (2003). CELL_NOW, University of Göttingen, Germany.

${ }^{2}$ Bruker (2005). SAINT, Bruker-AXS Inc., Madison, Wisconsin, USA.

${ }^{3}$ Sheldrick, G. M (2002). TWINABS, University of Göttingen, Germany. 
Atomic coordinates $\left(\times 10^{4}\right)$ and equivalent isotropic displacement parameters $\left(\AA^{2} \times 10^{3}\right)$ for $3 \mathbf{a}^{\prime \prime}(\mathbf{0 6 1 8 7})$. U(eq) is defined as one third of the trace of the orthogonalized $\mathrm{U}^{\mathrm{ij}}$ tensor.






\begin{tabular}{|c|c|c|c|c|}
\hline$C(27)$ & $2594(3)$ & $3354(2)$ & $-1206(2)$ & $29(1)$ \\
\hline$C(28)$ & $3782(4)$ & $584(2)$ & $-786(2)$ & $30(1)$ \\
\hline $\mathrm{N}(3)$ & $1916(2)$ & 2995(1) & $499(1)$ & $14(1)$ \\
\hline $\mathrm{C}(31)$ & $1736(3)$ & $3677(2)$ & $824(2)$ & $15(1)$ \\
\hline$C(32)$ & $2777(3)$ & $4149(2)$ & $820(2)$ & $16(1)$ \\
\hline $\mathrm{C}(33)$ & $2775(3)$ & $4840(2)$ & $1135(2)$ & $20(1)$ \\
\hline$C(34)$ & $1727(3)$ & $5032(2)$ & $1443(2)$ & $22(1)$ \\
\hline $\mathrm{C}(35)$ & $683(3)$ & $4573(2)$ & $1421(2)$ & $20(1)$ \\
\hline$C(36)$ & $640(3)$ & $3883(2)$ & $1099(2)$ & $17(1)$ \\
\hline $\mathrm{C}(37)$ & $3796(3)$ & $3845(2)$ & $455(2)$ & $17(1)$ \\
\hline $\mathrm{C}(38)$ & $-526(3)$ & $3417(2)$ & $1042(2)$ & $23(1)$ \\
\hline $\mathrm{C}(1)$ & $1578(3)$ & $1769(2)$ & $-370(2)$ & $14(1)$ \\
\hline $\mathrm{C}(2)$ & $1115(3)$ & $1694(2)$ & $250(1)$ & $12(1)$ \\
\hline $\mathrm{C}(3)$ & $1820(2)$ & $2241(2)$ & $681(1)$ & $13(1)$ \\
\hline$C(4)$ & $727(3)$ & $1526(2)$ & $-874(2)$ & $21(1)$ \\
\hline$C(5)$ & $1746(3)$ & $2088(2)$ & $1338(2)$ & $16(1)$ \\
\hline$C(6)$ & $1260(3)$ & $813(2)$ & $409(1)$ & $16(1)$ \\
\hline$C(7)$ & $85(3)$ & $378(2)$ & $613(2)$ & $16(1)$ \\
\hline$C(8)$ & 497(3) & $-474(2)$ & $685(2)$ & $26(1)$ \\
\hline $\mathrm{C}(9)$ & $-956(3)$ & $393(2)$ & $136(2)$ & $21(1)$ \\
\hline$C(71)$ & $-339(3)$ & $702(2)$ & $1199(2)$ & $18(1)$ \\
\hline$C(72)$ & $-1286(3)$ & $1239(2)$ & $1223(2)$ & $19(1)$ \\
\hline$C(73)$ & $-1631(3)$ & $1566(2)$ & $1748(2)$ & $30(1)$ \\
\hline$C(74)$ & $-1026(4)$ & $1363(2)$ & $2276(2)$ & $33(1)$ \\
\hline$C(75)$ & $-65(4)$ & $841(2)$ & $2266(2)$ & $31(1)$ \\
\hline$C(76)$ & $264(3)$ & $501(2)$ & $1736(2)$ & $21(1)$ \\
\hline $\operatorname{Mo}(2)$ & $8440(1)$ & $7232(1)$ & $4655(1)$ & $11(1)$ \\
\hline $\mathrm{O}(4)$ & $10035(2)$ & $7274(1)$ & $5240(1)$ & $18(1)$ \\
\hline$S(2)$ & $11328(1)$ & $7001(1)$ & $5167(1)$ & $20(1)$ \\
\hline $\mathrm{O}(5)$ & $11441(2)$ & $6190(2)$ & $5136(1)$ & $38(1)$ \\
\hline $\mathrm{O}(6)$ & $11975(2)$ & $7444(2)$ & $4749(1)$ & $53(1)$ \\
\hline$C(110)$ & 12021(3) & $7259(3)$ & $5885(2)$ & $32(1)$ \\
\hline $\mathrm{F}(4)$ & $11413(2)$ & 6942(2) & $6318(1)$ & $62(1)$ \\
\hline $\mathrm{F}(5)$ & $13194(2)$ & $7031(2)$ & $5924(1)$ & $54(1)$ \\
\hline$F(6)$ & 11993(2) & $8014(2)$ & $5965(2)$ & $66(1)$ \\
\hline $\mathrm{N}(4)$ & $9083(2)$ & $7479(1)$ & 3991(1) & $13(1)$ \\
\hline
\end{tabular}




\begin{tabular}{|c|c|c|c|c|}
\hline $\mathrm{C}(41)$ & $9398(3)$ & $7772(2)$ & $3436(1)$ & $14(1)$ \\
\hline $\mathrm{C}(42)$ & $9453(3)$ & $8573(2)$ & $3346(2)$ & $17(1)$ \\
\hline$C(43)$ & $9757(3)$ & $8835(2)$ & $2794(2)$ & $24(1)$ \\
\hline $\mathrm{C}(44)$ & $10024(3)$ & $8330(2)$ & $2338(2)$ & $25(1)$ \\
\hline$C(45)$ & $9974(3)$ & $7547(2)$ & $2430(1)$ & $20(1)$ \\
\hline$C(46)$ & $9650(2)$ & $7253(2)$ & $2975(1)$ & $15(1)$ \\
\hline$C(421)$ & $9214(3)$ & $9130(2)$ & $3848(2)$ & $20(1)$ \\
\hline$C(422)$ & $8517(4)$ & $9853(2)$ & $3638(2)$ & $37(1)$ \\
\hline$C(423)$ & $10446(3)$ & $9344(2)$ & $4169(2)$ & $33(1)$ \\
\hline $\mathrm{C}(461)$ & $9621(3)$ & $6388(2)$ & $3086(2)$ & $19(1)$ \\
\hline$C(462)$ & $10915(3)$ & $6114(2)$ & $3298(2)$ & $30(1)$ \\
\hline$C(463)$ & $9153(3)$ & $5925(2)$ & $2552(2)$ & $26(1)$ \\
\hline $\mathrm{N}(5)$ & $7604(2)$ & $7850(1)$ & $5416(1)$ & $14(1)$ \\
\hline $\mathrm{C}(51)$ & $8193(3)$ & $7919(2)$ & $6008(1)$ & $14(1)$ \\
\hline$C(52)$ & $8842(3)$ & $8594(2)$ & $6141(2)$ & $19(1)$ \\
\hline$C(53)$ & $9371(3)$ & $8682(2)$ & $6703(2)$ & $25(1)$ \\
\hline$C(54)$ & $9238(3)$ & $8115(2)$ & $7132(2)$ & $26(1)$ \\
\hline$C(55)$ & $8597(3)$ & $7445(2)$ & $6986(2)$ & $22(1)$ \\
\hline$C(56)$ & $8075(3)$ & $7336(2)$ & $6427(1)$ & $18(1)$ \\
\hline$C(57)$ & $8976(4)$ & $9207(2)$ & $5668(2)$ & $31(1)$ \\
\hline$C(58)$ & $7392(4)$ & $6601(2)$ & $6272(2)$ & $29(1)$ \\
\hline $\mathrm{N}(6)$ & $6723(2)$ & $6828(1)$ & 4493(1) & $13(1)$ \\
\hline $\mathrm{C}(61)$ & $6537(3)$ & $6131(2)$ & $4176(2)$ & $14(1)$ \\
\hline$C(62)$ & 7601(3) & $5674(2)$ & $4194(2)$ & $15(1)$ \\
\hline$C(63)$ & $7590(3)$ & $4988(2)$ & $3880(2)$ & $20(1)$ \\
\hline$C(64)$ & $6534(3)$ & $4768(2)$ & $3565(2)$ & $22(1)$ \\
\hline$C(65)$ & $5457(3)$ & $5210(2)$ & $3577(2)$ & $22(1)$ \\
\hline$C(66)$ & $5425(3)$ & $5908(2)$ & $3896(2)$ & $18(1)$ \\
\hline$C(67)$ & $8648(3)$ & $6003(2)$ & $4570(2)$ & $15(1)$ \\
\hline$C(68)$ & $4238(3)$ & $6351(2)$ & $3931(2)$ & $25(1)$ \\
\hline$C(101)$ & $6478(3)$ & $8068(2)$ & $5346(2)$ & $13(1)$ \\
\hline$C(102)$ & $5905(3)$ & $8118(2)$ & $4726(1)$ & $11(1)$ \\
\hline$C(103)$ & $6579(2)$ & $7572(2)$ & $4306(1)$ & $13(1)$ \\
\hline C(104) & $5701(3)$ & $8334(2)$ & $5851(2)$ & $20(1)$ \\
\hline$C(105)$ & $6391(3)$ & $7718(2)$ & $3644(1)$ & $15(1)$ \\
\hline$C(106)$ & $5998(3)$ & 8993(2) & $4557(2)$ & $14(1)$ \\
\hline
\end{tabular}




$\begin{array}{lrrrr}\mathrm{C}(107) & 4793(3) & 9419(2) & 4347(2) & 18(1) \\ \mathrm{C}(108) & 5182(3) & 10258(2) & 4248(2) & 29(1) \\ \mathrm{C}(109) & 3841(3) & 9424(2) & 4839(2) & 24(1) \\ \mathrm{C}(81) & 4250(3) & 9063(2) & 3778(2) & 17(1) \\ \mathrm{C}(82) & 3333(3) & 8501(2) & 3797(2) & 21(1) \\ \mathrm{C}(83) & 2870(3) & 8150(2) & 3279(2) & 23(1) \\ \mathrm{C}(84) & 3311(3) & 8347(2) & 2739(2) & 29(1) \\ \mathrm{C}(85) & 4215(3) & 8906(2) & 2713(2) & 30(1) \\ \mathrm{C}(86) & 4675(3) & 9265(2) & 3222(2) & 24(1)\end{array}$


Bond lengths $[\AA]$ and angles $\left[{ }^{\circ}\right]$ for $\mathbf{3 a "}$ (06187).

\begin{tabular}{|c|c|}
\hline- & \\
\hline Mo(1)-N(1) & $1.723(3)$ \\
\hline $\operatorname{Mo}(1)-N(3)$ & $1.992(3)$ \\
\hline $\mathrm{Mo}(1)-\mathrm{O}(1)$ & $2.127(2)$ \\
\hline $\operatorname{Mo}(1)-C(37)$ & $2.165(3)$ \\
\hline $\mathrm{Mo}(1)-\mathrm{C}(3)$ & $2.207(3)$ \\
\hline $\operatorname{Mo}(1)-N(2)$ & $2.239(3)$ \\
\hline $\mathrm{O}(1)-\mathrm{S}(1)$ & $1.487(2)$ \\
\hline $\mathrm{S}(1)-\mathrm{O}(3)$ & $1.422(3)$ \\
\hline $\mathrm{S}(1)-\mathrm{O}(2)$ & $1.425(3)$ \\
\hline$S(1)-C(10)$ & $1.824(4)$ \\
\hline $\mathrm{F}(1)-\mathrm{C}(10)$ & $1.307(5)$ \\
\hline $\mathrm{F}(2)-\mathrm{C}(10)$ & $1.330(4)$ \\
\hline $\mathrm{F}(3)-\mathrm{C}(10)$ & $1.339(4)$ \\
\hline $\mathrm{N}(1)-\mathrm{C}(11)$ & $1.403(4)$ \\
\hline $\mathrm{C}(11)-\mathrm{C}(12)$ & $1.404(4)$ \\
\hline$C(11)-C(16)$ & $1.421(4)$ \\
\hline$C(12)-C(13)$ & $1.383(4)$ \\
\hline$C(12)-C(121)$ & $1.522(4)$ \\
\hline$C(13)-C(14)$ & $1.386(5)$ \\
\hline$C(14)-C(15)$ & $1.389(5)$ \\
\hline$C(15)-C(16)$ & $1.380(5)$ \\
\hline$C(16)-C(161)$ & $1.523(4)$ \\
\hline $\mathrm{C}(121)-\mathrm{C}(122)$ & $1.526(5)$ \\
\hline C(121)-C(123) & $1.540(5)$ \\
\hline C(161)-C(162) & $1.522(4)$ \\
\hline C(161)-C(163) & $1.532(4)$ \\
\hline $\mathrm{N}(2)-\mathrm{C}(1)$ & $1.285(4)$ \\
\hline $\mathrm{N}(2)-\mathrm{C}(21)$ & $1.451(4)$ \\
\hline $\mathrm{C}(21)-\mathrm{C}(22)$ & $1.390(4)$ \\
\hline$C(21)-C(26)$ & $1.402(4)$ \\
\hline$C(22)-C(23)$ & $1.405(5)$ \\
\hline $\mathrm{C}(22)-\mathrm{C}(27)$ & $1.508(5)$ \\
\hline$C(23)-C(24)$ & $1.363(5)$ \\
\hline
\end{tabular}




\begin{tabular}{|c|c|}
\hline$C(24)-C(25)$ & $1.382(5)$ \\
\hline$C(25)-C(26)$ & $1.390(5)$ \\
\hline$C(26)-C(28)$ & $1.507(5)$ \\
\hline$N(3)-C(3)$ & $1.380(4)$ \\
\hline $\mathrm{N}(3)-\mathrm{C}(31)$ & $1.412(4)$ \\
\hline $\mathrm{C}(31)-\mathrm{C}(32)$ & $1.388(4)$ \\
\hline$C(31)-C(36)$ & $1.398(4)$ \\
\hline$C(32)-C(33)$ & $1.397(4)$ \\
\hline$C(32)-C(37)$ & $1.491(5)$ \\
\hline$C(33)-C(34)$ & $1.385(5)$ \\
\hline$C(34)-C(35)$ & $1.377(5)$ \\
\hline$C(35)-C(36)$ & $1.405(4)$ \\
\hline$C(36)-C(38)$ & $1.496(4)$ \\
\hline$C(1)-C(4)$ & $1.496(5)$ \\
\hline$C(1)-C(2)$ & $1.505(5)$ \\
\hline$C(2)-C(3)$ & $1.541(4)$ \\
\hline$C(2)-C(6)$ & $1.580(4)$ \\
\hline$C(3)-C(5)$ & $1.510(4)$ \\
\hline$C(6)-C(7)$ & $1.557(4)$ \\
\hline $\mathrm{C}(7)-\mathrm{C}(71)$ & $1.521(5)$ \\
\hline$C(7)-C(9)$ & $1.525(4)$ \\
\hline$C(7)-C(8)$ & $1.553(4)$ \\
\hline $\mathrm{C}(71)-\mathrm{C}(72)$ & $1.384(5)$ \\
\hline $\mathrm{C}(71)-\mathrm{C}(76)$ & $1.399(5)$ \\
\hline $\mathrm{C}(72)-\mathrm{C}(73)$ & $1.376(5)$ \\
\hline $\mathrm{C}(73)-\mathrm{C}(74)$ & $1.383(6)$ \\
\hline$C(74)-C(75)$ & $1.377(5)$ \\
\hline$C(75)-C(76)$ & $1.391(5)$ \\
\hline $\mathrm{Mo}(2)-\mathrm{N}(4)$ & $1.727(2)$ \\
\hline $\mathrm{Mo}(2)-\mathrm{N}(6)$ & $1.998(3)$ \\
\hline $\mathrm{Mo}(2)-\mathrm{O}(4)$ & $2.127(2)$ \\
\hline $\operatorname{Mo}(2)-C(67)$ & $2.159(3)$ \\
\hline $\operatorname{Mo}(2)-C(103)$ & $2.206(3)$ \\
\hline $\mathrm{Mo}(2)-\mathrm{N}(5)$ & $2.239(3)$ \\
\hline $\mathrm{O}(4)-\mathrm{S}(2)$ & $1.485(2)$ \\
\hline $\mathrm{S}(2)-\mathrm{O}(5)$ & $1.418(3)$ \\
\hline
\end{tabular}




\begin{tabular}{|c|c|}
\hline $\mathrm{S}(2)-\mathrm{O}(6)$ & $1.421(3)$ \\
\hline $\mathrm{S}(2)-\mathrm{C}(110)$ & $1.816(4)$ \\
\hline $\mathrm{C}(110)-\mathrm{F}(4)$ & $1.315(5)$ \\
\hline $\mathrm{C}(110)-\mathrm{F}(5)$ & $1.322(4)$ \\
\hline$C(110)-F(6)$ & $1.328(4)$ \\
\hline $\mathrm{N}(4)-\mathrm{C}(41)$ & $1.403(4)$ \\
\hline$C(41)-C(42)$ & $1.410(4)$ \\
\hline$C(41)-C(46)$ & $1.412(4)$ \\
\hline$C(42)-C(43)$ & $1.378(5)$ \\
\hline$C(42)-C(421)$ & $1.520(5)$ \\
\hline$C(43)-C(44)$ & $1.390(5)$ \\
\hline$C(44)-C(45)$ & $1.379(5)$ \\
\hline$C(45)-C(46)$ & $1.387(4)$ \\
\hline$C(46)-C(461)$ & $1.526(5)$ \\
\hline $\mathrm{C}(421)-\mathrm{C}(422)$ & $1.532(5)$ \\
\hline $\mathrm{C}(421)-\mathrm{C}(423)$ & $1.532(5)$ \\
\hline$C(461)-C(463)$ & $1.521(5)$ \\
\hline$C(461)-C(462)$ & $1.531(5)$ \\
\hline $\mathrm{N}(5)-\mathrm{C}(101)$ & $1.274(4)$ \\
\hline $\mathrm{N}(5)-\mathrm{C}(51)$ & $1.463(4)$ \\
\hline$C(51)-C(52)$ & $1.394(4)$ \\
\hline$C(51)-C(56)$ & $1.395(4)$ \\
\hline$C(52)-C(53)$ & $1.379(5)$ \\
\hline$C(52)-C(57)$ & $1.519(5)$ \\
\hline$C(53)-C(54)$ & $1.393(5)$ \\
\hline$C(54)-C(55)$ & $1.388(5)$ \\
\hline$C(55)-C(56)$ & $1.377(4)$ \\
\hline$C(56)-C(58)$ & $1.510(4)$ \\
\hline $\mathrm{N}(6)-\mathrm{C}(103)$ & $1.369(4)$ \\
\hline $\mathrm{N}(6)-\mathrm{C}(61)$ & $1.419(4)$ \\
\hline$C(61)-C(66)$ & $1.388(4)$ \\
\hline$C(61)-C(62)$ & $1.393(4)$ \\
\hline$C(62)-C(63)$ & $1.388(4)$ \\
\hline$C(62)-C(67)$ & $1.500(4)$ \\
\hline$C(63)-C(64)$ & $1.373(5)$ \\
\hline$C(64)-C(65)$ & $1.392(5)$ \\
\hline
\end{tabular}




\begin{tabular}{|c|c|}
\hline$C(65)-C(66)$ & $1.412(5)$ \\
\hline$C(66)-C(68)$ & $1.497(4)$ \\
\hline$C(101)-C(104)$ & $1.509(4)$ \\
\hline$C(101)-C(102)$ & $1.510(4)$ \\
\hline$C(102)-C(103)$ & $1.541(4)$ \\
\hline$C(102)-C(106)$ & $1.574(4)$ \\
\hline$C(103)-C(105)$ & $1.522(4)$ \\
\hline$C(106)-C(107)$ & $1.551(4)$ \\
\hline $\mathrm{C}(107)-\mathrm{C}(81)$ & $1.525(5)$ \\
\hline$C(107)-C(109)$ & $1.536(5)$ \\
\hline$C(107)-C(108)$ & $1.538(4)$ \\
\hline $\mathrm{C}(81)-\mathrm{C}(82)$ & $1.391(5)$ \\
\hline$C(81)-C(86)$ & $1.394(5)$ \\
\hline $\mathrm{C}(82)-\mathrm{C}(83)$ & $1.395(5)$ \\
\hline$C(83)-C(84)$ & $1.366(5)$ \\
\hline$C(84)-C(85)$ & $1.379(5)$ \\
\hline$C(85)-C(86)$ & $1.383(5)$ \\
\hline $\mathrm{N}(1)-\mathrm{Mo}(1)-\mathrm{N}(3)$ & 109.98(11) \\
\hline $\mathrm{N}(1)-\mathrm{Mo}(1)-\mathrm{O}(1)$ & $99.43(10)$ \\
\hline $\mathrm{N}(3)-\mathrm{Mo}(1)-\mathrm{O}(1)$ & $148.48(10)$ \\
\hline $\mathrm{N}(1)-\mathrm{Mo}(1)-\mathrm{C}(37)$ & $97.24(13)$ \\
\hline $\mathrm{N}(3)-\mathrm{Mo}(1)-\mathrm{C}(37)$ & $74.17(12)$ \\
\hline $\mathrm{O}(1)-\mathrm{Mo}(1)-\mathrm{C}(37)$ & $91.50(11)$ \\
\hline $\mathrm{N}(1)-\mathrm{Mo}(1)-\mathrm{C}(3)$ & $90.70(11)$ \\
\hline $\mathrm{N}(3)-\mathrm{Mo}(1)-\mathrm{C}(3)$ & $37.94(11)$ \\
\hline $\mathrm{O}(1)-\mathrm{Mo}(1)-\mathrm{C}(3)$ & $155.97(11)$ \\
\hline $\mathrm{C}(37)-\mathrm{Mo}(1)-\mathrm{C}(3)$ & $108.89(13)$ \\
\hline $\mathrm{N}(1)-\mathrm{Mo}(1)-\mathrm{N}(2)$ & $136.09(10)$ \\
\hline $\mathrm{N}(3)-\mathrm{Mo}(1)-\mathrm{N}(2)$ & $85.10(10)$ \\
\hline $\mathrm{O}(1)-\mathrm{Mo}(1)-\mathrm{N}(2)$ & $81.32(9)$ \\
\hline $\mathrm{C}(37)-\mathrm{Mo}(1)-\mathrm{N}(2)$ & $126.67(12)$ \\
\hline $\mathrm{C}(3)-\mathrm{Mo}(1)-\mathrm{N}(2)$ & $76.35(10)$ \\
\hline $\mathrm{S}(1)-\mathrm{O}(1)-\mathrm{Mo}(1)$ & $130.81(14)$ \\
\hline $\mathrm{O}(3)-\mathrm{S}(1)-\mathrm{O}(2)$ & $117.28(18)$ \\
\hline $\mathrm{O}(3)-\mathrm{S}(1)-\mathrm{O}(1)$ & $113.49(15)$ \\
\hline
\end{tabular}




\begin{tabular}{|c|c|}
\hline $\mathrm{O}(2)-\mathrm{S}(1)-\mathrm{O}(1)$ & $4(15)$ \\
\hline $\mathrm{O}(3)-\mathrm{S}(1)-\mathrm{C}(10)$ & $105.1(2)$ \\
\hline $\mathrm{O}(2)-\mathrm{S}(1)-\mathrm{C}(10)$ & $105.11(18)$ \\
\hline $\mathrm{O}(1)-\mathrm{S}(1)-\mathrm{C}(10)$ & $99.04(16)$ \\
\hline $\mathrm{C}(11)-\mathrm{N}(1)-\mathrm{Mo}(1)$ & $168.3(2)$ \\
\hline$F(1)-C(10)-F(2)$ & $108.7(4)$ \\
\hline$F(1)-C(10)-F(3)$ & $109.1(3)$ \\
\hline $\mathrm{F}(2)-\mathrm{C}(10)-\mathrm{F}(3)$ & $107.4(3)$ \\
\hline$F(1)-C(10)-S(1)$ & $111.4(3)$ \\
\hline $\mathrm{F}(2)-\mathrm{C}(10)-\mathrm{S}(1)$ & $111.0(3)$ \\
\hline$F(3)-C(10)-S(1)$ & $109.1(3)$ \\
\hline $\mathrm{N}(1)-\mathrm{C}(11)-\mathrm{C}(12)$ & $119.5(3)$ \\
\hline $\mathrm{N}(1)-\mathrm{C}(11)-\mathrm{C}(16)$ & $119.0(3)$ \\
\hline$C(12)-C(11)-C(16)$ & $121.4(3)$ \\
\hline$C(13)-C(12)-C(11)$ & $118.6(3)$ \\
\hline$C(13)-C(12)-C(121)$ & $121.6(3)$ \\
\hline$C(11)-C(12)-C(121)$ & $119.8(3)$ \\
\hline$C(12)-C(13)-C(14)$ & $120.9(3)$ \\
\hline$C(13)-C(14)-C(15)$ & $119.8(3)$ \\
\hline$C(16)-C(15)-C(14)$ & $121.8(3)$ \\
\hline$C(15)-C(16)-C(11)$ & $117.4(3)$ \\
\hline$C(15)-C(16)-C(161)$ & $121.9(3)$ \\
\hline$C(11)-C(16)-C(161)$ & $120.7(3)$ \\
\hline$C(12)-C(121)-C(122)$ & $109.8(3)$ \\
\hline$C(12)-C(121)-C(123)$ & $112.3(3)$ \\
\hline $\mathrm{C}(122)-\mathrm{C}(121)-\mathrm{C}(123)$ & $110.5(3)$ \\
\hline$C(162)-C(161)-C(16)$ & $113.0(3)$ \\
\hline$C(162)-C(161)-C(163)$ & $110.5(3)$ \\
\hline$C(16)-C(161)-C(163)$ & $110.3(3)$ \\
\hline $\mathrm{C}(1)-\mathrm{N}(2)-\mathrm{C}(21)$ & $118.0(3)$ \\
\hline $\mathrm{C}(1)-\mathrm{N}(2)-\mathrm{Mo}(1)$ & $117.0(2)$ \\
\hline $\mathrm{C}(21)-\mathrm{N}(2)-\mathrm{Mo}(1)$ & $124.45(18)$ \\
\hline$C(22)-C(21)-C(26)$ & $121.9(3)$ \\
\hline$C(22)-C(21)-N(2)$ & $120.0(3)$ \\
\hline $\mathrm{C}(26)-\mathrm{C}(21)-\mathrm{N}(2)$ & $118.1(3)$ \\
\hline$C(21)-C(22)-C(23)$ & $117.8(3)$ \\
\hline
\end{tabular}




\begin{tabular}{|c|c|}
\hline $\mathrm{C}(21)-\mathrm{C}(22)-\mathrm{C}(27)$ & $122.1(3)$ \\
\hline $\mathrm{C}(23)-\mathrm{C}(22)-\mathrm{C}(27)$ & $120.1(3)$ \\
\hline $\mathrm{C}(24)-\mathrm{C}(23)-\mathrm{C}(22)$ & $121.1(3)$ \\
\hline $\mathrm{C}(23)-\mathrm{C}(24)-\mathrm{C}(25)$ & $120.4(3)$ \\
\hline $\mathrm{C}(24)-\mathrm{C}(25)-\mathrm{C}(26)$ & $120.9(3)$ \\
\hline$C(25)-C(26)-C(21)$ & $118.0(3)$ \\
\hline $\mathrm{C}(25)-\mathrm{C}(26)-\mathrm{C}(28)$ & $121.0(3)$ \\
\hline $\mathrm{C}(21)-\mathrm{C}(26)-\mathrm{C}(28)$ & $121.0(3)$ \\
\hline $\mathrm{C}(3)-\mathrm{N}(3)-\mathrm{C}(31)$ & $129.3(3)$ \\
\hline $\mathrm{C}(3)-\mathrm{N}(3)-\mathrm{Mo}(1)$ & $79.51(16)$ \\
\hline $\mathrm{C}(31)-\mathrm{N}(3)-\mathrm{Mo}(1)$ & $121.2(2)$ \\
\hline $\mathrm{C}(32)-\mathrm{C}(31)-\mathrm{C}(36)$ & $122.9(3)$ \\
\hline $\mathrm{C}(32)-\mathrm{C}(31)-\mathrm{N}(3)$ & $111.7(3)$ \\
\hline $\mathrm{C}(36)-\mathrm{C}(31)-\mathrm{N}(3)$ & $125.4(3)$ \\
\hline $\mathrm{C}(31)-\mathrm{C}(32)-\mathrm{C}(33)$ & $119.4(3)$ \\
\hline $\mathrm{C}(31)-\mathrm{C}(32)-\mathrm{C}(37)$ & $113.7(3)$ \\
\hline $\mathrm{C}(33)-\mathrm{C}(32)-\mathrm{C}(37)$ & $126.9(3)$ \\
\hline $\mathrm{C}(34)-\mathrm{C}(33)-\mathrm{C}(32)$ & $118.6(3)$ \\
\hline $\mathrm{C}(35)-\mathrm{C}(34)-\mathrm{C}(33)$ & $121.3(3)$ \\
\hline $\mathrm{C}(34)-\mathrm{C}(35)-\mathrm{C}(36)$ & $121.7(3)$ \\
\hline $\mathrm{C}(31)-\mathrm{C}(36)-\mathrm{C}(35)$ & $115.9(3)$ \\
\hline $\mathrm{C}(31)-\mathrm{C}(36)-\mathrm{C}(38)$ & $122.7(3)$ \\
\hline $\mathrm{C}(35)-\mathrm{C}(36)-\mathrm{C}(38)$ & $121.4(3)$ \\
\hline $\mathrm{C}(32)-\mathrm{C}(37)-\mathrm{Mo}(1)$ & $110.8(2)$ \\
\hline $\mathrm{N}(2)-\mathrm{C}(1)-\mathrm{C}(4)$ & $123.7(3)$ \\
\hline $\mathrm{N}(2)-\mathrm{C}(1)-\mathrm{C}(2)$ & $118.4(3)$ \\
\hline$C(4)-C(1)-C(2)$ & $117.9(3)$ \\
\hline$C(1)-C(2)-C(3)$ & $111.1(2)$ \\
\hline $\mathrm{C}(1)-\mathrm{C}(2)-\mathrm{C}(6)$ & $105.3(3)$ \\
\hline $\mathrm{C}(3)-\mathrm{C}(2)-\mathrm{C}(6)$ & $114.4(3)$ \\
\hline $\mathrm{N}(3)-\mathrm{C}(3)-\mathrm{C}(5)$ & $117.8(3)$ \\
\hline $\mathrm{N}(3)-\mathrm{C}(3)-\mathrm{C}(2)$ & $116.2(3)$ \\
\hline $\mathrm{C}(5)-\mathrm{C}(3)-\mathrm{C}(2)$ & $117.9(3)$ \\
\hline $\mathrm{N}(3)-\mathrm{C}(3)-\mathrm{Mo}(1)$ & $62.55(16)$ \\
\hline $\mathrm{C}(5)-\mathrm{C}(3)-\mathrm{Mo}(1)$ & $118.23(19)$ \\
\hline $\mathrm{C}(2)-\mathrm{C}(3)-\mathrm{Mo}(1)$ & $112.25(19)$ \\
\hline
\end{tabular}




\begin{tabular}{|c|c|}
\hline$C(7)-C(6)-C(2)$ & $117.7(3)$ \\
\hline $\mathrm{C}(71)-\mathrm{C}(7)-\mathrm{C}(9)$ & $111.8(3)$ \\
\hline$C(71)-C(7)-C(8)$ & $110.9(3)$ \\
\hline$C(9)-C(7)-C(8)$ & $106.9(3)$ \\
\hline$C(71)-C(7)-C(6)$ & $110.3(2)$ \\
\hline$C(9)-C(7)-C(6)$ & $111.5(3)$ \\
\hline $\mathrm{C}(8)-\mathrm{C}(7)-\mathrm{C}(6)$ & $105.3(2)$ \\
\hline$C(72)-C(71)-C(76)$ & $117.0(3)$ \\
\hline $\mathrm{C}(72)-\mathrm{C}(71)-\mathrm{C}(7)$ & $121.9(3)$ \\
\hline $\mathrm{C}(76)-\mathrm{C}(71)-\mathrm{C}(7)$ & $121.0(3)$ \\
\hline $\mathrm{C}(73)-\mathrm{C}(72)-\mathrm{C}(71)$ & $122.4(4)$ \\
\hline $\mathrm{C}(72)-\mathrm{C}(73)-\mathrm{C}(74)$ & $120.1(4)$ \\
\hline $\mathrm{C}(75)-\mathrm{C}(74)-\mathrm{C}(73)$ & $119.0(4)$ \\
\hline$C(74)-C(75)-C(76)$ & $120.6(4)$ \\
\hline $\mathrm{C}(75)-\mathrm{C}(76)-\mathrm{C}(71)$ & $120.9(3)$ \\
\hline $\mathrm{N}(4)-\mathrm{Mo}(2)-\mathrm{N}(6)$ & $108.94(11)$ \\
\hline $\mathrm{N}(4)-\mathrm{Mo}(2)-\mathrm{O}(4)$ & $100.94(10)$ \\
\hline $\mathrm{N}(6)-\mathrm{Mo}(2)-\mathrm{O}(4)$ & $147.79(10)$ \\
\hline $\mathrm{N}(4)-\mathrm{Mo}(2)-\mathrm{C}(67)$ & $97.10(12)$ \\
\hline N(6)-Mo(2)-C(67) & $74.55(11)$ \\
\hline $\mathrm{O}(4)-\mathrm{Mo}(2)-\mathrm{C}(67)$ & $90.35(11)$ \\
\hline $\mathrm{N}(4)-\mathrm{Mo}(2)-\mathrm{C}(103)$ & $90.52(11)$ \\
\hline $\mathrm{N}(6)-\mathrm{Mo}(2)-\mathrm{C}(103)$ & $37.59(10)$ \\
\hline $\mathrm{O}(4)-\mathrm{Mo}(2)-\mathrm{C}(103)$ & $156.01(10)$ \\
\hline $\mathrm{C}(67)-\mathrm{Mo}(2)-\mathrm{C}(103)$ & $109.25(12)$ \\
\hline $\mathrm{N}(4)-\mathrm{Mo}(2)-\mathrm{N}(5)$ & $136.76(10)$ \\
\hline $\mathrm{N}(6)-\mathrm{Mo}(2)-\mathrm{N}(5)$ & $85.16(10)$ \\
\hline $\mathrm{O}(4)-\mathrm{Mo}(2)-\mathrm{N}(5)$ & $81.12(9)$ \\
\hline $\mathrm{C}(67)-\mathrm{Mo}(2)-\mathrm{N}(5)$ & $126.14(12)$ \\
\hline $\mathrm{C}(103)-\mathrm{Mo}(2)-\mathrm{N}(5)$ & $76.17(10)$ \\
\hline $\mathrm{S}(2)-\mathrm{O}(4)-\mathrm{Mo}(2)$ & $131.21(13)$ \\
\hline $\mathrm{O}(5)-\mathrm{S}(2)-\mathrm{O}(6)$ & $117.5(2)$ \\
\hline $\mathrm{O}(5)-\mathrm{S}(2)-\mathrm{O}(4)$ & $114.02(15)$ \\
\hline $\mathrm{O}(6)-\mathrm{S}(2)-\mathrm{O}(4)$ & $112.44(15)$ \\
\hline $\mathrm{O}(5)-\mathrm{S}(2)-\mathrm{C}(110)$ & $104.9(2)$ \\
\hline $\mathrm{O}(6)-\mathrm{S}(2)-\mathrm{C}(110)$ & $105.3(2)$ \\
\hline
\end{tabular}




$\begin{array}{ll}\mathrm{O}(4)-\mathrm{S}(2)-\mathrm{C}(110) & 100.33(15) \\ \mathrm{F}(4)-\mathrm{C}(110)-\mathrm{F}(5) & 108.9(4) \\ \mathrm{F}(4)-\mathrm{C}(110)-\mathrm{F}(6) & 107.4(4) \\ \mathrm{F}(5)-\mathrm{C}(110)-\mathrm{F}(6) & 108.4(3) \\ \mathrm{F}(4)-\mathrm{C}(110)-\mathrm{S}(2) & 111.0(3) \\ \mathrm{F}(5)-\mathrm{C}(110)-\mathrm{S}(2) & 110.2(3) \\ \mathrm{F}(6)-\mathrm{C}(110)-\mathrm{S}(2) & 110.8(3) \\ \mathrm{C}(41)-\mathrm{N}(4)-\mathrm{Mo}(2) & 168.7(2) \\ \mathrm{N}(4)-\mathrm{C}(41)-\mathrm{C}(42) & 120.0(3) \\ \mathrm{N}(4)-\mathrm{C}(41)-\mathrm{C}(46) & 118.9(3) \\ \mathrm{C}(42)-\mathrm{C}(41)-\mathrm{C}(46) & 121.1(3) \\ \mathrm{C}(43)-\mathrm{C}(42)-\mathrm{C}(41) & 118.1(3) \\ \mathrm{C}(43)-\mathrm{C}(42)-\mathrm{C}(421) & 120.9(3) \\ \mathrm{C}(41)-\mathrm{C}(42)-\mathrm{C}(421) & 121.0(3) \\ \mathrm{C}(42)-\mathrm{C}(43)-\mathrm{C}(44) & 121.4(3) \\ \mathrm{C}(45)-\mathrm{C}(44)-\mathrm{C}(43) & 120.2(3) \\ \mathrm{C}(44)-\mathrm{C}(45)-\mathrm{C}(46) & 120.7(3) \\ \mathrm{C}(45)-\mathrm{C}(46)-\mathrm{C}(41) & 118.5(3) \\ \mathrm{C}(45)-\mathrm{C}(46)-\mathrm{C}(461) & 121.2(3) \\ \mathrm{C}(41)-\mathrm{C}(46)-\mathrm{C}(461) & 120.3(3) \\ \mathrm{C}(42)-\mathrm{C}(421)-\mathrm{C}(422) & 112.9(3) \\ \mathrm{C}(42)-\mathrm{C}(421)-\mathrm{C}(423) & 109.9(3) \\ \mathrm{C}(422)-\mathrm{C}(421)-\mathrm{C}(423) & 110.6(3) \\ \mathrm{C}(463)-\mathrm{C}(461)-\mathrm{C}(46) & 113.6(3) \\ \mathrm{C}(463)-\mathrm{C}(461)-\mathrm{C}(462) & 110.7(3) \\ \mathrm{C}(46)-\mathrm{C}(461)-\mathrm{C}(462) & 109.5(3) \\ \mathrm{C}(101)-\mathrm{N}(5)-\mathrm{C}(51) & 117.9(3) \\ \mathrm{C}(101)-\mathrm{N}(5)-\mathrm{Mo}(2) & 117.0(2) \\ \mathrm{C}(51)-\mathrm{N}(5)-\mathrm{Mo}(2) & 124.46(18) \\ \mathrm{C}(52)-\mathrm{C}(51)-\mathrm{C}(56) & 121.6(3) \\ \mathrm{C}(52)-\mathrm{C}(51)-\mathrm{N}(5) & 117.6(3) \\ \mathrm{C}(56)-\mathrm{C}(51)-\mathrm{N}(5) & 120.8(3) \\ \mathrm{C}(53)-\mathrm{C}(52)-\mathrm{C}(51) & 118.6(3) \\ \mathrm{C}(52)-\mathrm{C}(53)-\mathrm{C}(54) & 120.7(3) \\ & \end{array}$




\begin{tabular}{|c|c|}
\hline$C(55)-($ & $119.5(3)$ \\
\hline$C(56)-C(55)-C(54)$ & $121.0(3)$ \\
\hline$C(55)-C(56)-C(51)$ & $118.5(3)$ \\
\hline$C(55)-C(56)-C(58)$ & $120.6(3)$ \\
\hline$C(51)-C(56)-C(58)$ & $121.0(3)$ \\
\hline$C(103)-N(6)-C(61)$ & $129.9(3)$ \\
\hline $\mathrm{C}(103)-\mathrm{N}(6)-\mathrm{Mo}(2)$ & $79.48(17)$ \\
\hline $\mathrm{C}(61)-\mathrm{N}(6)-\mathrm{Mo}(2)$ & $120.4(2)$ \\
\hline$C(66)-C(61)-C(62)$ & $123.1(3)$ \\
\hline$C(66)-C(61)-N(6)$ & $125.0(3)$ \\
\hline $\mathrm{C}(62)-\mathrm{C}(61)-\mathrm{N}(6)$ & $111.9(3)$ \\
\hline$C(63)-C(62)-C(61)$ & $119.0(3)$ \\
\hline$C(63)-C(62)-C(67)$ & $127.6(3)$ \\
\hline$C(61)-C(62)-C(67)$ & $113.5(3)$ \\
\hline$C(64)-C(63)-C(62)$ & 119.7(3) \\
\hline$C(63)-C(64)-C(65)$ & $120.6(3)$ \\
\hline$C(64)-C(65)-C(66)$ & $121.4(3)$ \\
\hline$C(61)-C(66)-C(65)$ & $115.9(3)$ \\
\hline$C(61)-C(66)-C(68)$ & $123.6(3)$ \\
\hline$C(65)-C(66)-C(68)$ & $120.4(3)$ \\
\hline $\mathrm{C}(62)-\mathrm{C}(67)-\mathrm{Mo}(2)$ & $110.5(2)$ \\
\hline $\mathrm{N}(5)-\mathrm{C}(101)-\mathrm{C}(104)$ & $123.2(3)$ \\
\hline $\mathrm{N}(5)-\mathrm{C}(101)-\mathrm{C}(102)$ & 119.1(3) \\
\hline$C(104)-C(101)-C(102)$ & $117.6(3)$ \\
\hline$C(101)-C(102)-C(103)$ & $110.4(2)$ \\
\hline$C(101)-C(102)-C(106)$ & $104.6(2)$ \\
\hline$C(103)-C(102)-C(106)$ & $114.4(3)$ \\
\hline$N(6)-C(103)-C(105)$ & $118.1(3)$ \\
\hline $\mathrm{N}(6)-\mathrm{C}(103)-\mathrm{C}(102)$ & $116.4(3)$ \\
\hline$C(105)-C(103)-C(102)$ & $116.8(3)$ \\
\hline $\mathrm{N}(6)-\mathrm{C}(103)-\mathrm{Mo}(2)$ & $62.93(16)$ \\
\hline $\mathrm{C}(105)-\mathrm{C}(103)-\mathrm{Mo}(2)$ & $118.88(19)$ \\
\hline $\mathrm{C}(102)-\mathrm{C}(103)-\mathrm{Mo}(2)$ & $112.75(19)$ \\
\hline$C(107)-C(106)-C(102)$ & $118.4(2)$ \\
\hline $\mathrm{C}(81)-\mathrm{C}(107)-\mathrm{C}(109)$ & $111.4(3)$ \\
\hline $\mathrm{C}(81)-\mathrm{C}(107)-\mathrm{C}(108)$ & 111.1(3) \\
\hline
\end{tabular}




$\begin{array}{ll}\mathrm{C}(109)-\mathrm{C}(107)-\mathrm{C}(108) & 106.9(3) \\ \mathrm{C}(81)-\mathrm{C}(107)-\mathrm{C}(106) & 110.8(2) \\ \mathrm{C}(109)-\mathrm{C}(107)-\mathrm{C}(106) & 110.7(3) \\ \mathrm{C}(108)-\mathrm{C}(107)-\mathrm{C}(106) & 105.6(3) \\ \mathrm{C}(82)-\mathrm{C}(81)-\mathrm{C}(86) & 117.4(3) \\ \mathrm{C}(82)-\mathrm{C}(81)-\mathrm{C}(107) & 120.7(3) \\ \mathrm{C}(86)-\mathrm{C}(81)-\mathrm{C}(107) & 121.8(3) \\ \mathrm{C}(81)-\mathrm{C}(82)-\mathrm{C}(83) & 120.9(3) \\ \mathrm{C}(84)-\mathrm{C}(83)-\mathrm{C}(82) & 120.8(3) \\ \mathrm{C}(83)-\mathrm{C}(84)-\mathrm{C}(85) & 118.9(4) \\ \mathrm{C}(84)-\mathrm{C}(85)-\mathrm{C}(86) & 121.0(4) \\ \mathrm{C}(85)-\mathrm{C}(86)-\mathrm{C}(81) & 120.9(3)\end{array}$


Anisotropic displacement parameters $\left(\AA^{2} \times 10^{3}\right)$ for 3a" (06187). The anisotropic displacement factor exponent takes the form: $-2{ }^{2}\left[h^{2} \mathrm{a}^{* 2} \mathrm{U}^{11}+\ldots+2 h k \mathrm{a}^{*} \mathrm{~b}^{*} \mathrm{U}^{12}\right]$.
$\mathrm{U}^{1}$
$\mathrm{U}^{22}$
$\mathrm{U}^{33}$
$\mathrm{U}^{23}$
$\mathrm{U}^{13}$
$\mathrm{U}^{12}$

$\begin{array}{lcccccc}- & & & & \\ \mathrm{Mo}(1) & 15(1) & 10(1) & 12(1) & 1(1) & -1(1) & -2(1) \\ \mathrm{O}(1) & 18(1) & 25(1) & 20(1) & 2(1) & 1(1) & 0(1) \\ \mathrm{S}(1) & 16(1) & 23(1) & 26(1) & 8(1) & -1(1) & -4(1) \\ \mathrm{O}(2) & 26(1) & 26(1) & 32(2) & -6(1) & 9(1) & -8(1) \\ \mathrm{O}(3) & 33(2) & 50(2) & 61(2) & 33(2) & -20(1) & -9(1) \\ \mathrm{F}(1) & 66(2) & 58(2) & 23(2) & 3(1) & 17(1) & -1(1) \\ \mathrm{F}(2) & 44(2) & 29(1) & 96(2) & -28(1) & 30(2) & -9(1) \\ \mathrm{F}(3) & 32(1) & 43(2) & 96(2) & -22(1) & 36(1) & -13(1) \\ \mathrm{N}(1) & 14(1) & 14(1) & 12(1) & -3(1) & 0(1) & -2(1) \\ \mathrm{C}(10) & 28(2) & 23(2) & 51(3) & -9(2) & 19(2) & 0(2) \\ \mathrm{C}(11) & 15(1) & 16(2) & 13(2) & 2(1) & 3(1) & 1(1) \\ \mathrm{C}(12) & 19(1) & 16(2) & 17(2) & -1(1) & -1(1) & -1(1) \\ \mathrm{C}(13) & 31(2) & 25(2) & 12(2) & -3(2) & 0(1) & -3(2) \\ \mathrm{C}(14) & 36(2) & 26(2) & 14(2) & 7(1) & -4(1) & 0(1) \\ \mathrm{C}(15) & 30(2) & 18(2) & 21(2) & 6(1) & -5(1) & -1(1) \\ \mathrm{C}(16) & 18(2) & 15(2) & 15(2) & 2(1) & -1(1) & -3(1) \\ \mathrm{C}(121) & 38(2) & 16(2) & 13(2) & -3(1) & -4(2) & 5(1) \\ \mathrm{C}(122) & 58(3) & 19(2) & 62(3) & 8(2) & 35(2) & -1(2) \\ \mathrm{C}(123) & 31(2) & 21(2) & 32(2) & -10(2) & 1(2) & -1(1) \\ \mathrm{C}(161) & 22(2) & 13(2) & 18(2) & 2(1) & -6(1) & -2(1) \\ \mathrm{C}(162) & 32(2) & 18(2) & 31(2) & 4(2) & -6(2) & -11(1) \\ \mathrm{C}(163) & 24(2) & 18(2) & 29(2) & -6(1) & -1(2) & -1(1) \\ \mathrm{N}(2) & 14(1) & 9(1) & 13(2) & 2(1) & 0(1) & 2(1) \\ \mathrm{C}(21) & 13(2) & 21(2) & 13(2) & -1(1) & -1(1) & -1(1) \\ \mathrm{C}(22) & 19(1) & 21(2) & 17(2) & 2(2) & 0(1) & -6(1) \\ \mathrm{C}(23) & 28(2) & 30(2) & 19(2) & 8(2) & 2(1) & -4(2) \\ \mathrm{C}(24) & 27(2) & 45(2) & 9(2) & 2(2) & 5(1) & 1(2) \\ \mathrm{C}(25) & 24(2) & 29(2) & 22(2) & -5(2) & 0(1) & 6(2) \\ \mathrm{C}(26) & 20(2) & 24(2) & 16(2) & 3(1) & -2(1) & 0(1)\end{array}$




\begin{tabular}{|c|c|c|c|c|c|c|}
\hline$C(27)$ & $43(2)$ & $20(2)$ & $23(2)$ & $7(2)$ & $3(2)$ & $2(2)$ \\
\hline$C(28)$ & $40(2)$ & $26(2)$ & $24(2)$ & $3(2)$ & $3(2)$ & $16(2)$ \\
\hline $\mathrm{N}(3)$ & $18(1)$ & $11(1)$ & $14(2)$ & $-1(1)$ & $3(1)$ & $1(1)$ \\
\hline $\mathrm{C}(31)$ & $19(2)$ & $10(2)$ & $16(2)$ & $1(1)$ & $-2(1)$ & $3(1)$ \\
\hline$C(32)$ & $20(2)$ & $11(2)$ & $18(2)$ & $2(1)$ & $-3(1)$ & $2(1)$ \\
\hline $\mathrm{C}(33)$ & $25(2)$ & $13(2)$ & $23(2)$ & $1(1)$ & $-6(1)$ & $-1(1)$ \\
\hline$C(34)$ & $32(2)$ & $12(2)$ & $23(2)$ & $-2(2)$ & $-5(1)$ & $2(1)$ \\
\hline$C(35)$ & $31(2)$ & $15(2)$ & $15(2)$ & $-3(1)$ & $4(1)$ & $6(1)$ \\
\hline$C(36)$ & $22(2)$ & $13(2)$ & $17(2)$ & $5(1)$ & $-1(1)$ & $2(1)$ \\
\hline $\mathrm{C}(37)$ & $18(2)$ & $13(2)$ & $19(2)$ & $2(1)$ & $1(1)$ & $-3(1)$ \\
\hline $\mathrm{C}(38)$ & $23(2)$ & $14(2)$ & $32(2)$ & $0(2)$ & $5(2)$ & $3(1)$ \\
\hline $\mathrm{C}(1)$ & $22(2)$ & $7(2)$ & $13(2)$ & $2(1)$ & $-3(1)$ & $5(1)$ \\
\hline$C(2)$ & 11(1) & 11(1) & $15(2)$ & $0(1)$ & $-3(1)$ & $-2(1)$ \\
\hline$C(3)$ & $15(1)$ & $13(1)$ & $13(2)$ & $-3(2)$ & $1(1)$ & $2(1)$ \\
\hline $\mathrm{C}(4)$ & $19(2)$ & $24(2)$ & $19(2)$ & $1(2)$ & $-3(1)$ & $-3(1)$ \\
\hline$C(5)$ & $21(2)$ & $11(2)$ & $16(2)$ & $-1(1)$ & $1(1)$ & $-3(1)$ \\
\hline$C(6)$ & $19(2)$ & $9(1)$ & $19(2)$ & $-2(1)$ & $2(1)$ & $-1(1)$ \\
\hline$C(7)$ & $19(2)$ & $9(2)$ & $19(2)$ & $-2(1)$ & $4(1)$ & $-4(1)$ \\
\hline $\mathrm{C}(8)$ & $28(2)$ & $12(2)$ & $37(2)$ & $-1(2)$ & $8(2)$ & $-3(1)$ \\
\hline $\mathrm{C}(9)$ & $23(2)$ & $22(2)$ & $18(2)$ & $-3(2)$ & $1(1)$ & $-7(1)$ \\
\hline $\mathrm{C}(71)$ & $22(2)$ & $14(2)$ & $17(2)$ & $-1(1)$ & $4(1)$ & $-9(1)$ \\
\hline $\mathrm{C}(72)$ & $18(2)$ & $22(2)$ & $19(2)$ & $0(1)$ & $-2(1)$ & $-5(1)$ \\
\hline$C(73)$ & $25(2)$ & $25(2)$ & $40(2)$ & $-11(2)$ & $12(2)$ & $-7(1)$ \\
\hline $\mathrm{C}(74)$ & $47(2)$ & $33(2)$ & $19(2)$ & $-9(2)$ & $16(2)$ & $-20(2)$ \\
\hline$C(75)$ & $40(2)$ & $31(2)$ & $23(2)$ & $5(2)$ & $-2(2)$ & $-22(2)$ \\
\hline$C(76)$ & $29(2)$ & $14(2)$ & $21(2)$ & $4(1)$ & $1(1)$ & $-7(1)$ \\
\hline $\operatorname{Mo}(2)$ & $14(1)$ & $9(1)$ & $12(1)$ & $0(1)$ & $1(1)$ & $1(1)$ \\
\hline $\mathrm{O}(4)$ & $16(1)$ & $23(1)$ & $17(1)$ & $-4(1)$ & $2(1)$ & $-2(1)$ \\
\hline$S(2)$ & $16(1)$ & $26(1)$ & $18(1)$ & $2(1)$ & $0(1)$ & $3(1)$ \\
\hline $\mathrm{O}(5)$ & $29(2)$ & $27(2)$ & $57(2)$ & $-12(1)$ & $-15(1)$ & $10(1)$ \\
\hline $\mathrm{O}(6)$ & $28(1)$ & $87(3)$ & $45(2)$ & $32(2)$ & $16(1)$ & $12(1)$ \\
\hline$C(110)$ & $22(2)$ & $39(2)$ & $35(2)$ & $-8(2)$ & $-11(1)$ & $4(2)$ \\
\hline $\mathrm{F}(4)$ & $63(2)$ & $106(2)$ & $16(2)$ & $11(2)$ & $-8(1)$ & $12(2)$ \\
\hline $\mathrm{F}(5)$ & $31(1)$ & $64(2)$ & $66(2)$ & $-19(1)$ & $-23(1)$ & $15(1)$ \\
\hline $\mathrm{F}(6)$ & $43(2)$ & $44(2)$ & $110(3)$ & $-46(2)$ & $-23(2)$ & $6(1)$ \\
\hline $\mathrm{N}(4)$ & $16(1)$ & $9(1)$ & $14(1)$ & $0(1)$ & $2(1)$ & $2(1)$ \\
\hline
\end{tabular}




\begin{tabular}{|c|c|c|c|c|c|c|}
\hline$C(41)$ & $13(1)$ & $15(2)$ & $15(2)$ & $1(1)$ & $-1(1)$ & $0(1)$ \\
\hline $\mathrm{C}(42)$ & $16(2)$ & $15(2)$ & $21(2)$ & $1(1)$ & $1(1)$ & $3(1)$ \\
\hline$C(43)$ & $29(2)$ & $18(2)$ & $25(2)$ & $7(2)$ & $4(2)$ & $0(1)$ \\
\hline $\mathrm{C}(44)$ & $28(2)$ & $30(2)$ & $17(2)$ & $6(2)$ & $2(1)$ & $-4(2)$ \\
\hline$C(45)$ & $25(2)$ & $22(2)$ & $13(2)$ & $-2(2)$ & $2(1)$ & $-2(1)$ \\
\hline$C(46)$ & $15(1)$ & $16(1)$ & $12(2)$ & $-1(1)$ & $0(1)$ & $0(1)$ \\
\hline$C(421)$ & $21(2)$ & $16(2)$ & $25(2)$ & $0(1)$ & $9(1)$ & $-1(1)$ \\
\hline $\mathrm{C}(422)$ & $49(3)$ & $16(2)$ & $47(3)$ & $9(2)$ & $27(2)$ & $10(2)$ \\
\hline $\mathrm{C}(423)$ & $31(2)$ & $32(2)$ & $36(3)$ & $-17(2)$ & $11(2)$ & $-11(2)$ \\
\hline$C(461)$ & $22(2)$ & $14(2)$ & $21(2)$ & $-4(1)$ & $2(1)$ & $-1(1)$ \\
\hline $\mathrm{C}(462)$ & $36(2)$ & $18(2)$ & $37(2)$ & $5(2)$ & $-8(2)$ & $4(2)$ \\
\hline$C(463)$ & $31(2)$ & $22(2)$ & $26(2)$ & $-6(2)$ & $1(2)$ & $-1(1)$ \\
\hline $\mathrm{N}(5)$ & $16(1)$ & 11(1) & $14(2)$ & $2(1)$ & $2(1)$ & $-3(1)$ \\
\hline$C(51)$ & $14(1)$ & $17(1)$ & $13(2)$ & $-3(1)$ & $1(1)$ & $4(1)$ \\
\hline$C(52)$ & $23(2)$ & $18(2)$ & $17(2)$ & $-4(1)$ & $-1(1)$ & $-2(1)$ \\
\hline$C(53)$ & $29(2)$ & $23(2)$ & $22(2)$ & $-7(2)$ & $-6(1)$ & $-5(1)$ \\
\hline$C(54)$ & $32(2)$ & $30(2)$ & $16(2)$ & $-5(2)$ & $-9(2)$ & $2(2)$ \\
\hline$C(55)$ & $27(2)$ & $24(2)$ & $15(2)$ & $3(1)$ & $1(1)$ & $3(1)$ \\
\hline$C(56)$ & $24(2)$ & $17(2)$ & $14(2)$ & $-1(1)$ & $1(1)$ & $2(1)$ \\
\hline $\mathrm{C}(57)$ & $41(2)$ & $22(2)$ & $30(2)$ & $4(2)$ & $-8(2)$ & $-17(2)$ \\
\hline$C(58)$ & $49(2)$ & $19(2)$ & $20(2)$ & $3(2)$ & $-3(2)$ & $-8(2)$ \\
\hline $\mathrm{N}(6)$ & $14(1)$ & $9(1)$ & $15(2)$ & $1(1)$ & $-1(1)$ & $1(1)$ \\
\hline$C(61)$ & $22(2)$ & $11(2)$ & $11(2)$ & $2(1)$ & $2(1)$ & $-2(1)$ \\
\hline$C(62)$ & $20(2)$ & $12(2)$ & $12(2)$ & $2(1)$ & $2(1)$ & $-2(1)$ \\
\hline$C(63)$ & $25(2)$ & $11(2)$ & $23(2)$ & $0(1)$ & $5(1)$ & $2(1)$ \\
\hline$C(64)$ & $32(2)$ & $14(2)$ & $21(2)$ & $-7(1)$ & $3(2)$ & $-2(1)$ \\
\hline$C(65)$ & $27(2)$ & $18(2)$ & $20(2)$ & $-2(1)$ & $-2(1)$ & $-8(1)$ \\
\hline$C(66)$ & $20(2)$ & $15(2)$ & $20(2)$ & $3(1)$ & $1(1)$ & $-3(1)$ \\
\hline$C(67)$ & $17(2)$ & $13(2)$ & $15(2)$ & $2(1)$ & $-1(1)$ & $3(1)$ \\
\hline$C(68)$ & $21(2)$ & $20(2)$ & $34(2)$ & $2(2)$ & $-2(2)$ & $-6(1)$ \\
\hline$C(101)$ & $14(2)$ & $10(2)$ & $15(2)$ & $-1(1)$ & $2(1)$ & $-4(1)$ \\
\hline $\mathrm{C}(102)$ & $10(1)$ & $10(1)$ & $13(2)$ & $1(1)$ & $0(1)$ & $0(1)$ \\
\hline$C(103)$ & $13(1)$ & $12(1)$ & $13(1)$ & $-2(1)$ & $3(1)$ & $-6(1)$ \\
\hline$C(104)$ & $17(2)$ & $24(2)$ & $18(2)$ & $-1(1)$ & $2(1)$ & $5(1)$ \\
\hline$C(105)$ & $16(1)$ & $14(2)$ & $16(2)$ & $1(1)$ & $0(1)$ & $3(1)$ \\
\hline$C(106)$ & $18(1)$ & $13(2)$ & $13(2)$ & $-2(1)$ & $-3(1)$ & $0(1)$ \\
\hline
\end{tabular}




$\begin{array}{lllllll}\mathrm{C}(107) & 17(2) & 13(2) & 23(2) & -1(1) & -2(1) & 6(1) \\ \mathrm{C}(108) & 40(2) & 14(2) & 32(2) & 0(2) & -9(2) & 6(2) \\ \mathrm{C}(109) & 24(2) & 28(2) & 22(2) & -8(2) & -1(1) & 12(1) \\ \mathrm{C}(81) & 21(2) & 14(2) & 17(2) & 1(1) & -1(1) & 7(1) \\ \mathrm{C}(82) & 18(2) & 23(2) & 23(2) & 3(2) & 4(1) & 6(1) \\ \mathrm{C}(83) & 22(2) & 25(2) & 20(2) & -4(2) & -1(1) & -1(1) \\ \mathrm{C}(84) & 30(2) & 36(2) & 21(2) & -5(2) & -9(2) & 6(2) \\ \mathrm{C}(85) & 44(2) & 33(2) & 13(2) & 7(2) & 4(2) & 6(2) \\ \mathrm{C}(86) & 29(2) & 19(2) & 24(2) & 7(2) & 4(1) & 3(1)\end{array}$


Hydrogen coordinates $\left(\times 10^{4}\right)$ and isotropic displacement parameters $\left(\AA^{2} \times 10^{3}\right)$ for 3a" (06187).

\begin{tabular}{|c|c|c|c|c|}
\hline & $\mathrm{x}$ & $\mathrm{y}$ & $\mathrm{z}$ & $\mathrm{U}(\mathrm{eq})$ \\
\hline \multicolumn{5}{|l|}{-} \\
\hline $\mathrm{H}(13)$ & 5639 & 2505 & 2884 & 27 \\
\hline $\mathrm{H}(14)$ & 5876 & 1179 & 2973 & 30 \\
\hline $\mathrm{H}(15)$ & 5404 & 383 & 2168 & 27 \\
\hline $\mathrm{H}(121)$ & 4460 & 3483 & 1568 & 27 \\
\hline $\mathrm{H}(12 \mathrm{~A})$ & 6747 & 3607 & 2248 & 68 \\
\hline $\mathrm{H}(12 \mathrm{~B})$ & 6162 & 4288 & 1852 & 68 \\
\hline $\mathrm{H}(12 \mathrm{C})$ & 6629 & 3519 & 1542 & 68 \\
\hline $\mathrm{H}(12 \mathrm{D})$ & 3447 & 3511 & 2497 & 42 \\
\hline $\mathrm{H}(12 \mathrm{E})$ & 4136 & 4306 & 2371 & 42 \\
\hline $\mathrm{H}(12 \mathrm{~F})$ & 4752 & 3701 & 2827 & 42 \\
\hline $\mathrm{H}(161)$ & 3988 & 968 & 789 & 21 \\
\hline $\mathrm{H}(16 \mathrm{~A})$ & 4728 & -386 & 1419 & 41 \\
\hline $\mathrm{H}(16 \mathrm{~B})$ & 3772 & -341 & 860 & 41 \\
\hline $\mathrm{H}(16 \mathrm{C})$ & 3393 & 4 & 1486 & 41 \\
\hline $\mathrm{H}(16 \mathrm{D})$ & 6075 & 1091 & 549 & 35 \\
\hline $\mathrm{H}(16 \mathrm{E})$ & 5600 & 255 & 352 & 35 \\
\hline $\mathrm{H}(16 \mathrm{~F})$ & 6447 & 357 & 944 & 35 \\
\hline $\mathrm{H}(23)$ & 3601 & 2949 & -2226 & 31 \\
\hline $\mathrm{H}(24)$ & 4550 & 1828 & -2509 & 32 \\
\hline $\mathrm{H}(25)$ & 4621 & 771 & -1880 & 30 \\
\hline $\mathrm{H}(27 \mathrm{~A})$ & 3243 & 3666 & -1008 & 43 \\
\hline $\mathrm{H}(27 \mathrm{~B})$ & 1925 & 3258 & -931 & 43 \\
\hline $\mathrm{H}(27 \mathrm{C})$ & 2257 & 3628 & -1556 & 43 \\
\hline $\mathrm{H}(28 \mathrm{~A})$ & 4326 & 189 & -947 & 45 \\
\hline $\mathrm{H}(28 \mathrm{~B})$ & 2939 & 377 & -757 & 45 \\
\hline $\mathrm{H}(28 \mathrm{C})$ & 4101 & 738 & -392 & 45 \\
\hline $\mathrm{H}(33)$ & 3477 & 5171 & 1138 & 24 \\
\hline $\mathrm{H}(34)$ & 1729 & 5489 & 1673 & 27 \\
\hline
\end{tabular}




\begin{tabular}{|c|c|c|c|}
\hline $\mathrm{H}(35)$ & -27 & 4728 & 1630 \\
\hline $\mathrm{H}(37 \mathrm{~A})$ & 4661(19) & $3992(18)$ & $584(13)$ \\
\hline $\mathrm{H}(37 \mathrm{~B})$ & $3580(30)$ & 3969(19) & $37(9)$ \\
\hline $\mathrm{H}(38 \mathrm{~A})$ & -550 & 3144 & 662 \\
\hline $\mathrm{H}(38 \mathrm{~B})$ & -1249 & 3757 & 1061 \\
\hline $\mathrm{H}(38 \mathrm{C})$ & -542 & 3043 & 1367 \\
\hline $\mathrm{H}(2)$ & 211 & 1829 & 243 \\
\hline $\mathrm{H}(4 \mathrm{~A})$ & -14 & 1853 & -887 \\
\hline $\mathrm{H}(4 \mathrm{~B})$ & 479 & 989 & -818 \\
\hline $\mathrm{H}(4 \mathrm{C})$ & 1159 & 1574 & -1248 \\
\hline $\mathrm{H}(5 \mathrm{~A})$ & 2172 & 2500 & 1560 \\
\hline $\mathrm{H}(5 \mathrm{~B})$ & 2147 & 1595 & 1434 \\
\hline $\mathrm{H}(5 \mathrm{C})$ & 872 & 2069 & 1445 \\
\hline $\mathrm{H}(6 \mathrm{~A})$ & 1569 & 545 & 57 \\
\hline $\mathrm{H}(6 \mathrm{~B})$ & 1912 & 766 & 728 \\
\hline $\mathrm{H}(8 \mathrm{~A})$ & 1206 & -505 & 969 \\
\hline $\mathrm{H}(8 \mathrm{~B})$ & 739 & -675 & 300 \\
\hline $\mathrm{H}(8 \mathrm{C})$ & -195 & -778 & 831 \\
\hline $\mathrm{H}(9 \mathrm{~A})$ & -1679 & 113 & 277 \\
\hline $\mathrm{H}(9 \mathrm{~B})$ & -667 & 150 & -226 \\
\hline $\mathrm{H}(9 \mathrm{C})$ & -1191 & 927 & 51 \\
\hline $\mathrm{H}(72)$ & -1712 & 1387 & 865 \\
\hline $\mathrm{H}(73)$ & -2287 & 1932 & 1748 \\
\hline $\mathrm{H}(74)$ & -1269 & 1581 & 2640 \\
\hline $\mathrm{H}(75)$ & 377 & 711 & 2625 \\
\hline $\mathrm{H}(76)$ & 908 & 127 & 1739 \\
\hline $\mathrm{H}(43)$ & 9785 & 9373 & 2723 \\
\hline $\mathrm{H}(44)$ & 10242 & 8524 & 1962 \\
\hline $\mathrm{H}(45)$ & 10164 & 7206 & 2117 \\
\hline $\mathrm{H}(421)$ & 8687 & 8861 & 4139 \\
\hline $\mathrm{H}(42 \mathrm{~A})$ & 8297 & 10161 & 3983 \\
\hline $\mathrm{H}(42 \mathrm{~B})$ & 7757 & 9705 & 3415 \\
\hline $\mathrm{H}(42 \mathrm{C})$ & 9049 & 10157 & 3384 \\
\hline $\mathrm{H}(42 \mathrm{D})$ & 10877 & 8875 & 4301 \\
\hline $\mathrm{H}(42 \mathrm{E})$ & 10279 & 9666 & 4513 \\
\hline $\mathrm{H}(42 \mathrm{~F})$ & 10970 & 9626 & 3897 \\
\hline
\end{tabular}




\begin{tabular}{|c|c|c|c|}
\hline $\mathrm{H}(461)$ & 9040 & 6293 & 3415 \\
\hline $\mathrm{H}(46 \mathrm{~A})$ & 11523 & 6252 & 3003 \\
\hline $\mathrm{H}(46 \mathrm{~B})$ & 10906 & 5555 & 3349 \\
\hline $\mathrm{H}(46 \mathrm{C})$ & 11143 & 6360 & 3677 \\
\hline $\mathrm{H}(46 \mathrm{D})$ & 8363 & 6140 & 2399 \\
\hline $\mathrm{H}(46 \mathrm{E})$ & 9027 & 5389 & 2670 \\
\hline $\mathrm{H}(46 \mathrm{~F})$ & 9767 & 5946 & 2241 \\
\hline $\mathrm{H}(53)$ & 9831 & 9134 & 6798 \\
\hline $\mathrm{H}(54)$ & 9585 & 8186 & 7521 \\
\hline $\mathrm{H}(55)$ & 8516 & 7056 & 7278 \\
\hline $\mathrm{H}(57 \mathrm{~A})$ & 9296 & 8973 & 5309 \\
\hline $\mathrm{H}(57 \mathrm{~B})$ & 9557 & 9603 & 5815 \\
\hline $\mathrm{H}(57 \mathrm{C})$ & 8163 & 9440 & 5576 \\
\hline $\mathrm{H}(58 \mathrm{~A})$ & 7395 & 6264 & 6620 \\
\hline $\mathrm{H}(58 \mathrm{~B})$ & 7806 & 6341 & 5948 \\
\hline $\mathrm{H}(58 \mathrm{C})$ & 6531 & 6721 & 6147 \\
\hline $\mathrm{H}(63)$ & 8311 & 4672 & 3883 \\
\hline $\mathrm{H}(64)$ & 6538 & 4309 & 3337 \\
\hline $\mathrm{H}(65)$ & 4729 & 5040 & 3365 \\
\hline $\mathrm{H}(67 \mathrm{~A})$ & $8500(30)$ & $5896(19)$ & $4990(8)$ \\
\hline $\mathrm{H}(67 \mathrm{~B})$ & $9507(19)$ & $5889(18)$ & $4446(13)$ \\
\hline $\mathrm{H}(68 \mathrm{~A})$ & 4265 & 6668 & 4291 \\
\hline $\mathrm{H}(68 \mathrm{~B})$ & 3536 & 5993 & 3945 \\
\hline $\mathrm{H}(68 \mathrm{C})$ & 4134 & 6682 & 3582 \\
\hline $\mathrm{H}(102)$ & 5008 & 7968 & 4735 \\
\hline $\mathrm{H}(10 \mathrm{~A})$ & 5436 & 7888 & 6080 \\
\hline $\mathrm{H}(10 \mathrm{~B})$ & 4966 & 8607 & 5692 \\
\hline $\mathrm{H}(10 \mathrm{C})$ & 6195 & 8678 & 6109 \\
\hline $\mathrm{H}(10 \mathrm{D})$ & 6748 & 7292 & 3422 \\
\hline $\mathrm{H}(10 \mathrm{E})$ & 6806 & 8198 & 3539 \\
\hline $\mathrm{H}(10 \mathrm{~F})$ & 5500 & 7758 & 3543 \\
\hline $\mathrm{H}(10 \mathrm{G})$ & 6351 & 9270 & 4908 \\
\hline $\mathrm{H}(10 \mathrm{H})$ & 6604 & 9040 & 4239 \\
\hline $\mathrm{H}(10 \mathrm{G})$ & 5878 & 10273 & 3979 \\
\hline $\mathrm{H}(10 \mathrm{H})$ & 5439 & 10490 & 4628 \\
\hline $\mathrm{H}(10 \mathrm{I})$ & 4477 & 10545 & 4072 \\
\hline
\end{tabular}




\begin{tabular}{lllll}
$\mathrm{H}(10 \mathrm{~J})$ & 3086 & 9692 & 4698 & 37 \\
$\mathrm{H}(10 \mathrm{~K})$ & 4196 & 9688 & 5189 & 37 \\
$\mathrm{H}(10 \mathrm{~L})$ & 3632 & 8894 & 4944 & 37 \\
$\mathrm{H}(82)$ & 3017 & 8354 & 4168 & 25 \\
$\mathrm{H}(83)$ & 2241 & 7769 & 3302 & 27 \\
$\mathrm{H}(84)$ & 3000 & 8102 & 2388 & 35 \\
$\mathrm{H}(85)$ & 4526 & 9047 & 2339 & 36 \\
$\mathrm{H}(86)$ & 5289 & 9654 & 3192 & 28 \\
\hline
\end{tabular}






Fully labeled thermal ellipsoid drawing (35\%) of $\mathbf{4 a}$. Hydrogen atoms, disordered atoms, and cocrystallized $\mathrm{CH}_{2} \mathrm{Cl}_{2}$ molecule omitted for clarity. 
Crystal data and structure refinement for $\mathbf{4 a}$.

Identification code

Empirical formula

Formula weight

Temperature

Wavelength

Crystal system

Space group

Unit cell dimensions

Volume

$\mathrm{Z}$

Density (calculated)

Absorption coefficient

$\mathrm{F}(000)$

Crystal size

$\Theta$ range for data collection

Index ranges

Reflections collected

Independent reflections

Completeness to $\Theta=29.57^{\circ}$

Absorption correction

Max. and min. transmission

Refinement method

Data / restraints / parameters

Goodness-of-fit on $\mathrm{F}^{2}$

Final R indices [I>2 $\sigma(\mathrm{I})]$

$\mathrm{R}$ indices (all data)

Largest diff. peak and hole
06192

$\mathrm{C}_{80} \mathrm{H}_{76} \mathrm{BCl}_{2} \mathrm{~F}_{24} \mathrm{MoN}_{3} \mathrm{O}$

$1729.09 \mathrm{~g} / \mathrm{mol}$

100(2) K

$0.71073 \AA$

Triclinic

$\mathrm{P} \overline{1}$

$$
\begin{array}{ll}
\mathrm{a}=11.4290(4) \AA & \alpha=95.7510(10)^{\mathrm{o}} \\
\mathrm{b}=18.7038(7) \AA & \beta=94.0100(10)^{\mathrm{o}} \\
\mathrm{c}=19.0217(7) \AA & \gamma=101.9670(10)^{\mathrm{o}}
\end{array}
$$

3940.6(2) $\AA^{3}$

2

$1.457 \mathrm{~g} / \mathrm{cm}^{3}$

$0.338 \mathrm{~mm}^{-1}$

1764

$0.35 \times 0.25 \times 0.10 \mathrm{~mm}^{3}$

1.65 to $29.57^{\circ}$

$-15 \leq h \leq 15,-25 \leq k \leq 25,-26 \leq l \leq 26$

88758

$22050[\mathrm{R}(\mathrm{int})=0.0316]$

$99.7 \%$

Semi-empirical from equivalents

0.9670 and 0.8909

Full-matrix least-squares on $\mathrm{F}^{2}$

22050 / 2444 / 1192

1.025

$\mathrm{R} 1=0.0407, \mathrm{wR} 2=0.1056$

$\mathrm{R} 1=0.0471, \mathrm{wR} 2=0.1099$

1.103 and $-0.589 \mathrm{e} \cdot \AA^{-3}$ 
Notes on the refinement of $\mathbf{4 a}$.

Five of the eight $\mathrm{CF}_{3}$ groups of the $\mathrm{BAr}_{\mathrm{f} 4}$ anion in the structure of compound $\mathbf{4 a}$ are disordered, and the molecule co-crystallizes with one molecule of $\mathrm{CH}_{2} \mathrm{Cl}_{2}$, which is disordered over two positions. All disorders were refined with the help of similarity restraints on 1-2 and 1-3 distances, and similarity restraints on displacement parameters as well as rigid bond restraints for anisotropic displacement parameters were applied to all atoms of the anion and the solvent molecule. Coordinates for the hydrogen atom on the carbon atom binding directly to the metal (C(1)) were taken from the difference Fourier synthesis and refined semi-freely with the help of a distance restraint, while constraining its $U$-value to 1.2 times the $U_{e q}$ value of C(1). 
Atomic coordinates $\left(\times 10^{4}\right)$ and equivalent isotropic displacement parameters $\left(\AA^{2} \times 10^{3}\right)$ for $\mathbf{4 a}(\mathbf{0 6 1 9 2})$. U(eq) is defined as one third of the trace of the orthogonalized $\mathrm{U}^{\mathrm{ij}}$ tensor.

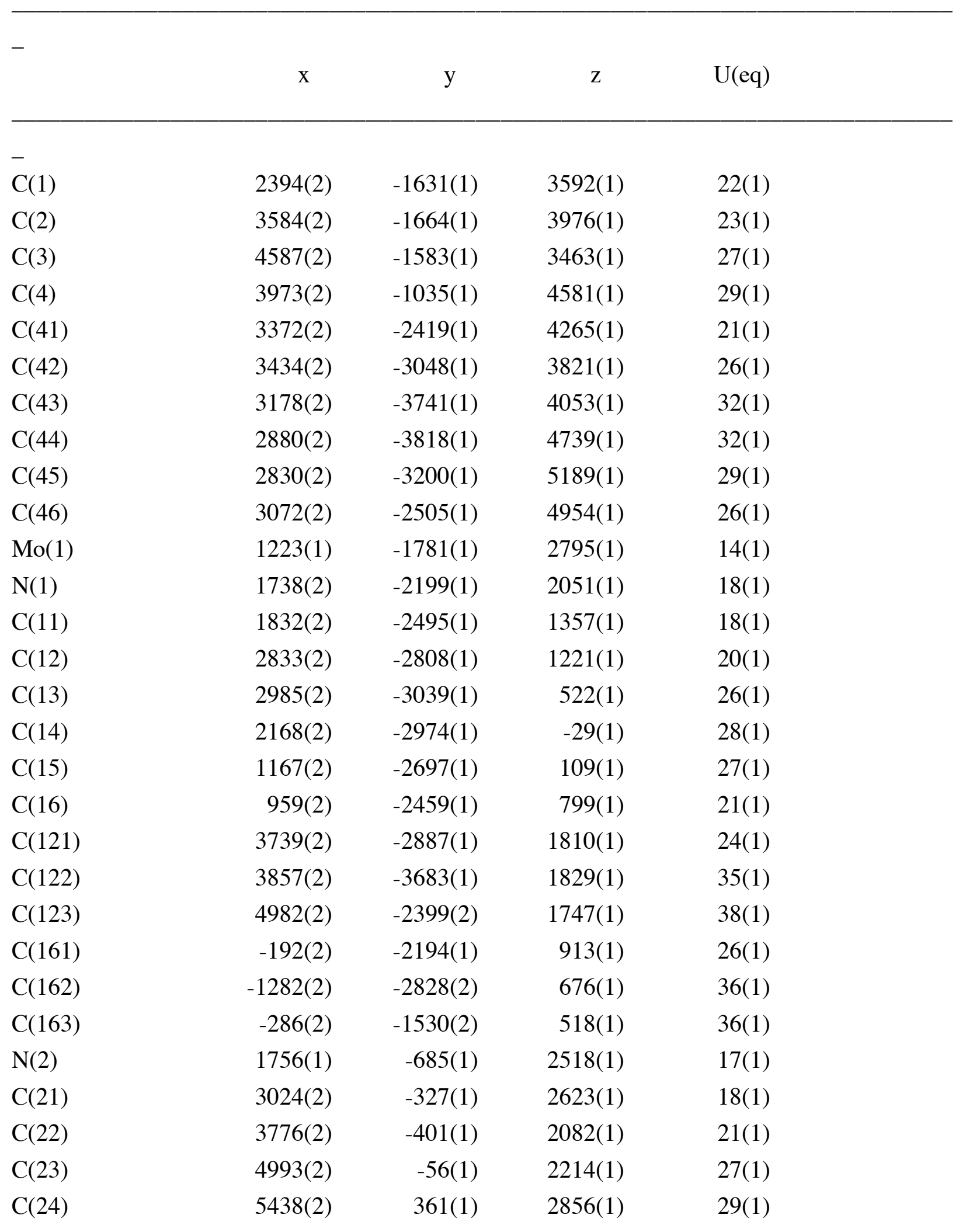




\begin{tabular}{|c|c|c|c|c|}
\hline$C(25)$ & $4665(2)$ & $457(1)$ & $3373(1)$ & $27(1)$ \\
\hline$C(26)$ & $3447(2)$ & $121(1)$ & $3264(1)$ & $21(1)$ \\
\hline$C(27)$ & $3322(2)$ & $-785(1)$ & $1350(1)$ & $27(1)$ \\
\hline $\mathrm{C}(28)$ & $2603(2)$ & 261(1) & $3810(1)$ & $26(1)$ \\
\hline$C(5)$ & $1036(2)$ & $-309(1)$ & $2212(1)$ & $20(1)$ \\
\hline$C(6)$ & $1543(2)$ & $405(1)$ & 1924(1) & $28(1)$ \\
\hline$C(7)$ & $-226(2)$ & $-523(1)$ & $2160(1)$ & $22(1)$ \\
\hline $\mathrm{C}(8)$ & $-921(2)$ & $-1032(1)$ & 2521(1) & $20(1)$ \\
\hline $\mathrm{C}(9)$ & $-2238(2)$ & $-1010(1)$ & $2516(1)$ & $30(1)$ \\
\hline $\mathrm{N}(3)$ & $-481(1)$ & $-1518(1)$ & 2894(1) & $17(1)$ \\
\hline $\mathrm{C}(31)$ & $-1308(2)$ & $-1885(1)$ & $3357(1)$ & $17(1)$ \\
\hline$C(32)$ & $-2104(2)$ & $-2557(1)$ & $3111(1)$ & $21(1)$ \\
\hline$C(33)$ & $-2894(2)$ & $-2882(1)$ & $3573(1)$ & $26(1)$ \\
\hline$C(34)$ & $-2897(2)$ & $-2554(1)$ & $4260(1)$ & $28(1)$ \\
\hline$C(35)$ & $-2125(2)$ & $-1884(1)$ & 4491(1) & $25(1)$ \\
\hline$C(36)$ & $-1329(2)$ & $-1535(1)$ & $4041(1)$ & $20(1)$ \\
\hline$C(37)$ & $-2153(2)$ & $-2917(1)$ & $2363(1)$ & $28(1)$ \\
\hline$C(38)$ & $-554(2)$ & $-781(1)$ & $4285(1)$ & $26(1)$ \\
\hline $\mathrm{O}(1 \mathrm{~T})$ & $358(1)$ & $-2892(1)$ & $3075(1)$ & $18(1)$ \\
\hline $\mathrm{C}(1 \mathrm{~T})$ & $243(2)$ & $-3085(1)$ & 3799(1) & $22(1)$ \\
\hline $\mathrm{C}(2 \mathrm{~T})$ & $-425(2)$ & $-3884(1)$ & $3696(1)$ & $32(1)$ \\
\hline $\mathrm{C}(3 \mathrm{~T})$ & $-49(3)$ & $-4204(1)$ & $3006(1)$ & $39(1)$ \\
\hline $\mathrm{C}(4 \mathrm{~T})$ & $550(2)$ & $-3548(1)$ & $2649(1)$ & $24(1)$ \\
\hline $\mathrm{B}(1)$ & $2639(2)$ & $-3107(1)$ & $-2346(1)$ & $16(1)$ \\
\hline$C(61)$ & $1609(2)$ & $-2970(1)$ & $-2932(1)$ & $17(1)$ \\
\hline$C(62)$ & $1642(2)$ & $-2275(1)$ & $-3161(1)$ & $21(1)$ \\
\hline$C(63)$ & $816(2)$ & $-2163(1)$ & $-3691(1)$ & $26(1)$ \\
\hline$C(67)$ & $859(2)$ & $-1403(2)$ & $-3892(2)$ & $46(1)$ \\
\hline $\mathrm{F}(7)$ & $926(5)$ & $-1483(3)$ & $-4685(2)$ & $61(1)$ \\
\hline $\mathrm{F}(8)$ & $1820(5)$ & $-958(2)$ & $-3720(4)$ & $71(2)$ \\
\hline $\mathrm{F}(9)$ & $-120(5)$ & $-1197(4)$ & $-3893(4)$ & $53(2)$ \\
\hline $\mathrm{F}(7 \mathrm{~A})$ & $1561(8)$ & $-1162(4)$ & $-4333(6)$ & $84(3)$ \\
\hline $\mathrm{F}(8 \mathrm{~A})$ & $1332(7)$ & $-852(2)$ & $-3284(4)$ & $66(2)$ \\
\hline $\mathrm{F}(9 \mathrm{~A})$ & $-158(6)$ & $-1255(5)$ & $-4045(6)$ & $82(3)$ \\
\hline$C(64)$ & $-69(2)$ & $-2741(1)$ & $-4029(1)$ & $24(1)$ \\
\hline$C(65)$ & $-105(2)$ & $-3435(1)$ & $-3823(1)$ & $20(1)$ \\
\hline
\end{tabular}




\begin{tabular}{|c|c|c|c|c|}
\hline$C(68)$ & $-1047(2)$ & $-4081(1)$ & $-4160(1)$ & $27(1)$ \\
\hline $\mathrm{F}(10)$ & $-560(30)$ & $-4577(15)$ & $-4533(18)$ & $37(3)$ \\
\hline $\mathrm{F}(11)$ & $-1680(30)$ & $-4460(20)$ & $-3696(13)$ & $45(4)$ \\
\hline $\mathrm{F}(12)$ & $-1860(30)$ & $-3902(17)$ & $-4614(17)$ & $35(3)$ \\
\hline $\mathrm{F}(10 \mathrm{~A})$ & $-578(18)$ & $-4644(10)$ & $-4411(15)$ & $42(3)$ \\
\hline $\mathrm{F}(11 \mathrm{~A})$ & $-1795(16)$ & $-4342(13)$ & $-3685(8)$ & $42(2)$ \\
\hline $\mathrm{F}(12 \mathrm{~A})$ & $-1720(20)$ & $-3922(12)$ & $-4699(11)$ & $42(3)$ \\
\hline$C(66)$ & $718(2)$ & $-3546(1)$ & $-3288(1)$ & $19(1)$ \\
\hline $\mathrm{C}(71)$ & $2155(2)$ & $-3781(1)$ & $-1879(1)$ & $17(1)$ \\
\hline$C(72)$ & $2973(2)$ & $-4135(1)$ & $-1546(1)$ & $21(1)$ \\
\hline$C(73)$ & $2625(2)$ & $-4663(1)$ & $-1092(1)$ & $24(1)$ \\
\hline$C(77)$ & $3562(2)$ & $-4996(1)$ & $-735(1)$ & $34(1)$ \\
\hline $\mathrm{F}(13)$ & $3127(3)$ & $-5416(2)$ & $-250(2)$ & $76(1)$ \\
\hline $\mathrm{F}(14)$ & $4437(3)$ & $-4495(2)$ & $-409(3)$ & $72(1)$ \\
\hline $\mathrm{F}(15)$ & $3978(5)$ & $-5422(3)$ & $-1187(2)$ & $100(2)$ \\
\hline $\mathrm{F}(13 \mathrm{~A})$ & $3358(9)$ & $-5687(4)$ & $-742(9)$ & $67(4)$ \\
\hline $\mathrm{F}(14 \mathrm{~A})$ & $3853(15)$ & $-4737(9)$ & $-67(5)$ & $86(4)$ \\
\hline $\mathrm{F}(15 \mathrm{~A})$ & $4662(7)$ & $-4860(8)$ & $-991(8)$ & $68(4)$ \\
\hline$C(74)$ & $1431(2)$ & $-4876(1)$ & $-957(1)$ & $26(1)$ \\
\hline$C(75)$ & $609(2)$ & $-4538(1)$ & $-1281(1)$ & $23(1)$ \\
\hline$C(78)$ & $-691(2)$ & $-4738(1)$ & $-1143(1)$ & $32(1)$ \\
\hline $\mathrm{F}(16)$ & $-959(19)$ & $-4265(8)$ & $-633(8)$ & $42(2)$ \\
\hline $\mathrm{F}(17)$ & $-1458(12)$ & $-4735(10)$ & $-1722(7)$ & $33(2)$ \\
\hline $\mathrm{F}(18)$ & $-1017(13)$ & $-5406(6)$ & $-922(10)$ & $43(2)$ \\
\hline $\mathrm{F}(16 \mathrm{~A})$ & $-1060(20)$ & $-4200(11)$ & $-772(17)$ & $49(3)$ \\
\hline $\mathrm{F}(17 \mathrm{~A})$ & $-1373(19)$ & $-4910(20)$ & $-1760(7)$ & $43(3)$ \\
\hline $\mathrm{F}(18 \mathrm{~A})$ & $-900(20)$ & $-5332(12)$ & $-793(17)$ & $58(4)$ \\
\hline$C(76)$ & $959(2)$ & $-3995(1)$ & $-1725(1)$ & $19(1)$ \\
\hline$C(51)$ & $3140(2)$ & $-2385(1)$ & $-1755(1)$ & $18(1)$ \\
\hline$C(52)$ & $4273(2)$ & $-2264(1)$ & $-1375(1)$ & $22(1)$ \\
\hline$C(53)$ & $4662(2)$ & $-1689(1)$ & $-831(1)$ & $27(1)$ \\
\hline$C(57)$ & $5878(2)$ & $-1600(2)$ & $-437(1)$ & $39(1)$ \\
\hline $\mathrm{F}(1)$ & $6290(11)$ & $-933(6)$ & $-103(11)$ & $85(5)$ \\
\hline $\mathrm{F}(2)$ & $6033(14)$ & $-2111(9)$ & $-68(9)$ & $73(4)$ \\
\hline $\mathrm{F}(3)$ & $6743(8)$ & $-1619(8)$ & $-933(5)$ & $57(2)$ \\
\hline $\mathrm{F}(1 \mathrm{~A})$ & $5959(5)$ & $-1199(5)$ & $214(3)$ & $68(2)$ \\
\hline
\end{tabular}




\begin{tabular}{|c|c|c|c|c|}
\hline $\mathrm{F}(2 \mathrm{~A})$ & $6050(9)$ & $-2245(3)$ & $-257(5)$ & $60(2)$ \\
\hline $\mathrm{F}(3 \mathrm{~A})$ & $6763(5)$ & $-1310(5)$ & $-765(4)$ & $64(2)$ \\
\hline $\mathrm{C}(54)$ & $3950(2)$ & $-1194(1)$ & $-653(1)$ & $27(1)$ \\
\hline$C(55)$ & $2824(2)$ & $-1301(1)$ & $-1018(1)$ & $23(1)$ \\
\hline$C(58)$ & $2065(2)$ & $-751(1)$ & $-861(1)$ & $28(1)$ \\
\hline $\mathrm{F}(4)$ & 892(1) & $-1013(1)$ & $-1065(1)$ & $40(1)$ \\
\hline $\mathrm{F}(5)$ & $2136(2)$ & $-507(1)$ & $-172(1)$ & $44(1)$ \\
\hline $\mathrm{F}(6)$ & $2392(2)$ & $-155(1)$ & $-1201(1)$ & $44(1)$ \\
\hline$C(56)$ & $2420(2)$ & $-1888(1)$ & $-1548(1)$ & $21(1)$ \\
\hline $\mathrm{C}(81)$ & $3690(2)$ & $-3302(1)$ & $-2826(1)$ & $17(1)$ \\
\hline $\mathrm{C}(82)$ & $3667(2)$ & $-4025(1)$ & $-3112(1)$ & $19(1)$ \\
\hline$C(83)$ & $4501(2)$ & $-4197(1)$ & $-3561(1)$ & $20(1)$ \\
\hline $\mathrm{C}(87)$ & $4449(2)$ & $-4989(1)$ & $-3811(1)$ & $26(1)$ \\
\hline $\mathrm{F}(19)$ & $3366(2)$ & $-5335(1)$ & $-4116(1)$ & $48(1)$ \\
\hline $\mathrm{F}(20)$ & $5236(2)$ & $-5082(1)$ & $-4278(1)$ & $41(1)$ \\
\hline $\mathrm{F}(21)$ & $4691(2)$ & $-5365(1)$ & $-3272(1)$ & $40(1)$ \\
\hline$C(84)$ & $5403(2)$ & $-3653(1)$ & $-3754(1)$ & $22(1)$ \\
\hline$C(85)$ & $5433(2)$ & $-2931(1)$ & $-3484(1)$ & $20(1)$ \\
\hline $\mathrm{C}(88)$ & $6367(2)$ & $-2321(1)$ & $-3693(1)$ & $26(1)$ \\
\hline $\mathrm{F}(22)$ & $5930(1)$ & $-1981(1)$ & $-4211(1)$ & $33(1)$ \\
\hline $\mathrm{F}(23)$ & $7308(1)$ & $-2553(1)$ & $-3938(1)$ & $39(1)$ \\
\hline $\mathrm{F}(24)$ & $6805(1)$ & $-1797(1)$ & $-3148(1)$ & $36(1)$ \\
\hline$C(86)$ & $4600(2)$ & $-2757(1)$ & $-3030(1)$ & $18(1)$ \\
\hline$C(1 S)$ & $-2390(8)$ & $-3156(8)$ & $-2093(5)$ & $114(4)$ \\
\hline $\mathrm{Cl}(1 \mathrm{~S})$ & $-1151(2)$ & $-2577(1)$ & $-1671(2)$ & $68(1)$ \\
\hline $\mathrm{Cl}(2 \mathrm{~S})$ & $-3408(3)$ & $-3611(2)$ & $-1796(2)$ & $89(1)$ \\
\hline$C(1 R)$ & $-2377(10)$ & $-2776(7)$ & $-1641(6)$ & $107(4)$ \\
\hline $\mathrm{Cl}(1 \mathrm{R})$ & $-1197(2)$ & $-2654(2)$ & $-2180(2)$ & $89(1)$ \\
\hline $\mathrm{Cl}(2 \mathrm{R})$ & $-3343(3)$ & $-3565(2)$ & $-1388(3)$ & $81(1)$ \\
\hline
\end{tabular}


Bond lengths $[\AA]$ and angles $\left[^{\circ}\right]$ for $\mathbf{4 a}(\mathbf{0 6 1 9 2})$.

\begin{tabular}{|c|c|}
\hline- & \\
\hline $\mathrm{C}(1)-\mathrm{C}(2)$ & $1.514(3)$ \\
\hline $\mathrm{C}(1)-\mathrm{Mo}(1)$ & $1.909(2)$ \\
\hline$C(2)-C(4)$ & $1.532(3)$ \\
\hline$C(2)-C(41)$ & $1.546(3)$ \\
\hline$C(2)-C(3)$ & $1.550(3)$ \\
\hline $\mathrm{C}(41)-\mathrm{C}(46)$ & $1.394(3)$ \\
\hline $\mathrm{C}(41)-\mathrm{C}(42)$ & $1.396(3)$ \\
\hline$C(42)-C(43)$ & $1.391(3)$ \\
\hline$C(43)-C(44)$ & $1.386(3)$ \\
\hline$C(44)-C(45)$ & $1.382(3)$ \\
\hline$C(45)-C(46)$ & $1.397(3)$ \\
\hline $\mathrm{Mo}(1)-\mathrm{N}(1)$ & $1.7460(16)$ \\
\hline $\mathrm{Mo}(1)-\mathrm{N}(3)$ & $2.1220(16)$ \\
\hline $\mathrm{Mo}(1)-\mathrm{N}(2)$ & $2.1435(16)$ \\
\hline $\operatorname{Mo}(1)-\mathrm{O}(1 \mathrm{~T})$ & $2.2417(13)$ \\
\hline $\mathrm{N}(1)-\mathrm{C}(11)$ & $1.398(2)$ \\
\hline $\mathrm{C}(11)-\mathrm{C}(12)$ & $1.417(3)$ \\
\hline $\mathrm{C}(11)-\mathrm{C}(16)$ & $1.422(3)$ \\
\hline$C(12)-C(13)$ & $1.390(3)$ \\
\hline$C(12)-C(121)$ & $1.511(3)$ \\
\hline $\mathrm{C}(13)-\mathrm{C}(14)$ & $1.387(3)$ \\
\hline$C(14)-C(15)$ & $1.382(3)$ \\
\hline$C(15)-C(16)$ & $1.394(3)$ \\
\hline$C(16)-C(161)$ & $1.520(3)$ \\
\hline$C(121)-C(122)$ & $1.527(3)$ \\
\hline $\mathrm{C}(121)-\mathrm{C}(123)$ & $1.539(3)$ \\
\hline$C(161)-C(163)$ & $1.533(3)$ \\
\hline$C(161)-C(162)$ & $1.536(3)$ \\
\hline $\mathrm{N}(2)-\mathrm{C}(5)$ & $1.329(2)$ \\
\hline $\mathrm{N}(2)-\mathrm{C}(21)$ & $1.456(2)$ \\
\hline $\mathrm{C}(21)-\mathrm{C}(22)$ & $1.399(3)$ \\
\hline$C(21)-C(26)$ & $1.403(3)$ \\
\hline $\mathrm{C}(22)-\mathrm{C}(23)$ & $1.400(3)$ \\
\hline
\end{tabular}




\begin{tabular}{|c|c|}
\hline $\mathrm{C}(22)-\mathrm{C}(27)$ & $1.507(3)$ \\
\hline $\mathrm{C}(23)-\mathrm{C}(24)$ & $1.384(3)$ \\
\hline$C(24)-C(25)$ & $1.390(3)$ \\
\hline $\mathrm{C}(25)-\mathrm{C}(26)$ & $1.395(3)$ \\
\hline $\mathrm{C}(26)-\mathrm{C}(28)$ & $1.506(3)$ \\
\hline$C(5)-C(7)$ & $1.408(3)$ \\
\hline$C(5)-C(6)$ & $1.513(3)$ \\
\hline$C(7)-C(8)$ & $1.381(3)$ \\
\hline $\mathrm{C}(8)-\mathrm{N}(3)$ & $1.360(2)$ \\
\hline $\mathrm{C}(8)-\mathrm{C}(9)$ & $1.514(3)$ \\
\hline $\mathrm{N}(3)-\mathrm{C}(31)$ & $1.452(2)$ \\
\hline $\mathrm{C}(31)-\mathrm{C}(36)$ & $1.402(3)$ \\
\hline $\mathrm{C}(31)-\mathrm{C}(32)$ & $1.405(3)$ \\
\hline C(32)-C(33) & $1.396(3)$ \\
\hline$C(32)-C(37)$ & $1.503(3)$ \\
\hline $\mathrm{C}(33)-\mathrm{C}(34)$ & $1.388(3)$ \\
\hline$C(34)-C(35)$ & $1.385(3)$ \\
\hline $\mathrm{C}(35)-\mathrm{C}(36)$ & $1.400(3)$ \\
\hline$C(36)-C(38)$ & $1.510(3)$ \\
\hline $\mathrm{O}(1 \mathrm{~T})-\mathrm{C}(4 \mathrm{~T})$ & $1.464(2)$ \\
\hline $\mathrm{O}(1 \mathrm{~T})-\mathrm{C}(1 \mathrm{~T})$ & $1.467(2)$ \\
\hline $\mathrm{C}(1 \mathrm{~T})-\mathrm{C}(2 \mathrm{~T})$ & $1.517(3)$ \\
\hline $\mathrm{C}(2 \mathrm{~T})-\mathrm{C}(3 \mathrm{~T})$ & $1.515(3)$ \\
\hline $\mathrm{C}(3 \mathrm{~T})-\mathrm{C}(4 \mathrm{~T})$ & $1.522(3)$ \\
\hline $\mathrm{B}(1)-\mathrm{C}(81)$ & $1.637(3)$ \\
\hline $\mathrm{B}(1)-\mathrm{C}(71)$ & $1.640(3)$ \\
\hline $\mathrm{B}(1)-\mathrm{C}(61)$ & $1.640(3)$ \\
\hline $\mathrm{B}(1)-\mathrm{C}(51)$ & $1.643(3)$ \\
\hline $\mathrm{C}(61)-\mathrm{C}(66)$ & $1.399(3)$ \\
\hline $\mathrm{C}(61)-\mathrm{C}(62)$ & $1.406(3)$ \\
\hline $\mathrm{C}(62)-\mathrm{C}(63)$ & $1.393(3)$ \\
\hline $\mathrm{C}(63)-\mathrm{C}(64)$ & $1.387(3)$ \\
\hline $\mathrm{C}(63)-\mathrm{C}(67)$ & $1.500(3)$ \\
\hline $\mathrm{C}(67)-\mathrm{F}(8)$ & $1.234(4)$ \\
\hline $\mathrm{C}(67)-\mathrm{F}(7 \mathrm{~A})$ & $1.254(5)$ \\
\hline $\mathrm{C}(67)-\mathrm{F}(9)$ & $1.257(6)$ \\
\hline
\end{tabular}




\begin{tabular}{|c|c|}
\hline $\mathrm{C}(67)-\mathrm{F}(9 \mathrm{~A})$ & $1.270(7)$ \\
\hline $\mathrm{C}(67)-\mathrm{F}(8 \mathrm{~A})$ & $1.460(6)$ \\
\hline $\mathrm{C}(67)-\mathrm{F}(7)$ & $1.510(5)$ \\
\hline$C(64)-C(65)$ & $1.386(3)$ \\
\hline$C(65)-C(66)$ & $1.398(3)$ \\
\hline$C(65)-C(68)$ & $1.496(3)$ \\
\hline $\mathrm{C}(68)-\mathrm{F}(12 \mathrm{~A})$ & $1.333(9)$ \\
\hline $\mathrm{C}(68)-\mathrm{F}(12)$ & $1.336(13)$ \\
\hline $\mathrm{C}(68)-\mathrm{F}(10 \mathrm{~A})$ & $1.340(9)$ \\
\hline $\mathrm{C}(68)-\mathrm{F}(11)$ & $1.341(13)$ \\
\hline $\mathrm{C}(68)-\mathrm{F}(10)$ & $1.343(13)$ \\
\hline $\mathrm{C}(68)-\mathrm{F}(11 \mathrm{~A})$ & $1.345(8)$ \\
\hline $\mathrm{C}(71)-\mathrm{C}(76)$ & $1.402(3)$ \\
\hline $\mathrm{C}(71)-\mathrm{C}(72)$ & $1.405(3)$ \\
\hline$C(72)-C(73)$ & $1.390(3)$ \\
\hline$C(73)-C(74)$ & $1.390(3)$ \\
\hline$C(73)-C(77)$ & $1.501(3)$ \\
\hline $\mathrm{C}(77)-\mathrm{F}(13 \mathrm{~A})$ & $1.265(7)$ \\
\hline $\mathrm{C}(77)-\mathrm{F}(14)$ & $1.294(4)$ \\
\hline $\mathrm{C}(77)-\mathrm{F}(15)$ & $1.295(4)$ \\
\hline $\mathrm{C}(77)-\mathrm{F}(14 \mathrm{~A})$ & $1.309(8)$ \\
\hline $\mathrm{C}(77)-\mathrm{F}(13)$ & $1.329(3)$ \\
\hline $\mathrm{C}(77)-\mathrm{F}(15 \mathrm{~A})$ & $1.364(8)$ \\
\hline $\mathrm{C}(74)-\mathrm{C}(75)$ & $1.380(3)$ \\
\hline$C(75)-C(76)$ & $1.398(3)$ \\
\hline $\mathrm{C}(75)-\mathrm{C}(78)$ & $1.503(3)$ \\
\hline $\mathrm{C}(78)-\mathrm{F}(16 \mathrm{~A})$ & $1.326(11)$ \\
\hline $\mathrm{C}(78)-\mathrm{F}(17 \mathrm{~A})$ & $1.333(11)$ \\
\hline $\mathrm{C}(78)-\mathrm{F}(18 \mathrm{~A})$ & $1.340(11)$ \\
\hline $\mathrm{C}(78)-\mathrm{F}(16)$ & $1.341(9)$ \\
\hline $\mathrm{C}(78)-\mathrm{F}(18)$ & $1.347(8)$ \\
\hline $\mathrm{C}(78)-\mathrm{F}(17)$ & $1.360(8)$ \\
\hline $\mathrm{C}(51)-\mathrm{C}(52)$ & $1.403(3)$ \\
\hline$C(51)-C(56)$ & $1.408(3)$ \\
\hline$C(52)-C(53)$ & $1.393(3)$ \\
\hline$C(53)-C(54)$ & $1.387(3)$ \\
\hline
\end{tabular}




\begin{tabular}{|c|c|}
\hline$C(53)-C(57)$ & $1.500(3)$ \\
\hline $\mathrm{C}(57)-\mathrm{F}(2)$ & $1.275(11)$ \\
\hline $\mathrm{C}(57)-\mathrm{F}(3 \mathrm{~A})$ & $1.278(6)$ \\
\hline $\mathrm{C}(57)-\mathrm{F}(1)$ & $1.319(7)$ \\
\hline $\mathrm{C}(57)-\mathrm{F}(2 \mathrm{~A})$ & $1.335(6)$ \\
\hline $\mathrm{C}(57)-\mathrm{F}(1 \mathrm{~A})$ & $1.369(4)$ \\
\hline $\mathrm{C}(57)-\mathrm{F}(3)$ & $1.416(9)$ \\
\hline$C(54)-C(55)$ & $1.386(3)$ \\
\hline$C(55)-C(56)$ & $1.392(3)$ \\
\hline $\mathrm{C}(55)-\mathrm{C}(58)$ & $1.499(3)$ \\
\hline $\mathrm{C}(58)-\mathrm{F}(5)$ & $1.335(2)$ \\
\hline $\mathrm{C}(58)-\mathrm{F}(6)$ & $1.343(3)$ \\
\hline $\mathrm{C}(58)-\mathrm{F}(4)$ & $1.344(3)$ \\
\hline $\mathrm{C}(81)-\mathrm{C}(82)$ & $1.401(3)$ \\
\hline $\mathrm{C}(81)-\mathrm{C}(86)$ & $1.402(3)$ \\
\hline $\mathrm{C}(82)-\mathrm{C}(83)$ & $1.388(3)$ \\
\hline$C(83)-C(84)$ & $1.388(3)$ \\
\hline $\mathrm{C}(83)-\mathrm{C}(87)$ & $1.498(3)$ \\
\hline $\mathrm{C}(87)-\mathrm{F}(20)$ & $1.331(3)$ \\
\hline $\mathrm{C}(87)-\mathrm{F}(19)$ & $1.333(3)$ \\
\hline $\mathrm{C}(87)-\mathrm{F}(21)$ & $1.345(3)$ \\
\hline$C(84)-C(85)$ & $1.388(3)$ \\
\hline$C(85)-C(86)$ & $1.395(3)$ \\
\hline $\mathrm{C}(85)-\mathrm{C}(88)$ & $1.498(3)$ \\
\hline $\mathrm{C}(88)-\mathrm{F}(23)$ & $1.336(2)$ \\
\hline $\mathrm{C}(88)-\mathrm{F}(24)$ & $1.344(3)$ \\
\hline $\mathrm{C}(88)-\mathrm{F}(22)$ & $1.347(3)$ \\
\hline $\mathrm{C}(1 \mathrm{~S})-\mathrm{Cl}(2 \mathrm{~S})$ & $1.475(9)$ \\
\hline $\mathrm{C}(1 \mathrm{~S})-\mathrm{Cl}(1 \mathrm{~S})$ & $1.690(8)$ \\
\hline $\mathrm{C}(1 \mathrm{R})-\mathrm{Cl}(1 \mathrm{R})$ & $1.741(8)$ \\
\hline $\mathrm{C}(1 \mathrm{R})-\mathrm{Cl}(2 \mathrm{R})$ & $1.782(11)$ \\
\hline $\mathrm{C}(2)-\mathrm{C}(1)-\mathrm{Mo}(1)$ & $155.26(17)$ \\
\hline$C(1)-C(2)-C(4)$ & 111.12(18) \\
\hline$C(1)-C(2)-C(41)$ & 105.34(16) \\
\hline$C(4)-C(2)-C(41)$ & $110.95(17)$ \\
\hline
\end{tabular}




\begin{tabular}{|c|c|}
\hline$C(1)-C(2)-C(3)$ & $110.59(17)$ \\
\hline $\mathrm{C}(4)-\mathrm{C}(2)-\mathrm{C}(3)$ & $107.04(18)$ \\
\hline$C(41)-C(2)-C(3)$ & $111.86(17)$ \\
\hline$C(46)-C(41)-C(42)$ & $7.85(19)$ \\
\hline $\mathrm{C}(46)-\mathrm{C}(41)-\mathrm{C}(2)$ & $122.31(19)$ \\
\hline $\mathrm{C}(42)-\mathrm{C}(41)-\mathrm{C}(2)$ & $119.80(18)$ \\
\hline$C(43)-C(42)-C(41)$ & $121.1(2)$ \\
\hline $\mathrm{C}(44)-\mathrm{C}(43)-\mathrm{C}(42)$ & $120.3(2)$ \\
\hline$C(45)-C(44)-C(43)$ & $119.3(2)$ \\
\hline$C(44)-C(45)-C(46)$ & $120.3(2)$ \\
\hline$C(41)-C(46)-C(45)$ & $121.0(2)$ \\
\hline $\mathrm{N}(1)-\mathrm{Mo}(1)-\mathrm{C}(1)$ & $110.49(9)$ \\
\hline $\mathrm{N}(1)-\mathrm{Mo}(1)-\mathrm{N}(3)$ & $128.22(7)$ \\
\hline $\mathrm{C}(1)-\mathrm{Mo}(1)-\mathrm{N}(3)$ & $121.16(8)$ \\
\hline $\mathrm{N}(1)-\mathrm{Mo}(1)-\mathrm{N}(2)$ & $94.91(7)$ \\
\hline $\mathrm{C}(1)-\mathrm{Mo}(1)-\mathrm{N}(2)$ & $94.55(7)$ \\
\hline $\mathrm{N}(3)-\mathrm{Mo}(1)-\mathrm{N}(2)$ & $84.99(6)$ \\
\hline $\mathrm{N}(1)-\mathrm{Mo}(1)-\mathrm{O}(1 \mathrm{~T})$ & $6(6)$ \\
\hline $\mathrm{C}(1)-\mathrm{Mo}(1)-\mathrm{O}(1 \mathrm{~T})$ & $91.43(7)$ \\
\hline $\mathrm{N}(3)-\mathrm{Mo}(1)-\mathrm{O}(1 \mathrm{~T})$ & $85.63(5)$ \\
\hline $\mathrm{N}(2)-\mathrm{Mo}(1)-\mathrm{O}(1 \mathrm{~T})$ & $170.53(5)$ \\
\hline $\mathrm{C}(11)-\mathrm{N}(1)-\mathrm{Mo}(1)$ & $162.68(15)$ \\
\hline$N(1)-C(11)-C(12)$ & $118.58(17)$ \\
\hline $\mathrm{N}(1)-\mathrm{C}(11)-\mathrm{C}(16)$ & $120.44(18)$ \\
\hline$C(12)-C(11)-C(16)$ & $120.95(17)$ \\
\hline$C(13)-C(12)-C(11)$ & $118.56(19)$ \\
\hline$C(13)-C(12)-C(121)$ & $119.48(19)$ \\
\hline$C(11)-C(12)-C(121)$ & $121.95(17)$ \\
\hline $\mathrm{C}(14)-\mathrm{C}(13)-\mathrm{C}(12)$ & $120.8(2)$ \\
\hline$C(15)-C(14)-C(13)$ & $120.36(19)$ \\
\hline$C(14)-C(15)-C(16)$ & $121.6(2)$ \\
\hline$C(15)-C(16)-C(11)$ & $117.55(19)$ \\
\hline$C(15)-C(16)-C(161)$ & $118.54(18)$ \\
\hline$C(11)-C(16)-C(161)$ & $123.89(17)$ \\
\hline $\mathrm{C}(12)-\mathrm{C}(121)-\mathrm{C}(122)$ & $112.36(18)$ \\
\hline$C(12)-C(121)-C(123)$ & $111.31(18)$ \\
\hline
\end{tabular}




\begin{tabular}{|c|c|}
\hline$C(122)-C(121)-C(123)$ & $109.4(2)$ \\
\hline$C(16)-C(161)-C(163)$ & $112.39(18)$ \\
\hline$C(16)-C(161)-C(162)$ & $109.62(19)$ \\
\hline$C(163)-C(161)-C(162)$ & $110.1(2)$ \\
\hline $\mathrm{C}(5)-\mathrm{N}(2)-\mathrm{C}(21)$ & $115.71(16)$ \\
\hline $\mathrm{C}(5)-\mathrm{N}(2)-\mathrm{Mo}(1)$ & $125.61(13)$ \\
\hline $\mathrm{C}(21)-\mathrm{N}(2)-\mathrm{Mo}(1)$ & $118.53(12)$ \\
\hline$C(22)-C(21)-C(26)$ & $21.47(18)$ \\
\hline $\mathrm{C}(22)-\mathrm{C}(21)-\mathrm{N}(2)$ & $120.53(17)$ \\
\hline $\mathrm{C}(26)-\mathrm{C}(21)-\mathrm{N}(2)$ & $117.92(17)$ \\
\hline$C(21)-C(22)-C(23)$ & $118.32(19)$ \\
\hline$C(21)-C(22)-C(27)$ & $123.08(18)$ \\
\hline$C(23)-C(22)-C(27)$ & $118.44(19)$ \\
\hline$C(24)-C(23)-C(22)$ & $121.0(2)$ \\
\hline$C(23)-C(24)-C(25)$ & $119.8(2)$ \\
\hline$C(24)-C(25)-C(26)$ & $121.0(2)$ \\
\hline$C(25)-C(26)-C(21)$ & $118.27(19)$ \\
\hline$C(25)-C(26)-C(28)$ & $120.67(19)$ \\
\hline$C(21)-C(26)-C(28)$ & 121.01(18) \\
\hline$N(2)-C(5)-C(7)$ & $123.25(17)$ \\
\hline$N(2)-C(5)-C(6)$ & $120.88(17)$ \\
\hline$C(7)-C(5)-C(6)$ & $115.81(17)$ \\
\hline $\mathrm{C}(8)-\mathrm{C}(7)-\mathrm{C}(5)$ & $127.68(18)$ \\
\hline $\mathrm{N}(3)-\mathrm{C}(8)-\mathrm{C}(7)$ & $124.02(18)$ \\
\hline $\mathrm{N}(3)-\mathrm{C}(8)-\mathrm{C}(9)$ & $119.63(17)$ \\
\hline $\mathrm{C}(7)-\mathrm{C}(8)-\mathrm{C}(9)$ & $116.29(17)$ \\
\hline $\mathrm{C}(8)-\mathrm{N}(3)-\mathrm{C}(31)$ & $114.28(15)$ \\
\hline $\mathrm{C}(8)-\mathrm{N}(3)-\mathrm{Mo}(1)$ & $124.57(13)$ \\
\hline $\mathrm{C}(31)-\mathrm{N}(3)-\mathrm{Mo}(1)$ & $121.09(12)$ \\
\hline$C(36)-C(31)-C(32)$ & $121.09(17)$ \\
\hline $\mathrm{C}(36)-\mathrm{C}(31)-\mathrm{N}(3)$ & $118.23(16)$ \\
\hline $\mathrm{C}(32)-\mathrm{C}(31)-\mathrm{N}(3)$ & $120.61(17)$ \\
\hline $\mathrm{C}(33)-\mathrm{C}(32)-\mathrm{C}(31)$ & $118.38(19)$ \\
\hline$C(33)-C(32)-C(37)$ & $119.62(18)$ \\
\hline $\mathrm{C}(31)-\mathrm{C}(32)-\mathrm{C}(37)$ & $121.97(18)$ \\
\hline$C(34)-C(33)-C(32)$ & $121.16(19)$ \\
\hline
\end{tabular}




\begin{tabular}{|c|c|}
\hline$C(35)-C(34)-C(33)$ & $119.82(19)$ \\
\hline$C(34)-C(35)-C(36)$ & $120.8(2)$ \\
\hline$C(35)-C(36)-C(31)$ & $118.64(18)$ \\
\hline$C(35)-C(36)-C(38)$ & $120.03(18)$ \\
\hline$C(31)-C(36)-C(38)$ & $121.27(17)$ \\
\hline $\mathrm{C}(4 \mathrm{~T})-\mathrm{O}(1 \mathrm{~T})-\mathrm{C}(1 \mathrm{~T})$ & $106.48(14)$ \\
\hline $\mathrm{C}(4 \mathrm{~T})-\mathrm{O}(1 \mathrm{~T})-\mathrm{Mo}(1)$ & $118.66(11)$ \\
\hline $\mathrm{C}(1 \mathrm{~T})-\mathrm{O}(1 \mathrm{~T})-\mathrm{Mo}(1)$ & $125.08(11)$ \\
\hline $\mathrm{O}(1 \mathrm{~T})-\mathrm{C}(1 \mathrm{~T})-\mathrm{C}(2 \mathrm{~T})$ & $104.08(16)$ \\
\hline $\mathrm{C}(3 \mathrm{~T})-\mathrm{C}(2 \mathrm{~T})-\mathrm{C}(1 \mathrm{~T})$ & $104.85(18)$ \\
\hline $\mathrm{C}(2 \mathrm{~T})-\mathrm{C}(3 \mathrm{~T})-\mathrm{C}(4 \mathrm{~T})$ & $105.88(19)$ \\
\hline $\mathrm{O}(1 \mathrm{~T})-\mathrm{C}(4 \mathrm{~T})-\mathrm{C}(3 \mathrm{~T})$ & $106.16(17)$ \\
\hline $\mathrm{C}(81)-\mathrm{B}(1)-\mathrm{C}(71)$ & $110.46(15)$ \\
\hline $\mathrm{C}(81)-\mathrm{B}(1)-\mathrm{C}(61)$ & $103.78(14)$ \\
\hline $\mathrm{C}(71)-\mathrm{B}(1)-\mathrm{C}(61)$ & $113.52(15)$ \\
\hline $\mathrm{C}(81)-\mathrm{B}(1)-\mathrm{C}(51)$ & $112.24(15)$ \\
\hline $\mathrm{C}(71)-\mathrm{B}(1)-\mathrm{C}(51)$ & $104.77(14)$ \\
\hline $\mathrm{C}(61)-\mathrm{B}(1)-\mathrm{C}(51)$ & $112.29(15)$ \\
\hline$C(66)-C(61)-C(62)$ & $115.54(17)$ \\
\hline $\mathrm{C}(66)-\mathrm{C}(61)-\mathrm{B}(1)$ & $122.41(16)$ \\
\hline $\mathrm{C}(62)-\mathrm{C}(61)-\mathrm{B}(1)$ & $121.75(17)$ \\
\hline$C(63)-C(62)-C(61)$ & $122.20(19)$ \\
\hline$C(64)-C(63)-C(62)$ & $121.14(19)$ \\
\hline$C(64)-C(63)-C(67)$ & $118.9(2)$ \\
\hline$C(62)-C(63)-C(67)$ & $119.9(2)$ \\
\hline $\mathrm{F}(8)-\mathrm{C}(67)-\mathrm{F}(7 \mathrm{~A})$ & $57.0(5)$ \\
\hline $\mathrm{F}(8)-\mathrm{C}(67)-\mathrm{F}(9)$ & $120.6(5)$ \\
\hline $\mathrm{F}(7 \mathrm{~A})-\mathrm{C}(67)-\mathrm{F}(9)$ & $117.0(5)$ \\
\hline $\mathrm{F}(8)-\mathrm{C}(67)-\mathrm{F}(9 \mathrm{~A})$ & $126.8(5)$ \\
\hline $\mathrm{F}(7 \mathrm{~A})-\mathrm{C}(67)-\mathrm{F}(9 \mathrm{~A})$ & $108.9(6)$ \\
\hline $\mathrm{F}(9)-\mathrm{C}(67)-\mathrm{F}(9 \mathrm{~A})$ & $13.4(7)$ \\
\hline $\mathrm{F}(8)-\mathrm{C}(67)-\mathrm{F}(8 \mathrm{~A})$ & $45.2(3)$ \\
\hline $\mathrm{F}(7 \mathrm{~A})-\mathrm{C}(67)-\mathrm{F}(8 \mathrm{~A})$ & $99.2(5)$ \\
\hline $\mathrm{F}(9)-\mathrm{C}(67)-\mathrm{F}(8 \mathrm{~A})$ & $89.0(5)$ \\
\hline $\mathrm{F}(9 \mathrm{~A})-\mathrm{C}(67)-\mathrm{F}(8 \mathrm{~A})$ & $101.2(6)$ \\
\hline$F(8)-C(67)-C(63)$ & $115.4(3)$ \\
\hline
\end{tabular}




$\begin{array}{lc}F(7 A)-C(67)-C(63) & 119.3(3) \\ F(9)-C(67)-C(63) & 115.2(4) \\ F(9 A)-C(67)-C(63) & 115.1(5) \\ F(8 A)-C(67)-C(63) & 110.4(3) \\ F(8)-C(67)-F(7) & 98.3(4) \\ F(7 A)-C(67)-F(7) & 41.3(5) \\ F(9)-C(67)-F(7) & 97.3(4) \\ F(9 A)-C(67)-F(7) & 84.4(6) \\ F(8 A)-C(67)-F(7) & 137.8(4) \\ C(63)-C(67)-F(7) & 104.4(3) \\ C(65)-C(64)-C(63) & 117.80(18) \\ C(64)-C(65)-C(66) & 121.04(18) \\ C(64)-C(65)-C(68) & 120.52(18) \\ C(66)-C(65)-C(68) & 118.43(18) \\ F(12 A)-C(68)-F(12) & 10(2) \\ F(12 A)-C(68)-F(10 A) & 106.9(10) \\ F(12)-C(68)-F(10 A) & 114.6(19) \\ F(12 A)-C(68)-F(11) & 114.1(19) \\ F(12)-C(68)-F(11) & 106.1(16) \\ F(10 A)-C(68)-F(11) & 94.4(18) \\ F(12 A)-C(68)-F(10) & 97.4(19) \\ F(12)-C(68)-F(10) & 105.9(16) \\ F(10 A)-C(68)-F(10) & 12(2) \\ F(11)-C(68)-F(10) & 104.9(16) \\ F(12 A)-C(68)-F(11 A) & 107.0(10) \\ F(12)-C(68)-F(11 A) & 98(2) \\ F(10 A)-C(68)-F(11 A) & 106.0(9) \\ F(11)-C(68)-F(11 A) & 12(2) \\ F(10)-C(68)-F(11 A) & 116.1(18) \\ F(12 A)-C(68)-C(65) & 113.2(9) \\ F(12)-C(68)-C(65) & 113.5(14) \\ F(10 A)-C(68)-C(65) & 112.5(9) \\ F(11)-C(68)-C(65) & 114.1(13) \\ F(10)-C(68)-C(65) & 111.6(15) \\ C(65)-C(66)-C(61) & 122.25(18) \\ & \end{array}$




\begin{tabular}{|c|c|}
\hline$C(76)-C(71)-C(72)$ & $115.58(17)$ \\
\hline $\mathrm{C}(76)-\mathrm{C}(71)-\mathrm{B}(1)$ & $124.07(17)$ \\
\hline $\mathrm{C}(72)-\mathrm{C}(71)-\mathrm{B}(1)$ & $120.07(16)$ \\
\hline $\mathrm{C}(73)-\mathrm{C}(72)-\mathrm{C}(71)$ & $122.40(19)$ \\
\hline$C(72)-C(73)-C(74)$ & $121.01(19)$ \\
\hline$C(72)-C(73)-C(77)$ & 119.2(2) \\
\hline $\mathrm{C}(74)-\mathrm{C}(73)-\mathrm{C}(77)$ & 119.82(19) \\
\hline $\mathrm{F}(13 \mathrm{~A})-\mathrm{C}(77)-\mathrm{F}(14)$ & $129.7(5)$ \\
\hline $\mathrm{F}(13 \mathrm{~A})-\mathrm{C}(77)-\mathrm{F}(15)$ & $56.9(7)$ \\
\hline $\mathrm{F}(14)-\mathrm{C}(77)-\mathrm{F}(15)$ & $109.6(4)$ \\
\hline $\mathrm{F}(13 \mathrm{~A})-\mathrm{C}(77)-\mathrm{F}(14 \mathrm{~A})$ & 104.7(9) \\
\hline $\mathrm{F}(14)-\mathrm{C}(77)-\mathrm{F}(14 \mathrm{~A})$ & \\
\hline $\mathrm{F}(15)-\mathrm{C}(77)-\mathrm{F}(14 \mathrm{~A})$ & $134.6(5)$ \\
\hline $\mathrm{F}(13 \mathrm{~A})-\mathrm{C}(77)-\mathrm{F}($ & \\
\hline $\mathrm{F}(14)-\mathrm{C}(77)-\mathrm{F}(13)$ & $106.2(3)$ \\
\hline $\mathrm{F}(15)-\mathrm{C}(77)-\mathrm{F}(13)$ & $105.6(3)$ \\
\hline $\mathrm{F}(14 \mathrm{~A})-\mathrm{C}(77)-\mathrm{F}(13)$ & $62.2(9)$ \\
\hline $\mathrm{F}(13 \mathrm{~A})-\mathrm{C}(77)-\mathrm{F}(15 \mathrm{~A})$ & 101. \\
\hline $\mathrm{F}(14)-\mathrm{C}(77)-\mathrm{F}(15 \mathrm{~A})$ & $59.1(7)$ \\
\hline $\mathrm{F}(15)-\mathrm{C}(77)-\mathrm{F}(15 \mathrm{~A})$ & $52.6(6)$ \\
\hline $\mathrm{F}(14 \mathrm{~A})-\mathrm{C}(77)-\mathrm{F}(15 \mathrm{~A})$ & $100.6(9)$ \\
\hline $\mathrm{F}(13)-\mathrm{C}(77)-\mathrm{F}(15 \mathrm{~A})$ & $131.5(4)$ \\
\hline $\mathrm{F}(13 \mathrm{~A})-\mathrm{C}(77)-\mathrm{C}(73)$ & $118.4(5)$ \\
\hline$F(14)-C(77)-C(73)$ & $111.5(2)$ \\
\hline$F(15)-C(77)-C(73)$ & $111.2(2)$ \\
\hline $\mathrm{F}(14 \mathrm{~A})-\mathrm{C}(77)-\mathrm{C}(73)$ & $113.7(4)$ \\
\hline $\mathrm{F}(13)-\mathrm{C}(77)-\mathrm{C}(73)$ & $112.4(2)$ \\
\hline $\mathrm{F}(15 \mathrm{~A})-\mathrm{C}(77)-\mathrm{C}(73)$ & $115.9(4)$ \\
\hline$C(75)-C(74)-C(73)$ & $117.67(18)$ \\
\hline$C(74)-C(75)-C(76)$ & $121.52(19)$ \\
\hline $\mathrm{C}(74)-\mathrm{C}(75)-\mathrm{C}(78)$ & 119.91(19) \\
\hline $\mathrm{C}(76)-\mathrm{C}(75)-\mathrm{C}(78)$ & $118.5(2)$ \\
\hline $\mathrm{F}(16 \mathrm{~A})-\mathrm{C}(78)-\mathrm{F}(17 \mathrm{~A})$ & $108.3(10)$ \\
\hline $\mathrm{F}(16 \mathrm{~A})-\mathrm{C}(78)-\mathrm{F}(18 \mathrm{~A})$ & $108.2(12)$ \\
\hline $\mathrm{F}(17 \mathrm{~A})-\mathrm{C}(78)-\mathrm{F}(18 \mathrm{~A})$ & $106.9(10)$ \\
\hline $\mathrm{F}(16 \mathrm{~A})-\mathrm{C}(78)-\mathrm{F}(16)$ & $13.9(15)$ \\
\hline
\end{tabular}




\begin{tabular}{|c|c|}
\hline $\mathrm{F}(17 \mathrm{~A})-\mathrm{C}(78)-\mathrm{F}(16)$ & $120.4(14)$ \\
\hline $\mathrm{F}(18 \mathrm{~A})-\mathrm{C}(78)-\mathrm{F}(16)$ & $96.8(13)$ \\
\hline $\mathrm{F}(16 \mathrm{~A})-\mathrm{C}(78)-\mathrm{F}(18)$ & $115.1(14)$ \\
\hline $\mathrm{F}(17 \mathrm{~A})-\mathrm{C}(78)-\mathrm{F}(18)$ & $95.2(13)$ \\
\hline $\mathrm{F}(18 \mathrm{~A})-\mathrm{C}(78)-\mathrm{F}(18)$ & $12.0(17)$ \\
\hline $\mathrm{F}(16)-\mathrm{C}(78)-\mathrm{F}(18)$ & $105.3(9)$ \\
\hline $\mathrm{F}(16 \mathrm{~A})-\mathrm{C}(78)-\mathrm{F}(17)$ & $93.2(12)$ \\
\hline $\mathrm{F}(17 \mathrm{~A})-\mathrm{C}(78)-\mathrm{F}(17)$ & $15.7(13)$ \\
\hline $\mathrm{F}(18 \mathrm{~A})-\mathrm{C}(78)-\mathrm{F}(17)$ & $117.3(13)$ \\
\hline $\mathrm{F}(16)-\mathrm{C}(78)-\mathrm{F}(17)$ & $106.0(8)$ \\
\hline $\mathrm{F}(18)-\mathrm{C}(78)-\mathrm{F}(17)$ & $106.3(8)$ \\
\hline $\mathrm{F}(16 \mathrm{~A})-\mathrm{C}(78)-\mathrm{C}(75)$ & $113.3(11)$ \\
\hline $\mathrm{F}(17 \mathrm{~A})-\mathrm{C}(78)-\mathrm{C}(75)$ & $109.5(10)$ \\
\hline $\mathrm{F}(18 \mathrm{~A})-\mathrm{C}(78)-\mathrm{C}(75)$ & $110.5(9)$ \\
\hline $\mathrm{F}(16)-\mathrm{C}(78)-\mathrm{C}(75)$ & 111.7(9) \\
\hline $\mathrm{F}(18)-\mathrm{C}(78)-\mathrm{C}(75)$ & $113.7(6)$ \\
\hline $\mathrm{F}(17)-\mathrm{C}(78)-\mathrm{C}(75)$ & $113.2(7)$ \\
\hline$C(75)-C(76)-C(71)$ & $121.80(19)$ \\
\hline$C(52)-C(51)-C(56)$ & $115.56(18)$ \\
\hline $\mathrm{C}(52)-\mathrm{C}(51)-\mathrm{B}(1)$ & $121.84(17)$ \\
\hline $\mathrm{C}(56)-\mathrm{C}(51)-\mathrm{B}(1)$ & $122.31(17)$ \\
\hline$C(53)-C(52)-C(51)$ & $122.2(2)$ \\
\hline$C(54)-C(53)-C(52)$ & $121.1(2)$ \\
\hline$C(54)-C(53)-C(57)$ & $119.6(2)$ \\
\hline$C(52)-C(53)-C(57)$ & $119.4(2)$ \\
\hline $\mathrm{F}(2)-\mathrm{C}(57)-\mathrm{F}(3 \mathrm{~A})$ & $116.9(9)$ \\
\hline $\mathrm{F}(2)-\mathrm{C}(57)-\mathrm{F}(1)$ & $113.6(8)$ \\
\hline $\mathrm{F}(3 \mathrm{~A})-\mathrm{C}(57)-\mathrm{F}(1)$ & $74.1(7)$ \\
\hline $\mathrm{F}(2)-\mathrm{C}(57)-\mathrm{F}(2 \mathrm{~A})$ & $18.4(10)$ \\
\hline $\mathrm{F}(3 \mathrm{~A})-\mathrm{C}(57)-\mathrm{F}(2 \mathrm{~A})$ & $108.7(6)$ \\
\hline $\mathrm{F}(1)-\mathrm{C}(57)-\mathrm{F}(2 \mathrm{~A})$ & $129.2(7)$ \\
\hline$F(2)-C(57)-F(1 A)$ & $83.2(8)$ \\
\hline $\mathrm{F}(3 \mathrm{~A})-\mathrm{C}(57)-\mathrm{F}(1 \mathrm{~A})$ & $108.1(4)$ \\
\hline $\mathrm{F}(1)-\mathrm{C}(57)-\mathrm{F}(1 \mathrm{~A})$ & $38.0(7)$ \\
\hline $\mathrm{F}(2 \mathrm{~A})-\mathrm{C}(57)-\mathrm{F}(1 \mathrm{~A})$ & $101.5(5)$ \\
\hline $\mathrm{F}(2)-\mathrm{C}(57)-\mathrm{F}(3)$ & 102.1(9) \\
\hline
\end{tabular}




$\begin{array}{lc}\mathrm{F}(3 \mathrm{~A})-\mathrm{C}(57)-\mathrm{F}(3) & 26.3(4) \\ \mathrm{F}(1)-\mathrm{C}(57)-\mathrm{F}(3) & 100.0(7) \\ \mathrm{F}(2 \mathrm{~A})-\mathrm{C}(57)-\mathrm{F}(3) & 88.8(7) \\ \mathrm{F}(1 \mathrm{~A})-\mathrm{C}(57)-\mathrm{F}(3) & 130.5(4) \\ \mathrm{F}(2)-\mathrm{C}(57)-\mathrm{C}(53) & 116.9(7) \\ \mathrm{F}(3 \mathrm{~A})-\mathrm{C}(57)-\mathrm{C}(53) & 115.2(4) \\ \mathrm{F}(1)-\mathrm{C}(57)-\mathrm{C}(53) & 113.0(4) \\ \mathrm{F}(2 \mathrm{~A})-\mathrm{C}(57)-\mathrm{C}(53) & 110.8(4) \\ \mathrm{F}(1 \mathrm{~A})-\mathrm{C}(57)-\mathrm{C}(53) & 111.7(3) \\ \mathrm{F}(3)-\mathrm{C}(57)-\mathrm{C}(53) & 109.2(4) \\ \mathrm{C}(55)-\mathrm{C}(54)-\mathrm{C}(53) & 117.96(19) \\ \mathrm{C}(54)-\mathrm{C}(55)-\mathrm{C}(56) & 121.0(2) \\ \mathrm{C}(54)-\mathrm{C}(55)-\mathrm{C}(58) & 118.85(19) \\ \mathrm{C}(56)-\mathrm{C}(55)-\mathrm{C}(58) & 120.05(19) \\ \mathrm{F}(5)-\mathrm{C}(58)-\mathrm{F}(6) & 106.48(19) \\ \mathrm{F}(5)-\mathrm{C}(58)-\mathrm{F}(4) & 106.15(19) \\ \mathrm{F}(6)-\mathrm{C}(58)-\mathrm{F}(4) & 105.69(19) \\ \mathrm{F}(5)-\mathrm{C}(58)-\mathrm{C}(55) & 113.12(18) \\ \mathrm{F}(6)-\mathrm{C}(58)-\mathrm{C}(55) & 111.49(19) \\ \mathrm{F}(4)-\mathrm{C}(58)-\mathrm{C}(55) & 113.35(18) \\ \mathrm{C}(55)-\mathrm{C}(56)-\mathrm{C}(51) & 122.15(19) \\ \mathrm{C}(82)-\mathrm{C}(81)-\mathrm{C}(86) & 115.83(17) \\ \mathrm{C}(82)-\mathrm{C}(81)-\mathrm{B}(1) & 121.39(16) \\ \mathrm{C}(86)-\mathrm{C}(81)-\mathrm{B}(1) & 122.54(16) \\ \mathrm{C}(83)-\mathrm{C}(82)-\mathrm{C}(81) & 122.35(18) \\ \mathrm{C}(82)-\mathrm{C}(83)-\mathrm{C}(84) & 121.24(18) \\ \mathrm{C}(82)-\mathrm{C}(83)-\mathrm{C}(87) & 118.76(18) \\ \mathrm{C}(84)-\mathrm{C}(83)-\mathrm{C}(87) & 119.94(18) \\ \mathrm{F}(20)-\mathrm{C}(87)-\mathrm{F}(19) & 107.05(19) \\ \mathrm{F}(20)-\mathrm{C}(87)-\mathrm{F}(21) & 105.92(18) \\ \mathrm{F}(19)-\mathrm{C}(87)-\mathrm{F}(21) & 106.22(19) \\ \mathrm{F}(20)-\mathrm{C}(87)-\mathrm{C}(83) & 113.21(18) \\ \mathrm{F}(19)-\mathrm{C}(87)-\mathrm{C}(83) & 112.29(18) \\ \mathrm{C}(83)-\mathrm{C}(84)-\mathrm{C}(85) & 117.34(18) \\ \mathrm{C}(84)-\mathrm{C}(85)-\mathrm{C}(86) & 121.58(18) \\ & \end{array}$




$\begin{array}{ll}\mathrm{C}(84)-\mathrm{C}(85)-\mathrm{C}(88) & 119.48(18) \\ \mathrm{C}(86)-\mathrm{C}(85)-\mathrm{C}(88) & 118.93(18) \\ \mathrm{F}(23)-\mathrm{C}(88)-\mathrm{F}(24) & 106.62(18) \\ \mathrm{F}(23)-\mathrm{C}(88)-\mathrm{F}(22) & 105.98(17) \\ \mathrm{F}(24)-\mathrm{C}(88)-\mathrm{F}(22) & 106.37(17) \\ \mathrm{F}(23)-\mathrm{C}(88)-\mathrm{C}(85) & 112.93(18) \\ \mathrm{F}(24)-\mathrm{C}(88)-\mathrm{C}(85) & 112.31(17) \\ \mathrm{F}(22)-\mathrm{C}(88)-\mathrm{C}(85) & 112.14(18) \\ \mathrm{C}(85)-\mathrm{C}(86)-\mathrm{C}(81) & 121.65(18) \\ \mathrm{Cl}(2 \mathrm{~S})-\mathrm{C}(1 \mathrm{~S})-\mathrm{Cl}(1 \mathrm{~S}) & 129.5(7) \\ \mathrm{Cl}(1 \mathrm{R})-\mathrm{C}(1 \mathrm{R})-\mathrm{Cl}(2 \mathrm{R}) & 133.7(8)\end{array}$


Anisotropic displacement parameters $\left(\AA^{2} \times 10^{3}\right)$ for $4 \mathbf{a}(\mathbf{0 6 1 9 2})$. The anisotropic displacement factor exponent takes the form: $-2 \mathrm{p}^{2}\left[h^{2} \mathrm{a}^{* 2} \mathrm{U}^{11}+\ldots+2 h k \mathrm{a}^{*} \mathrm{~b}^{*} \mathrm{U}^{12}\right]$.

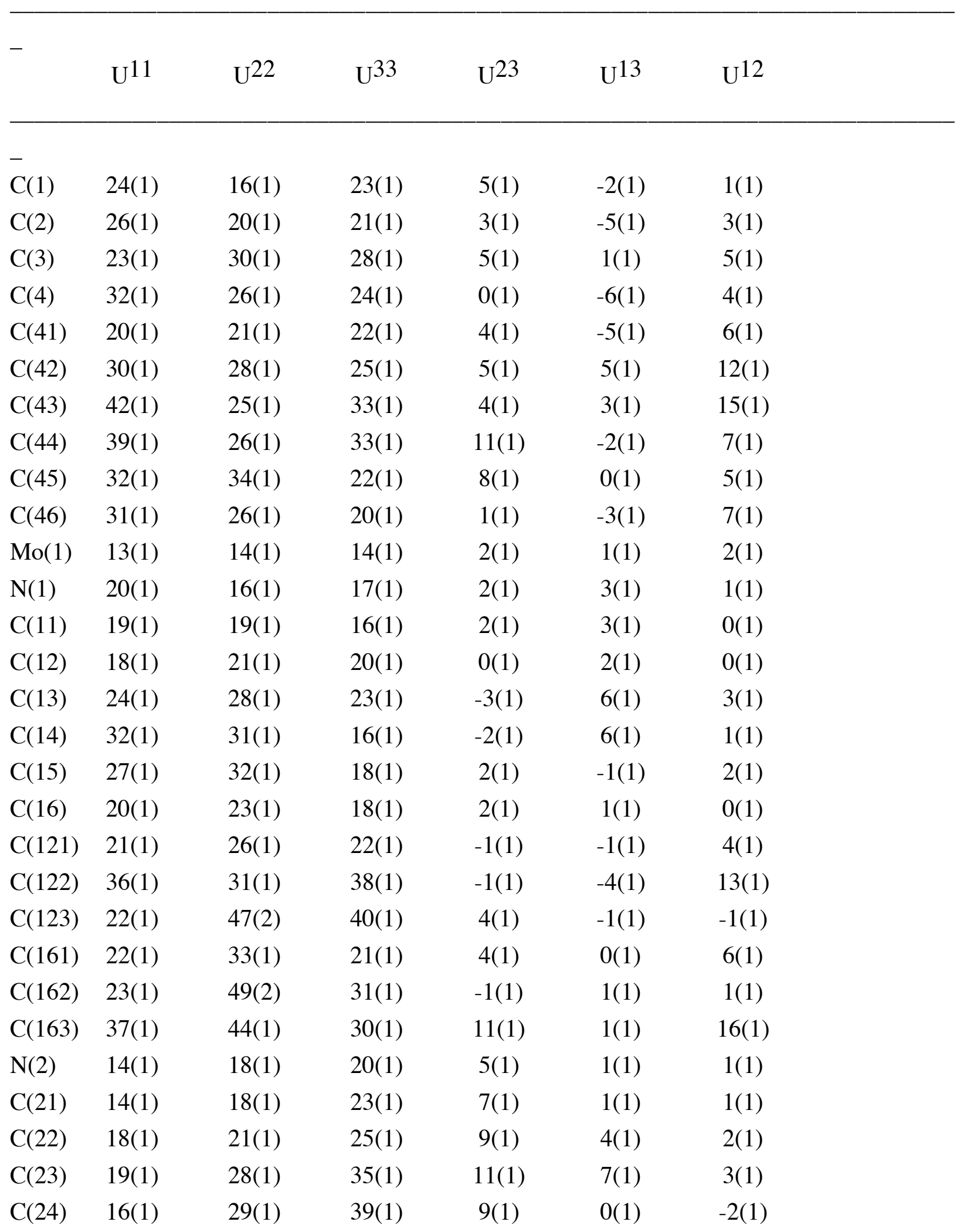




\begin{tabular}{|c|c|c|c|c|c|c|}
\hline$C(25)$ & $23(1)$ & $23(1)$ & $31(1)$ & $4(1)$ & $-3(1)$ & $-2(1)$ \\
\hline$C(26)$ & $20(1)$ & $18(1)$ & $24(1)$ & $5(1)$ & $2(1)$ & $2(1)$ \\
\hline$C(27)$ & $28(1)$ & $30(1)$ & $24(1)$ & $6(1)$ & $7(1)$ & $2(1)$ \\
\hline $\mathrm{C}(28)$ & $28(1)$ & $20(1)$ & $27(1)$ & $0(1)$ & $7(1)$ & $0(1)$ \\
\hline$C(5)$ & $18(1)$ & $18(1)$ & $24(1)$ & $7(1)$ & $2(1)$ & $2(1)$ \\
\hline$C(6)$ & $21(1)$ & $23(1)$ & $41(1)$ & $18(1)$ & $0(1)$ & $1(1)$ \\
\hline$C(7)$ & $17(1)$ & $22(1)$ & $28(1)$ & $10(1)$ & $-1(1)$ & $3(1)$ \\
\hline $\mathrm{C}(8)$ & $15(1)$ & $20(1)$ & $24(1)$ & $5(1)$ & $0(1)$ & $4(1)$ \\
\hline $\mathrm{C}(9)$ & $16(1)$ & $34(1)$ & $46(1)$ & $20(1)$ & $4(1)$ & $7(1)$ \\
\hline $\mathrm{N}(3)$ & $15(1)$ & $16(1)$ & $19(1)$ & $5(1)$ & $3(1)$ & $2(1)$ \\
\hline$C(31)$ & $14(1)$ & $17(1)$ & $22(1)$ & $5(1)$ & $4(1)$ & $3(1)$ \\
\hline$C(32)$ & $17(1)$ & $18(1)$ & $26(1)$ & $2(1)$ & $3(1)$ & $2(1)$ \\
\hline$C(33)$ & $21(1)$ & $20(1)$ & $36(1)$ & $6(1)$ & $6(1)$ & $-1(1)$ \\
\hline$C(34)$ & $26(1)$ & $26(1)$ & $33(1)$ & $10(1)$ & $12(1)$ & $2(1)$ \\
\hline$C(35)$ & $27(1)$ & $26(1)$ & $24(1)$ & $5(1)$ & $10(1)$ & $6(1)$ \\
\hline$C(36)$ & $20(1)$ & $16(1)$ & $23(1)$ & $3(1)$ & $4(1)$ & $4(1)$ \\
\hline$C(37)$ & $21(1)$ & $29(1)$ & $29(1)$ & $-5(1)$ & $0(1)$ & $0(1)$ \\
\hline $\mathrm{C}(38)$ & $29(1)$ & $19(1)$ & $27(1)$ & $-1(1)$ & $6(1)$ & $0(1)$ \\
\hline $\mathrm{O}(1 \mathrm{~T})$ & $20(1)$ & $16(1)$ & $18(1)$ & $3(1)$ & $3(1)$ & $3(1)$ \\
\hline $\mathrm{C}(1 \mathrm{~T})$ & $25(1)$ & $22(1)$ & $19(1)$ & $8(1)$ & $3(1)$ & $4(1)$ \\
\hline $\mathrm{C}(2 \mathrm{~T})$ & $41(1)$ & $21(1)$ & $37(1)$ & $10(1)$ & $15(1)$ & $6(1)$ \\
\hline $\mathrm{C}(3 \mathrm{~T})$ & $56(2)$ & $20(1)$ & $39(1)$ & $6(1)$ & $13(1)$ & $-2(1)$ \\
\hline $\mathrm{C}(4 \mathrm{~T})$ & $30(1)$ & $16(1)$ & $28(1)$ & $3(1)$ & $8(1)$ & $5(1)$ \\
\hline $\mathrm{B}(1)$ & $16(1)$ & $17(1)$ & $16(1)$ & $4(1)$ & $2(1)$ & $2(1)$ \\
\hline$C(61)$ & $17(1)$ & $19(1)$ & $16(1)$ & $4(1)$ & $4(1)$ & $4(1)$ \\
\hline$C(62)$ & $19(1)$ & $22(1)$ & $24(1)$ & $7(1)$ & $1(1)$ & $2(1)$ \\
\hline$C(63)$ & $23(1)$ & $25(1)$ & $30(1)$ & $12(1)$ & $0(1)$ & $4(1)$ \\
\hline$C(67)$ & $35(1)$ & $36(1)$ & $67(2)$ & $29(1)$ & $-9(1)$ & $2(1)$ \\
\hline $\mathrm{F}(7)$ & $86(3)$ & $56(3)$ & $52(2)$ & $37(2)$ & $14(2)$ & $21(2)$ \\
\hline $\mathrm{F}(8)$ & $74(3)$ & $33(2)$ & $86(4)$ & $36(2)$ & $-52(3)$ & $-26(2)$ \\
\hline $\mathrm{F}(9)$ & $65(3)$ & $30(2)$ & $79(3)$ & $19(2)$ & $42(3)$ & $30(2)$ \\
\hline $\mathrm{F}(7 \mathrm{~A})$ & $97(6)$ & $54(4)$ & 134(7) & $62(4)$ & $87(5)$ & $42(4)$ \\
\hline $\mathrm{F}(8 \mathrm{~A})$ & $97(4)$ & $19(2)$ & $71(4)$ & $5(2)$ & $-47(3)$ & $8(2)$ \\
\hline $\mathrm{F}(9 \mathrm{~A})$ & $44(3)$ & $37(4)$ & $156(7)$ & $37(4)$ & $-54(4)$ & $-1(3)$ \\
\hline$C(64)$ & $20(1)$ & $30(1)$ & $24(1)$ & $8(1)$ & $0(1)$ & $7(1)$ \\
\hline$C(65)$ & $18(1)$ & $24(1)$ & $18(1)$ & $0(1)$ & $2(1)$ & $4(1)$ \\
\hline
\end{tabular}




\begin{tabular}{|c|c|c|c|c|c|c|}
\hline$C(68)$ & $24(1)$ & $29(1)$ & $25(1)$ & $-2(1)$ & $-4(1)$ & $5(1)$ \\
\hline $\mathrm{F}(10)$ & $38(4)$ & $31(5)$ & $39(6)$ & $-9(4)$ & $-1(4)$ & $11(3)$ \\
\hline $\mathrm{F}(11)$ & $45(8)$ & $44(8)$ & $34(4)$ & $2(4)$ & $3(4)$ & $-16(5)$ \\
\hline $\mathrm{F}(12)$ & $27(4)$ & $34(4)$ & $40(7)$ & $-8(4)$ & $-16(4)$ & $8(3)$ \\
\hline$F(10 A)$ & $41(3)$ & $28(3)$ & $52(6)$ & $-13(3)$ & $-12(3)$ & $11(2)$ \\
\hline $\mathrm{F}(11 \mathrm{~A})$ & $29(3)$ & $43(5)$ & $43(3)$ & $-3(2)$ & $2(2)$ & $-15(3)$ \\
\hline $\mathrm{F}(12 \mathrm{~A})$ & $44(5)$ & $43(3)$ & $33(3)$ & $-3(3)$ & $-20(4)$ & $9(3)$ \\
\hline$C(66)$ & 19(1) & $20(1)$ & $18(1)$ & $3(1)$ & $2(1)$ & $4(1)$ \\
\hline$C(71)$ & $20(1)$ & $18(1)$ & $14(1)$ & $1(1)$ & $1(1)$ & $2(1)$ \\
\hline$C(72)$ & $23(1)$ & $22(1)$ & $19(1)$ & $4(1)$ & $3(1)$ & $5(1)$ \\
\hline$C(73)$ & $33(1)$ & $22(1)$ & $19(1)$ & $5(1)$ & $1(1)$ & $8(1)$ \\
\hline $\mathrm{C}(77)$ & $40(1)$ & $33(1)$ & $32(1)$ & $15(1)$ & $2(1)$ & $13(1)$ \\
\hline $\mathrm{F}(13)$ & $54(2)$ & 100(3) & $88(3)$ & $79(2)$ & $6(2)$ & $20(2)$ \\
\hline $\mathrm{F}(14)$ & $49(2)$ & $49(2)$ & 113(3) & $32(2)$ & $-40(2)$ & $2(1)$ \\
\hline $\mathrm{F}(15)$ & $142(4)$ & $137(4)$ & $58(2)$ & $-1(2)$ & $-5(2)$ & $123(4)$ \\
\hline$F(13 A)$ & $51(5)$ & $29(4)$ & 121(10) & $31(5)$ & $-16(6)$ & $6(3)$ \\
\hline$F(14 A)$ & 122(9) & 112(9) & $42(5)$ & $-20(5)$ & $-34(5)$ & $95(8)$ \\
\hline $\mathrm{F}(15 \mathrm{~A})$ & $34(4)$ & $92(8)$ & $102(8)$ & $75(7)$ & $19(4)$ & $34(4)$ \\
\hline$C(74)$ & $37(1)$ & $22(1)$ & $20(1)$ & $6(1)$ & $5(1)$ & $1(1)$ \\
\hline$C(75)$ & $26(1)$ & $23(1)$ & $18(1)$ & $3(1)$ & $5(1)$ & $-2(1)$ \\
\hline $\mathrm{C}(78)$ & $30(1)$ & $35(1)$ & $27(1)$ & $9(1)$ & $8(1)$ & $-6(1)$ \\
\hline $\mathrm{F}(16)$ & $36(4)$ & $55(3)$ & $35(3)$ & $4(3)$ & $17(3)$ & $3(2)$ \\
\hline $\mathrm{F}(17)$ & $20(2)$ & $43(4)$ & $35(3)$ & $13(2)$ & $1(2)$ & $-2(3)$ \\
\hline $\mathrm{F}(18)$ & $31(3)$ & $38(3)$ & $54(5)$ & $26(3)$ & $1(3)$ & $-11(2)$ \\
\hline $\mathrm{F}(16 \mathrm{~A})$ & $28(4)$ & $65(5)$ & $49(7)$ & $-9(5)$ & $18(5)$ & $-1(3)$ \\
\hline $\mathrm{F}(17 \mathrm{~A})$ & $30(4)$ & $56(8)$ & $37(3)$ & $4(4)$ & $4(2)$ & $-9(4)$ \\
\hline $\mathrm{F}(18 \mathrm{~A})$ & $50(6)$ & $62(5)$ & $51(7)$ & $25(5)$ & $13(5)$ & $-22(4)$ \\
\hline$C(76)$ & 21(1) & $20(1)$ & $16(1)$ & $2(1)$ & $3(1)$ & $2(1)$ \\
\hline$C(51)$ & $20(1)$ & $18(1)$ & $17(1)$ & $4(1)$ & $3(1)$ & $2(1)$ \\
\hline$C(52)$ & 21(1) & $23(1)$ & $23(1)$ & $2(1)$ & $1(1)$ & $4(1)$ \\
\hline$C(53)$ & $25(1)$ & $27(1)$ & $25(1)$ & $1(1)$ & $-3(1)$ & $0(1)$ \\
\hline$C(57)$ & $29(1)$ & $42(1)$ & $39(1)$ & $-9(1)$ & $-9(1)$ & $4(1)$ \\
\hline $\mathrm{F}(1)$ & $55(5)$ & $65(5)$ & $115(9)$ & $-56(5)$ & $-46(5)$ & $16(4)$ \\
\hline $\mathrm{F}(2)$ & $35(4)$ & 118(8) & $60(7)$ & $53(7)$ & $-21(4)$ & $-10(6)$ \\
\hline $\mathrm{F}(3)$ & $18(2)$ & $81(7)$ & $58(4)$ & $2(4)$ & $-7(2)$ & $-12(3)$ \\
\hline $\mathrm{F}(1 \mathrm{~A})$ & $45(2)$ & $102(4)$ & $45(2)$ & $-37(2)$ & $-21(2)$ & $20(2)$ \\
\hline
\end{tabular}




$\begin{array}{lcccccc}\mathrm{F}(2 \mathrm{~A}) & 51(3) & 59(2) & 63(4) & 8(2) & -35(3) & 11(2) \\ \mathrm{F}(3 \mathrm{~A}) & 27(2) & 90(5) & 65(3) & 23(3) & -4(2) & -15(2) \\ \mathrm{C}(54) & 34(1) & 22(1) & 22(1) & 0(1) & 0(1) & 1(1) \\ \mathrm{C}(55) & 32(1) & 20(1) & 20(1) & 5(1) & 5(1) & 6(1) \\ \mathrm{C}(58) & 40(1) & 25(1) & 22(1) & 1(1) & 3(1) & 12(1) \\ \mathrm{F}(4) & 34(1) & 41(1) & 48(1) & -4(1) & 4(1) & 17(1) \\ \mathrm{F}(5) & 64(1) & 51(1) & 25(1) & -5(1) & 2(1) & 31(1) \\ \mathrm{F}(6) & 55(1) & 34(1) & 51(1) & 19(1) & 13(1) & 20(1) \\ \mathrm{C}(56) & 23(1) & 22(1) & 18(1) & 4(1) & 2(1) & 4(1) \\ \mathrm{C}(81) & 17(1) & 18(1) & 16(1) & 4(1) & 1(1) & 3(1) \\ \mathrm{C}(82) & 19(1) & 18(1) & 19(1) & 5(1) & 2(1) & 2(1) \\ \mathrm{C}(83) & 25(1) & 17(1) & 19(1) & 2(1) & 2(1) & 6(1) \\ \mathrm{C}(87) & 33(1) & 20(1) & 26(1) & 2(1) & 4(1) & 6(1) \\ \mathrm{F}(19) & 42(1) & 28(1) & 65(1) & -16(1) & -12(1) & 4(1) \\ \mathrm{F}(20) & 60(1) & 28(1) & 43(1) & 2(1) & 23(1) & 18(1) \\ \mathrm{F}(21) & 67(1) & 22(1) & 34(1) & 8(1) & 5(1) & 13(1) \\ \mathrm{C}(84) & 23(1) & 24(1) & 21(1) & 4(1) & 6(1) & 7(1) \\ \mathrm{C}(85) & 19(1) & 20(1) & 21(1) & 6(1) & 5(1) & 3(1) \\ \mathrm{C}(88) & 25(1) & 24(1) & 29(1) & 5(1) & 11(1) & 4(1) \\ \mathrm{F}(22) & 37(1) & 29(1) & 34(1) & 15(1) & 11(1) & 3(1) \\ \mathrm{F}(23) & 27(1) & 36(1) & 59(1) & 11(1) & 24(1) & 7(1) \\ \mathrm{F}(24) & 35(1) & 30(1) & 36(1) & 2(1) & 7(1) & -10(1) \\ \mathrm{C}(86) & 19(1) & 17(1) & 19(1) & 3(1) & 3(1) & 3(1) \\ \mathrm{C}(1 \mathrm{~S}) & 64(5) & 198(12) & 69(6) & 25(7) & -25(4) & 5(6) \\ \mathrm{Cl}(1 \mathrm{~S}) & 51(1) & 72(1) & 79(2) & 6(1) & -1(1) & 16(1) \\ \mathrm{Cl}(2 \mathrm{~S}) & 55(1) & 105(2) & 117(3) & 34(2) & -1(2) & 30(1) \\ \mathrm{C}(1 \mathrm{R}) & 69(6) & 167(11) & 71(7) & -34(7) & 26(5) & 8(7) \\ \mathrm{Cl}(1 \mathrm{R}) & 72(2) & 130(2) & 83(3) & 22(2) & 20(1) & 56(2) \\ \mathrm{Cl}(2 \mathrm{R}) & 47(1) & 78(2) & 122(3) & 14(2) & -3(2) & 26(1)\end{array}$


Hydrogen coordinates $\left(\times 10^{4}\right)$ and isotropic displacement parameters $\left(\AA^{2} \times 10^{3}\right)$ for 4a (06192).

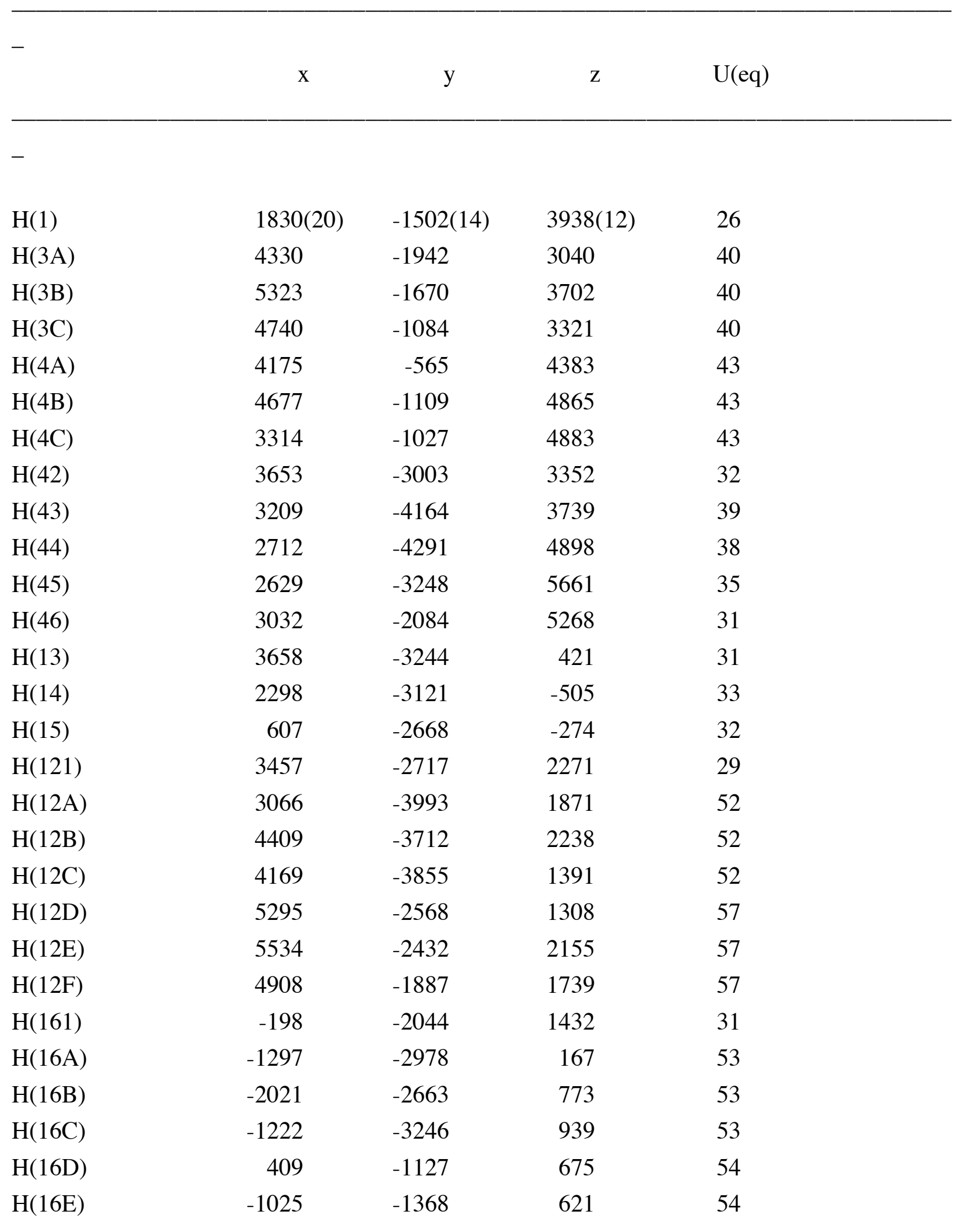




\begin{tabular}{|c|c|c|c|c|}
\hline $\mathrm{H}(16 \mathrm{~F})$ & -303 & -1669 & 6 & 54 \\
\hline $\mathrm{H}(23)$ & 5521 & -109 & 1857 & 32 \\
\hline $\mathrm{H}(24)$ & 6270 & 581 & 2942 & 35 \\
\hline $\mathrm{H}(25)$ & 4971 & 756 & 3807 & 32 \\
\hline $\mathrm{H}(27 \mathrm{~A})$ & 3445 & -428 & 1004 & 41 \\
\hline $\mathrm{H}(27 \mathrm{~B})$ & 2463 & -1005 & 1337 & 41 \\
\hline $\mathrm{H}(27 \mathrm{C})$ & 3762 & -1172 & 1232 & 41 \\
\hline $\mathrm{H}(28 \mathrm{~A})$ & 3063 & 556 & 4233 & 39 \\
\hline $\mathrm{H}(28 \mathrm{~B})$ & 2169 & -210 & 3939 & 39 \\
\hline $\mathrm{H}(28 \mathrm{C})$ & 2027 & 527 & 3613 & 39 \\
\hline $\mathrm{H}(6 \mathrm{~A})$ & 2005 & 760 & 2310 & 42 \\
\hline $\mathrm{H}(6 \mathrm{~B})$ & 884 & 604 & 1721 & 42 \\
\hline $\mathrm{H}(6 \mathrm{C})$ & 2069 & 314 & 1555 & 42 \\
\hline $\mathrm{H}(7)$ & -649 & -288 & 1838 & 27 \\
\hline $\mathrm{H}(9 \mathrm{~A})$ & -2728 & -1508 & 2389 & 46 \\
\hline $\mathrm{H}(9 \mathrm{~B})$ & -2436 & -691 & 2168 & 46 \\
\hline $\mathrm{H}(9 \mathrm{C})$ & -2402 & -816 & 2988 & 46 \\
\hline $\mathrm{H}(33)$ & -3440 & -3335 & 3414 & 31 \\
\hline $\mathrm{H}(34)$ & -3426 & -2789 & 4572 & 33 \\
\hline $\mathrm{H}(35)$ & -2136 & -1658 & 4960 & 30 \\
\hline $\mathrm{H}(37 \mathrm{~A})$ & -2495 & -2627 & 2034 & 42 \\
\hline $\mathrm{H}(37 \mathrm{~B})$ & -2656 & -3415 & 2319 & 42 \\
\hline $\mathrm{H}(37 \mathrm{C})$ & -1339 & -2944 & 2249 & 42 \\
\hline $\mathrm{H}(38 \mathrm{~A})$ & -766 & -601 & 4751 & 39 \\
\hline $\mathrm{H}(38 \mathrm{~B})$ & -687 & -442 & 3942 & 39 \\
\hline $\mathrm{H}(38 \mathrm{C})$ & 293 & -812 & 4321 & 39 \\
\hline $\mathrm{H}(1 \mathrm{~T} 1)$ & -218 & -2771 & 4064 & 26 \\
\hline $\mathrm{H}(1 \mathrm{~T} 2)$ & 1043 & -3029 & 4061 & 26 \\
\hline $\mathrm{H}(2 \mathrm{~T} 1)$ & -196 & -4145 & 4095 & 39 \\
\hline $\mathrm{H}(2 \mathrm{~T} 2)$ & -1304 & -3921 & 3664 & 39 \\
\hline $\mathrm{H}(3 \mathrm{~T} 1)$ & -758 & -4498 & 2701 & 47 \\
\hline $\mathrm{H}(3 \mathrm{~T} 2)$ & 521 & -4524 & 3098 & 47 \\
\hline $\mathrm{H}(4 \mathrm{~T} 1)$ & 1419 & -3532 & 2637 & 29 \\
\hline $\mathrm{H}(4 \mathrm{~T} 2)$ & 184 & -3579 & 2156 & 29 \\
\hline $\mathrm{H}(62)$ & 2247 & -1868 & -2948 & 26 \\
\hline $\mathrm{H}(64)$ & -632 & -2664 & -4390 & 29 \\
\hline
\end{tabular}




\begin{tabular}{lrrrr}
$\mathrm{H}(66)$ & 670 & -4029 & -3161 & 23 \\
$\mathrm{H}(72)$ & 3794 & -4009 & -1633 & 25 \\
$\mathrm{H}(74)$ & 1189 & -5240 & -651 & 32 \\
$\mathrm{H}(76)$ & 371 & -3765 & -1927 & 23 \\
$\mathrm{H}(52)$ & 4792 & -2585 & -1492 & 27 \\
$\mathrm{H}(54)$ & 4226 & -793 & -291 & 32 \\
$\mathrm{H}(56)$ & 1632 & -1957 & -1777 & 25 \\
$\mathrm{H}(82)$ & 3059 & -4412 & -2994 & 23 \\
$\mathrm{H}(84)$ & 5977 & -3770 & -4060 & 26 \\
$\mathrm{H}(86)$ & 4649 & -2257 & -2855 & 22 \\
$\mathrm{H}(1 \mathrm{~S} 1)$ & -2761 & -2852 & -2403 & 137 \\
$\mathrm{H}(1 \mathrm{~S} 2)$ & -2081 & -3507 & -2417 & 137 \\
$\mathrm{H}(1 \mathrm{R} 1)$ & -2937 & -2495 & -1844 & 129 \\
$\mathrm{H}(1 \mathrm{R} 2)$ & -2022 & -2487 & -1183 & 129 \\
& & & & \\
\hline
\end{tabular}

